\title{
Asymmetric Total Syntheses of (+)-Davisinol and \\ (+)-18-Benzoyldavisinol: A HAT-Initiated Transannular Redox \\ Radical Approach
}

\author{
Kuan Yu,${ }^{\dagger}$ Fengjie Yao, ${ }^{\dagger}$ Qingrui Zeng,${ }^{\dagger}$ Hujun Xie,${ }^{\S}$ and Hanfeng Ding $*, \dagger, \dagger$ \\ 'Department of Chemistry, Zhejiang University, Hangzhou 310058, China \\ ${ }^{+}$State Key Laboratory of Elemento-Organic Chemistry, Nankai University, Tianjin 300071, \\ China \\ ${ }^{\S}$ Department of Applied Chemistry, Zhejiang Gongshang University, Hangzhou 310018, China \\ E-mail: hfding@zju.edu.cn
}

\section{Supporting Information Available}

I) Experimental Procedures and Spectroscopic Data of Compounds

II) Computational Details and Coordinates of Computed Stationary Points

III) Abbreviations

IV) References

V) ${ }^{1} \mathrm{H}$ and ${ }^{13} \mathrm{C}$ NMR Spectra of Compounds

VI) HPLC Chromatographs 


\section{I) Experimental Procedures and Spectroscopic Data of Compounds}

General Procedures. All reactions were carried out under an argon atmosphere with dry solvents under anhydrous conditions, unless otherwise noted. Anhydrous methylene chloride $\left(\mathrm{CH}_{2} \mathrm{Cl}_{2}\right)$, 1,2-dichloroethane (DCE) and 1,4-dioxane were distilled before use from calcium hydride. Anhydrous benzene, diethyl ether $\left(\mathrm{Et}_{2} \mathrm{O}\right)$, tetrahydrofuran (THF) and toluene were distilled before use from sodium-benzophenone ketyl. Acetic acid $(\mathrm{AcOH})$, acetone, chloroform $\left(\mathrm{CHCl}_{3}\right)$, diisopropylamine $\left(i-\mathrm{Pr}_{2} \mathrm{NH}\right)$, ethanol $(\mathrm{EtOH})$, ethyl acetate (EtOAc), 1,1,1,3,3,3-hexafluoro2-propanol (HFIP), isopropanol ( $i$-PrOH), methanol (MeOH), $N, N^{\prime}$-diisopropylethylamine $\left(i-\mathrm{Pr}_{2} \mathrm{NEt}\right), N, N^{\prime}$-dimethylformamide $(\mathrm{DMF})$, triethylamine $\left(\mathrm{Et}_{3} \mathrm{~N}\right)$, and trifluorotoluene $\left(\mathrm{CF}_{3} \mathrm{Ph}\right)$ were purchased at the highest commercial quality and used without further purification. Yields refer to chromatographically homogeneous materials. Reactions that required heating were operated on a magnetic stirrer with an oil bath. Solvent degassing was conducted by bubbling with a stream of argon for 30-60 min. Reactions were monitored by thin-layer chromatography (TLC) carried out on silica gel plates $(0.4-0.5 \mathrm{~mm})$ using UV light as visualizing agent and an ethanolic solution of ammonium molybdate, anisaldehyde, and heat as developing agents. Flash chromatography was performed with silica gel (200-300 mesh) or basic aluminium oxide (100-200 mesh) under pressure. NMR spectra were recorded on a Bruker AV-400 or a Zhongke-Niujin 400 NMR spectrometer and calibrated using residual undeuterated solvent as an internal reference. The following abbreviations were used to explain the multiplicities: $\mathrm{s}=$ singlet, $\mathrm{d}=$ doublet, $\mathrm{t}=$ triplet, $\mathrm{q}=$ quartet, $\mathrm{br}=$ broad, $\mathrm{dd}=$ doublet of doublets, $\mathrm{ddd}=$ doublet of doublet of doublets, $\mathrm{td}=$ triplet of doublets, $\mathrm{m}=$ multiplet. Melting points (m.p.) are uncorrected, and recorded on a Buchi B-540 melting point apparatus. Optical rotation data were obtained on a PerkinElmer Model 341 Polarimeter. High performance liquid chromatography (HPLC) analyses 
were performed on a Shimadzu LC-20A HPLC system, and the detection of eluent was carried out with a photodiode array detector at $210 \mathrm{~nm}$. High-resolution mass spectra (HRMS) were recorded on Waters MALDI SYNAPT G2-Si High Definition Mass Spectrometry. IR spectra were recorded on a Thermo Scientific Nicolet iS10 spectrometer and are reported in terms of frequency of absorption $\left(\mathrm{cm}^{-1}\right)$.

\section{Transannular reductive radical cyclization of keto-epoxide 9:}

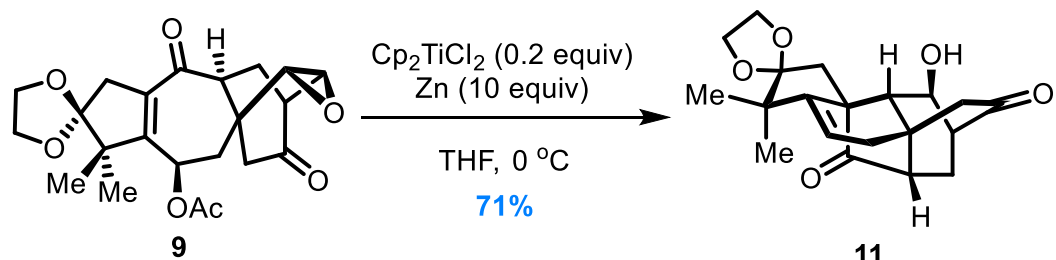

9

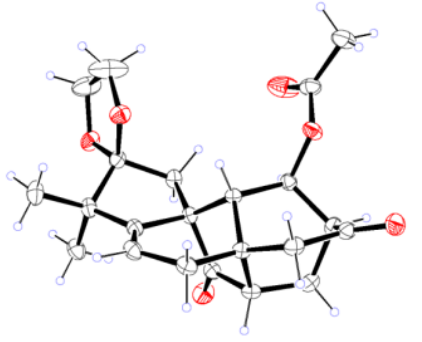

X-ray of 11'

[CCDC 2086697]

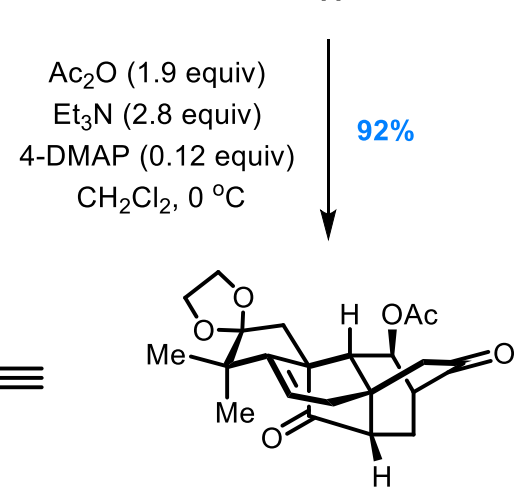

$11^{\prime}$

To a stirred solution of $\mathrm{Cp}_{2} \mathrm{TiCl}_{2}\left(6.0 \mathrm{mg}, 24 \mu \mathrm{mol}, 0.2\right.$ equiv) in $\mathrm{THF}(5 \mathrm{~mL})$ at $0{ }^{\circ} \mathrm{C}$ was added $\mathrm{Zn}$ dust (79 mg, $1.2 \mathrm{mmol}, 10$ equiv). The resulting mixture was stirred at $0{ }^{\circ} \mathrm{C}$ for $0.5 \mathrm{~h}$ before it was added a solution of keto-epoxide 9 (48 mg, $0.12 \mathrm{mmol}, 1.0$ equiv) in THF ( $5 \mathrm{~mL}$ ). The reaction was stirred at $0{ }^{\circ} \mathrm{C}$ for an additional $1.5 \mathrm{~h}$ before it was added $\mathrm{NaHCO}_{3}(5 \mathrm{~mL}$, sat. aq.). The layers were separated, and the aqueous layer was extracted with EtOAc $(3 \times 5 \mathrm{~mL})$. The combined organic layers were washed with brine $(5 \mathrm{~mL})$, dried $\left(\mathrm{Na}_{2} \mathrm{SO}_{4}\right)$ and concentrated in vacuo. Flash column chromatography (silica gel, hexanes:EtOAc 1:1) afforded pentacyclic alcohol 11 (29 mg, 71\%) as a colorless oil, whose structure was characterized by the 
corresponding acetate $\mathbf{1 1}^{\prime}$.

To a stirred solution of pentacyclic alcohol 11 (29 mg, $85 \mu$ mol, 1.0 equiv) in $\mathrm{CH}_{2} \mathrm{Cl}_{2}(5 \mathrm{~mL})$ at $0{ }^{\circ} \mathrm{C}$ were sequentially added $\mathrm{Et}_{3} \mathrm{~N}\left(33 \mu \mathrm{L}, 0.24 \mathrm{mmol}, 2.8\right.$ equiv), $\mathrm{Ac}_{2} \mathrm{O}$ (15 $\mu \mathrm{L}, 0.16 \mathrm{mmol}$, 1.9 equiv) and 4-DMAP ( $1.2 \mathrm{mg}, 10 \mu \mathrm{mol}, 0.12$ equiv). The resulting mixture was stirred at $0{ }^{\circ} \mathrm{C}$ for $0.5 \mathrm{~h}$ before it was added $\mathrm{NaHCO}_{3}(5 \mathrm{~mL}$, sat. aq.). The layers were separated, and the aqueous layer was extracted with $\mathrm{CH}_{2} \mathrm{Cl}_{2}(3 \times 2 \mathrm{~mL})$. The combined organic layers were dried $\left(\mathrm{Na}_{2} \mathrm{SO}_{4}\right)$ and concentrated in vacuo. Flash column chromatography (silica gel, hexanes:EtOAc 2:1) afforded acetate 11' (30 mg, 92\%) as a white solid. 11': CCDC 2086697; m.p. 188-189 ${ }^{\circ} \mathrm{C}$ $\left(\mathrm{CH}_{2} \mathrm{Cl}_{2} /\right.$ hexanes); $R_{\mathrm{f}}=0.30$ (silica gel, hexanes:EtOAc 2:1); ${ }^{1} \mathrm{H}$ NMR $\left(400 \mathrm{MHz}, \mathrm{CDCl}_{3}\right) \delta=$ $5.45(\mathrm{t}, J=3.4 \mathrm{~Hz}, 1 \mathrm{H}), 4.93(\mathrm{~d}, J=4.2 \mathrm{~Hz}, 1 \mathrm{H}), 4.07-3.89(\mathrm{~m}, 4 \mathrm{H}), 2.64-2.50(\mathrm{~m}, 3 \mathrm{H}), 2.41-$ $2.29(\mathrm{~m}, 4 \mathrm{H}), 2.28-2.16(\mathrm{~m}, 2 \mathrm{H}), 2.00(\mathrm{~s}, 3 \mathrm{H}), 1.95(\mathrm{~d}, J=2.9 \mathrm{~Hz}, 1 \mathrm{H}), 1.89$ (dd, $J=15.1,4.7$ $\mathrm{Hz}, 1 \mathrm{H}), 1.08$ (s, $3 \mathrm{H}), 1.01 \mathrm{ppm}(\mathrm{s}, 3 \mathrm{H}) ;{ }^{13} \mathrm{C} \mathrm{NMR}\left(100 \mathrm{MHz}, \mathrm{CDCl}_{3}\right) \delta=212.5,209.3,169.6$, $150.4,118.0,116.3,72.7,66.0,64.4,58.2,51.3,50.0,48.2,45.8,43.0,39.9,38.1,33.5,27.1,23.1$, 21.2, 19.4 ppm; ; IR (film) $v_{\max }=2942,2885,1739,1466,1434,1377,1273,1225,1131,1041$, 1019, 891, $734 \mathrm{~cm}^{-1}$; HRMS (ESI): calcd for $\mathrm{C}_{22} \mathrm{H}_{26} \mathrm{NaO}_{6}{ }^{+}[\mathrm{M}+\mathrm{Na}]^{+} 409.1622$, found 409.1626 . 9: ${ }^{1} R_{\mathrm{f}}=0.40$ (silica gel, hexanes:EtOAc 2:1); ${ }^{1} \mathrm{H}$ NMR $\left(400 \mathrm{MHz}, \mathrm{CDCl}_{3}\right) \delta=5.99-5.85(\mathrm{~m}, 1 \mathrm{H})$, 4.01-3.89 (m, $4 \mathrm{H}), 3.52(\mathrm{t}, J=4.6 \mathrm{~Hz}, 1 \mathrm{H}), 3.00(\mathrm{~d}, J=4.5 \mathrm{~Hz}, 1 \mathrm{H}), 2.98-2.91(\mathrm{~m}, 2 \mathrm{H}), 2.88$ $(\mathrm{dd}, J=15.9,3.3 \mathrm{~Hz}, 1 \mathrm{H}), 2.73(\mathrm{ddd}, J=14.2,4.7,2.8 \mathrm{~Hz}, 1 \mathrm{H}), 2.57(\mathrm{~d}, J=15.9 \mathrm{~Hz}, 1 \mathrm{H}), 2.37$ $(\mathrm{d}, J=18.6 \mathrm{~Hz}, 1 \mathrm{H}), 2.28-2.18(\mathrm{~m}, 3 \mathrm{H}), 2.16(\mathrm{~s}, 3 \mathrm{H}), 1.71(\mathrm{ddd}, \mathrm{J}=13.9,9.6,2.3 \mathrm{~Hz}, 1 \mathrm{H})$, $1.32(\mathrm{~s}, 3 \mathrm{H}), 1.10 \mathrm{ppm}(\mathrm{s}, 3 \mathrm{H}) ;{ }^{13} \mathrm{C} \mathrm{NMR}\left(100 \mathrm{MHz}, \mathrm{CDCl}_{3}\right) \delta=207.2,197.2,169.4,161.9$, $137.4,116.8,68.8,65.6,64.6,55.0,54.2,51.7,49.8,47.2,46.7,44.1,40.7,36.8,22.6,21.6,21.3$, 18.9 ppm; IR (film) $v_{\max }=2969,2873,1736,1659,1480,1367,1232,1210,1068,1035,845,726$ $\mathrm{cm}^{-1}$; HRMS (ESI): calcd for $\mathrm{C}_{22} \mathrm{H}_{26} \mathrm{NaO}_{7}^{+}[\mathrm{M}+\mathrm{Na}]^{+} 425.1571$, found 425.1578 . 


\section{Preparation of diol S-1:}

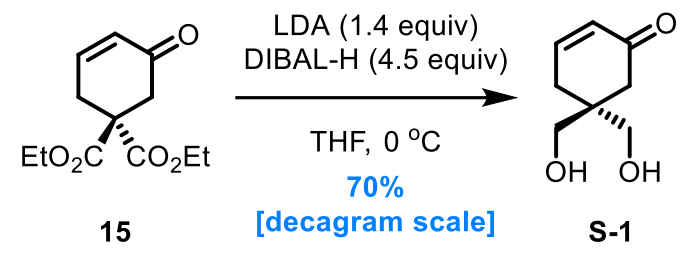

To a stirred solution of $i-\operatorname{Pr}_{2} \mathrm{NH}\left(37.8 \mathrm{~mL}, 270 \mathrm{mmol}, 1.5\right.$ equiv) in THF (200 mL) at $-78{ }^{\circ} \mathrm{C}$ was added $n$-BuLi (100 mL, $2.5 \mathrm{M}$ in hexanes, $250 \mathrm{mmol}, 1.4$ equiv). The resulting mixture was stirred at $-78{ }^{\circ} \mathrm{C}$ for 20 min before it was warmed to $0{ }^{\circ} \mathrm{C}$ and stirred for an additional 15 min. After a solution of the commercially available cyclohexenone $\mathbf{1 5}^{2}$ (43.2 $\mathrm{g}, 180 \mathrm{mmol}, 1.0$ equiv) in THF $(100 \mathrm{~mL})$ was added, the resulting mixture was stirred at $0{ }^{\circ} \mathrm{C}$ for $0.5 \mathrm{~h}$ before it was added DIBAL-H (540 mL, 1.5 $\mathrm{M}$ in toluene, $810 \mathrm{mmol}, 4.5$ equiv). The reaction was stirred for further $1 \mathrm{~h}$ before it was added Rochelle salt (500 mL, sat. aq.). The layers were separated, and the aqueous layer was extracted with $\mathrm{Et}_{2} \mathrm{O}(3 \times 200 \mathrm{~mL})$. The combined organic layers were washed with brine $(200 \mathrm{~mL})$, dried $\left(\mathrm{Na}_{2} \mathrm{SO}_{4}\right)$ and concentrated in vacuo. Flash column chromatography (silica gel, EtOAc) afforded diol S-1 $(19.7 \mathrm{~g}, 70 \%)$ as a colorless oil. S-1: $R_{\mathrm{f}}=$ 0.20 (silica gel, EtOAc); ${ }^{1} \mathrm{H} \mathrm{NMR}\left(400 \mathrm{MHz}, \mathrm{CDCl}_{3}\right) \delta=6.90(\mathrm{dt}, J=10.1,4.1 \mathrm{~Hz}, 1 \mathrm{H}), 6.01(\mathrm{dt}$, $J=10.1,2.0 \mathrm{~Hz}, 1 \mathrm{H}), 3.57$ (s, $4 \mathrm{H}), 3.55$ (brs, $2 \mathrm{H}), 2.38(\mathrm{~s}, 2 \mathrm{H}), 2.36 \mathrm{ppm}(\mathrm{dd}, J=3.7,1.9 \mathrm{~Hz}$, $2 \mathrm{H}) ;{ }^{13} \mathrm{C} \mathrm{NMR}\left(100 \mathrm{MHz}, \mathrm{CDCl}_{3}\right) \delta=199.7,148.9,129.2,66.8$ (2C), 42.6, 41.9, 29.9 ppm; IR (film) $v_{\max }=3404,2966,2878,1667,1392,1258,1092,1038,735 \mathrm{~cm}^{-1}$; HRMS (ESI): calcd for $\mathrm{C}_{8} \mathrm{H}_{12} \mathrm{NaO}_{3}{ }^{+}[\mathrm{M}+\mathrm{Na}]^{+}$179.0679, found 179.0682.

\section{Preparation of acetonide 16:}

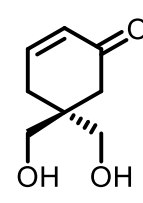

S-1

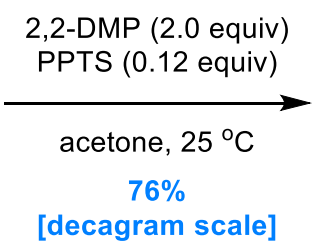

[decagram scale]

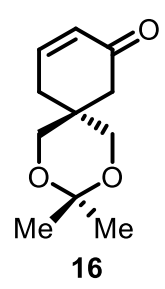

16 
To a stirred solution of diol S-1 (40.6 g, $260 \mathrm{mmol}, 1.0$ equiv) in acetone (300 mL) at $25{ }^{\circ} \mathrm{C}$ were sequentially added 2,2-DMP (63.8 mL, $520 \mathrm{mmol}, 2.0$ equiv) and PPTS (7.54 g, $30.0 \mathrm{mmol}$, 0.12 equiv). The resulting mixture was stirred for $3 \mathrm{~h}$ before it was added $\mathrm{NaHCO}_{3}(150 \mathrm{~mL}$, sat. aq.). The layers were separated, and the aqueous layer was extracted with EtOAc $(3 \times 50 \mathrm{~mL})$. The combined organic layers were washed with brine $(50 \mathrm{~mL})$, dried $\left(\mathrm{Na}_{2} \mathrm{SO}_{4}\right)$ and concentrated in vacuo. Flash column chromatography (silica gel, hexanes:EtOAc 2:1) afforded acetonide 16 $(38.7 \mathrm{~g}, 76 \%)$ as a white amorphous solid. 16: $R_{\mathrm{f}}=0.45$ (silica gel, hexanes:EtOAc $\left.2: 1\right) ;{ }^{1} \mathrm{H}$ NMR $\left(400 \mathrm{MHz}, \mathrm{CDCl}_{3}\right) \delta=6.90(\mathrm{dt}, J=10.6,4.2 \mathrm{~Hz}, 1 \mathrm{H}), 6.04(\mathrm{dt}, J=10.1,2.1 \mathrm{~Hz}, 1 \mathrm{H}), 3.66(\mathrm{~s}, 4$ $\mathrm{H}), 2.58-2.50(\mathrm{~m}, 2 \mathrm{H}), 2.32(\mathrm{~s}, 2 \mathrm{H}), 1.42(\mathrm{~s}, 3 \mathrm{H}), 1.41 \mathrm{ppm}(\mathrm{s}, 3 \mathrm{H}) ;{ }^{13} \mathrm{C} \mathrm{NMR}(100 \mathrm{MHz}$, $\left.\mathrm{CDCl}_{3}\right) \delta=197.4,147.8,129.6,98.6,67.9(2 \mathrm{C}), 43.4,35.6,31.8,26.1,21.4$ ppm; IR (film) $v_{\max }=$ 2939, 2871, 1674, 1455, 1388, 1249, 1210, 1068, 1038, 823, $731 \mathrm{~cm}^{-1}$; HRMS (ESI): calcd for $\mathrm{C}_{11} \mathrm{H}_{16} \mathrm{NaO}_{3}{ }^{+}[\mathrm{M}+\mathrm{Na}]^{+}$219.0992, found 219.0997.

\section{Preparation of aldehyde 17:}

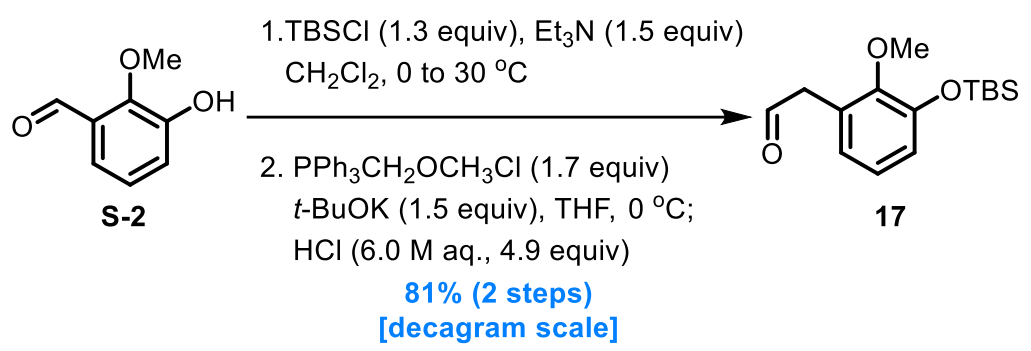

To a stirred solution of the commercially available 3-hydroxy-2-methoxybenzaldehyde (S-2, $77.5 \mathrm{~g}, 510 \mathrm{mmol}, 1.0$ equiv) in $\mathrm{CH}_{2} \mathrm{Cl}_{2}(500 \mathrm{~mL})$ at $0{ }^{\circ} \mathrm{C}$ were sequentially added $\mathrm{Et}_{3} \mathrm{~N}(107 \mathrm{~mL}$, 770 mmol, 1.5 equiv) and $\mathrm{TBSCl}(99.5 \mathrm{~g}, 660 \mathrm{mmol}, 1.3$ equiv). The resulting mixture was warmed to $30{ }^{\circ} \mathrm{C}$ and stirred for $6 \mathrm{~h}$ before it was cooled to $25{ }^{\circ} \mathrm{C}$ and added $\mathrm{NaHCO}_{3}(200 \mathrm{~mL}$, sat. aq.). The layers were separated, and the aqueous layer was extracted with $\mathrm{CH}_{2} \mathrm{Cl}_{2}(3 \times 100$ $\mathrm{mL})$. The combined organic layers were washed with brine $(100 \mathrm{~mL})$, dried $\left(\mathrm{Na}_{2} \mathrm{SO}_{4}\right)$ and 
concentrated in vacuo to afford the crude silyl ether as a pale yellow oil, which was used without further purification.

To a stirred solution of $\mathrm{Ph}_{3} \mathrm{PCH}_{2} \mathrm{OCH}_{3} \mathrm{Cl}(290 \mathrm{~g}, 847 \mathrm{mmol}, 1.7$ equiv) in THF (600 mL) at 0 ${ }^{\circ} \mathrm{C}$ was added $t$-BuOK $\left(86.4 \mathrm{~g}, 770 \mathrm{mmol}, 1.5\right.$ equiv). The resulting mixture was warmed to $25{ }^{\circ} \mathrm{C}$ and stirred for $0.5 \mathrm{~h}$ before it was cooled to $0{ }^{\circ} \mathrm{C}$ and added a solution of the silyl ether (crude, obtained above) in THF (200 mL). The reaction was stirred at $0{ }^{\circ} \mathrm{C}$ for an additional $2 \mathrm{~h}$ before it was added $\mathrm{HCl}(417 \mathrm{~mL}, 6.0 \mathrm{M}$ aq., $2.50 \mathrm{~mol}, 4.9$ equiv). The resulting mixture was stirred at 25 ${ }^{\circ} \mathrm{C}$ for $5 \mathrm{~h}$ before it was extracted with EtOAc $(3 \times 200 \mathrm{~mL})$. The combined organic layers were washed with $\mathrm{NaHCO}_{3}\left(200 \mathrm{~mL}\right.$, sat. aq.) and brine $(200 \mathrm{~mL})$, dried $\left(\mathrm{Na}_{2} \mathrm{SO}_{4}\right)$ and concentrated in vacuo. Flash column chromatography (silica gel, hexanes:EtOAc 20:1) afforded aldehyde 17 (116 g, $81 \%$ over two steps) as a colorless oil. 17: $R_{\mathrm{f}}=0.45$ (silica gel, hexanes:EtOAc 20:1); ${ }^{1} \mathrm{H} \mathrm{NMR}$ $\left(400 \mathrm{MHz}, \mathrm{CDCl}_{3}\right) \delta=9.71(\mathrm{t}, J=2.2 \mathrm{~Hz}, 1 \mathrm{H}), 6.95(\mathrm{t}, J=7.8 \mathrm{~Hz}, 1 \mathrm{H}), 6.83(\mathrm{dd}, J=8.2,1.6$ $\mathrm{Hz}, 1 \mathrm{H}), 6.76(\mathrm{dd}, J=7.6,1.6 \mathrm{~Hz}, 1 \mathrm{H}), 3.76(\mathrm{~s}, 3 \mathrm{H}), 3.66(\mathrm{~d}, J=2.2 \mathrm{~Hz}, 2 \mathrm{H}), 1.02(\mathrm{~s}, 9 \mathrm{H})$, $0.20 \mathrm{ppm}(\mathrm{s}, 6 \mathrm{H}) ;{ }^{13} \mathrm{C} \mathrm{NMR}\left(100 \mathrm{MHz}, \mathrm{CDCl}_{3}\right) \delta=199.9,150.0,149.1,127.0,124.3,123.8$, 121.1, 60.2, 45.5, 25.8 (3C), 18.4, -4.4 ppm (2C); IR (film) $v_{\max }=2931,2858,2716,1727,1587$, 1474, 1288, 1253, 1040, 1008, 839, $782 \mathrm{~cm}^{-1}$; HRMS (ESI): calcd for $\mathrm{C}_{15} \mathrm{H}_{24} \mathrm{NaO}_{3} \mathrm{Si}^{+}[\mathrm{M}+\mathrm{Na}]^{+}$ 303.1387, found 303.1385.

\section{Preparation of homobenzylic alcohol 18:}
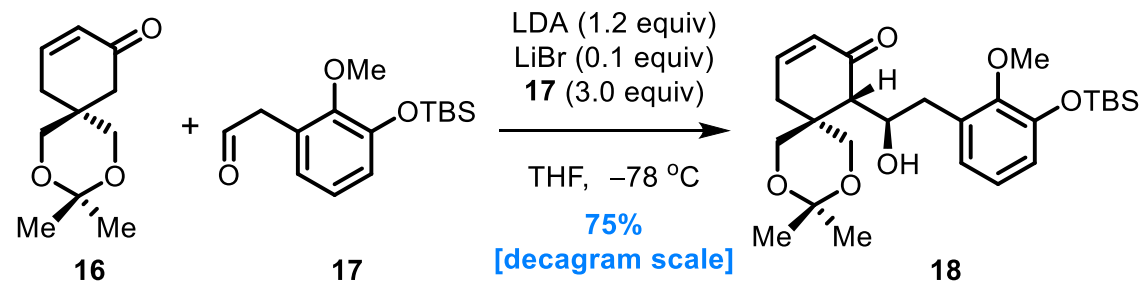

To a stirred solution of $i$ - $\operatorname{Pr}_{2} \mathrm{NH}\left(23.8 \mathrm{~mL}, 170 \mathrm{mmol}, 1.3\right.$ equiv) in THF $(90 \mathrm{~mL})$ at $-78{ }^{\circ} \mathrm{C}$ was added $n$-BuLi (62.8 mL, $2.5 \mathrm{M}$ in hexanes, $157 \mathrm{mmol}, 1.2$ equiv). The resulting mixture was 
stirred at $-78{ }^{\circ} \mathrm{C}$ for 20 min before it was warmed to $0{ }^{\circ} \mathrm{C}$ and stirred for an additional 15 min. The reaction was recooled to $-78{ }^{\circ} \mathrm{C}$ and sequentially added $\mathrm{LiBr}(1.11 \mathrm{~g}, 12.8 \mathrm{mmol}, 0.1$ equiv) and a solution of acetonide 16 (25.1 g, $128 \mathrm{mmol}, 1.0$ equiv) in THF (100 mL). The resulting mixture was stirred at $-78{ }^{\circ} \mathrm{C}$ for $1 \mathrm{~h}$ before it was added a solution of aldehyde $\mathbf{1 7}$ (109 g, 390 mmol, 3.0 equiv) in THF $(100 \mathrm{~mL})$. The reaction was stirred at $-78{ }^{\circ} \mathrm{C}$ for further $12 \mathrm{~h}$ before it was added $\mathrm{NH}_{4} \mathrm{Cl}(200 \mathrm{~mL}$, sat. aq.). The layers were separated, and the aqueous layer was extracted with EtOAc $(3 \times 100 \mathrm{~mL})$. The combined organic layers were washed with brine $(100$ $\mathrm{mL})$, dried $\left(\mathrm{Na}_{2} \mathrm{SO}_{4}\right)$ and concentrated in vacuo. Flash column chromatography (silica gel, hexanes:EtOAc 3:1) afforded homobenzylic alcohol 18 (45.7 g, 75\%) as a white amorphous solid. 18: $R_{\mathrm{f}}=0.45$ (silica gel, hexanes:EtOAc 3:1); ${ }^{1} \mathrm{H}$ NMR $\left(400 \mathrm{MHz}, \mathrm{CDCl}_{3}\right) \delta=7.00-6.83(\mathrm{~m}, 2$ H), $6.76(\mathrm{dd}, J=8.2,1.5 \mathrm{~Hz}, 1 \mathrm{H}), 6.70(\mathrm{dd}, J=7.6,1.6 \mathrm{~Hz}, 1 \mathrm{H}), 6.06(\mathrm{dd}, J=10.1,1.6 \mathrm{~Hz}, 1 \mathrm{H})$, 4.11-3.94 (m, $3 \mathrm{H}), 3.76$ (s, $3 \mathrm{H}), 3.65$ (d, $J=11.9 \mathrm{~Hz}, 1 \mathrm{H}), 3.61(\mathrm{~d}, J=10.7 \mathrm{~Hz}, 1 \mathrm{H}), 3.05$ (dd, $J=20.4,5.3 \mathrm{~Hz}, 1 \mathrm{H}), 2.90(\mathrm{~d}, J=4.5 \mathrm{~Hz}, 1 \mathrm{H}), 2.67(\mathrm{dd}, J=13.7,9.9 \mathrm{~Hz}, 1 \mathrm{H}), 2.58(\mathrm{dd}, J=$ 13.7, $2.4 \mathrm{~Hz}, 1 \mathrm{H}), 2.56-2.46(\mathrm{~m}, 1 \mathrm{H}), 2.35(\mathrm{~d}, J=7.9 \mathrm{~Hz}, 1 \mathrm{H}), 1.43(\mathrm{~s}, 3 \mathrm{H}), 1.39(\mathrm{~s}, 3 \mathrm{H}), 1.00$ (s, $9 \mathrm{H}), 0.18 \mathrm{ppm}(\mathrm{s}, 6 \mathrm{H}) ;{ }^{13} \mathrm{C} \mathrm{NMR}\left(100 \mathrm{MHz}, \mathrm{CDCl}_{3}\right) \delta=200.2,149.5,148.9,148.5,132.0$, $128.8,124.5,124.0,120.4,98.3,70.4,68.8,66.1,60.3,59.6,38.2,37.6,30.2,28.1,25.9(3 \mathrm{C})$, 19.5, 18.4, -4.4 ppm (2C); IR (film) $v_{\max }=2930,2858,1676,1473,1373,1288,1252,1203$, 1081, 1036, 835, $782 \mathrm{~cm}^{-1}$; HRMS (ESI): calcd for $\mathrm{C}_{26} \mathrm{H}_{40} \mathrm{NaO}_{6} \mathrm{Si}^{+}[\mathrm{M}+\mathrm{Na}]^{+}$499.2486, found 499.2498. 
Table S1. Optimization of the Acylative Kinetic Resolution of Homobenzylic Alcohol 18:

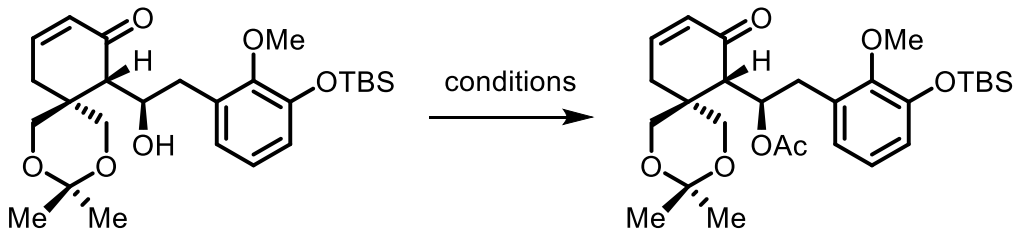

18

$(+)-20$

\begin{tabular}{|c|c|c|c|c|}
\hline \multirow[t]{2}{*}{ entry } & \multirow[t]{2}{*}{ conditions } & \multicolumn{2}{|c|}{$(+)-20$} & \multirow{2}{*}{$\begin{array}{c}\text { rec. } \\
18 \\
\text { yield } \\
(\%)^{c}\end{array}$} \\
\hline & & $\begin{array}{l}\text { yield } \\
(\%)^{c}\end{array}$ & $\mathbf{e r}^{d}$ & \\
\hline $1^{a}$ & $(R)-\mathrm{CF}_{3}-\mathrm{PIP}, \mathrm{Ac}_{2} \mathrm{O}, i-\mathrm{Pr}_{2} \mathrm{NEt}$, toluene, $40^{\circ} \mathrm{C}, 1 \mathrm{~d}$ & 26 & $73: 27$ & 52 \\
\hline $2^{a}$ & $(R)$-Cl-PIQ, $\mathrm{Ac}_{2} \mathrm{O}, i$ - $\operatorname{Pr}_{2} \mathrm{NEt}$, toluene, $40^{\circ} \mathrm{C}, 1 \mathrm{~d}$ & 31 & $61: 39$ & 51 \\
\hline $3^{a}$ & $(R)$-Tetramisole, $\mathrm{Ac}_{2} \mathrm{O}, i-\operatorname{Pr}_{2} \mathrm{NEt}$, toluene, $40^{\circ} \mathrm{C}, 1 \mathrm{~d}$ & 35 & $62: 38$ & 43 \\
\hline $4^{a}$ & $(R)-\mathrm{BTM}, \mathrm{Ac}_{2} \mathrm{O}, i-\mathrm{Pr}_{2} \mathrm{NEt}$, toluene, $40^{\circ} \mathrm{C}, 1 \mathrm{~d}$ & 37 & $70: 30$ & 50 \\
\hline $5^{b}$ & $(S)$-HBTM, $\mathrm{Ac}_{2} \mathrm{O}, i-\operatorname{Pr}_{2} \mathrm{NEt}$, toluene, $0{ }^{\circ} \mathrm{C}, 2 \mathrm{~d}$ & 44 & $77: 23$ & 47 \\
\hline $6^{b}$ & $(S)$-HBTM, $\mathrm{Ac}_{2} \mathrm{O}, i-\mathrm{Pr}_{2} \mathrm{NEt}, \mathrm{CH}_{2} \mathrm{Cl}_{2}, 0{ }^{\circ} \mathrm{C}, 5 \mathrm{~d}$ & $<5$ & - & 89 \\
\hline $7^{b}$ & $(S)$-HBTM, $\mathrm{Ac}_{2} \mathrm{O}, i-\mathrm{Pr}_{2} \mathrm{NEt}, \mathrm{Et}_{2} \mathrm{O}, 0{ }^{\circ} \mathrm{C}, 2 \mathrm{~d}$ & 42 & $71: 29$ & 45 \\
\hline $8^{b}$ & $(S)$-HBTM, $\mathrm{Ac}_{2} \mathrm{O}, i-\mathrm{Pr}_{2} \mathrm{NEt}, \mathrm{CHCl}_{3}, 0{ }^{\circ} \mathrm{C}, 5 \mathrm{~d}$ & $<5$ & - & 92 \\
\hline $9^{b}$ & $(S)$-HBTM, $\mathrm{Ac}_{2} \mathrm{O}, i-\mathrm{Pr}_{2} \mathrm{NEt}, \mathrm{MeCN}, 0{ }^{\circ} \mathrm{C}, 5 \mathrm{~d}$ & $<5$ & - & 90 \\
\hline $10^{b}$ & $\begin{array}{c}(S) \text {-HBTM, } \mathrm{Ac}_{2} \mathrm{O}, i-\mathrm{Pr}_{2} \mathrm{NEt} \\
t \text {-AmylOH/toluene }(1: 1), 0^{\circ} \mathrm{C}, 2 \mathrm{~d}\end{array}$ & 39 & $65: 35$ & 46 \\
\hline $11^{b}$ & $(S)-\mathrm{HBTM}, \mathrm{Ac}_{2} \mathrm{O}, i-\mathrm{Pr}_{2} \mathrm{NEt}$, toluene, $-20^{\circ} \mathrm{C}, 3 \mathrm{~d}$ & 42 & $82: 18$ & 44 \\
\hline $12^{b}$ & $(S)$-HBTM, $\mathrm{Ac}_{2} \mathrm{O}, i-\mathrm{Pr}_{2} \mathrm{NEt}$, toluene, $-40^{\circ} \mathrm{C}, 5 \mathrm{~d}$ & 41 & $85: 15$ & 49 \\
\hline $13^{b}$ & $(S)$-HBTM, $\mathrm{Ac}_{2} \mathrm{O}, i-\mathrm{Pr}_{2} \mathrm{NEt}$, toluene, $-60^{\circ} \mathrm{C}, 7 \mathrm{~d}$ & 38 & $89: 11$ & 50 \\
\hline $14^{b}$ & $(S)-\mathrm{Me}-\mathrm{HBTM}, \mathrm{Ac}_{2} \mathrm{O}, i-\mathrm{Pr}_{2} \mathrm{NEt}$, toluene, $-60^{\circ} \mathrm{C}, 7 \mathrm{~d}$ & 40 & $79: 21$ & 47 \\
\hline $15^{b}$ & $(S)-\mathrm{MeO}-\mathrm{HBTM}, \mathrm{Ac}_{2} \mathrm{O}, i-\mathrm{Pr}_{2} \mathrm{NEt}$, toluene, $-60{ }^{\circ} \mathrm{C}, 7 \mathrm{~d}$ & 46 & $76: 24$ & 45 \\
\hline $16^{b}$ & $(S)$-Br-HBTM, $\mathrm{Ac}_{2} \mathrm{O}, i$ - $\operatorname{Pr}_{2} \mathrm{NEt}$, toluene, $-60^{\circ} \mathrm{C}, 7 \mathrm{~d}$ & 31 & $81: 19$ & 55 \\
\hline $17^{b, e}$ & $(S)-\mathrm{CF}_{3}-\mathrm{HBTM}(\mathbf{1 9}), \mathrm{Ac}_{2} \mathrm{O}, i-\mathrm{Pr}_{2} \mathrm{NEt}$, toluene, $-60{ }^{\circ} \mathrm{C}, 7 \mathrm{~d}$ & 43 & $91: 9$ & 48 \\
\hline $18^{b}$ & $(S)-\mathrm{NO}_{2}-\mathrm{HBTM}, \mathrm{Ac}_{2} \mathrm{O}, i-\mathrm{Pr}_{2} \mathrm{NEt}$, toluene, $-60^{\circ} \mathrm{C}, 7 \mathrm{~d}$ & $<5$ & - & 93 \\
\hline $19^{b}$ & $(S)-\mathrm{CO}_{2} \mathrm{Me}-\mathrm{HBTM}, \mathrm{Ac}_{2} \mathrm{O}, i-\mathrm{Pr}_{2} \mathrm{NEt}$, toluene, $-60^{\circ} \mathrm{C}, 7 \mathrm{~d}$ & 31 & $78: 22$ & 58 \\
\hline $20^{b}$ & $(2 S, 3 R)$-HyperBTM, $\mathrm{Ac}_{2} \mathrm{O}, i$ - $\operatorname{Pr}_{2} \mathrm{NEt}$, toluene, $-60^{\circ} \mathrm{C}, 7 \mathrm{~d}$ & 42 & $69: 31$ & 50 \\
\hline $21^{b}$ & (4R)-Mes-DHPB, $\mathrm{Ac}_{2} \mathrm{O}, i$ - $\mathrm{Pr}_{2} \mathrm{NEt}$, toluene, $-60^{\circ} \mathrm{C}, 7 \mathrm{~d}$ & 35 & $74: 26$ & 52 \\
\hline
\end{tabular}

${ }^{a}$ Condition A: $18(0.10 \mathrm{mmol})$, chiral base (1.0 equiv), $\mathrm{Ac}_{2} \mathrm{O}$ (1.0 equiv), $i$ - $\operatorname{Pr}_{2} \mathrm{NEt}(1.0$ equiv), solvent $(2 \mathrm{~mL}) .{ }^{b}$ Condition $\mathrm{B}: 18(0.10 \mathrm{mmol})$, chiral base $\left(0.10\right.$ equiv), $\mathrm{Ac}_{2} \mathrm{O}$ (0.60 equiv), $i$ - $\operatorname{Pr}_{2}$ NEt $\left(0.60\right.$ equiv), solvent $(2 \mathrm{~mL}) .{ }^{c}$ Isolated yields. ${ }^{d}$ Determined by HPLC analysis. 
${ }^{e}$ Decagram scale.

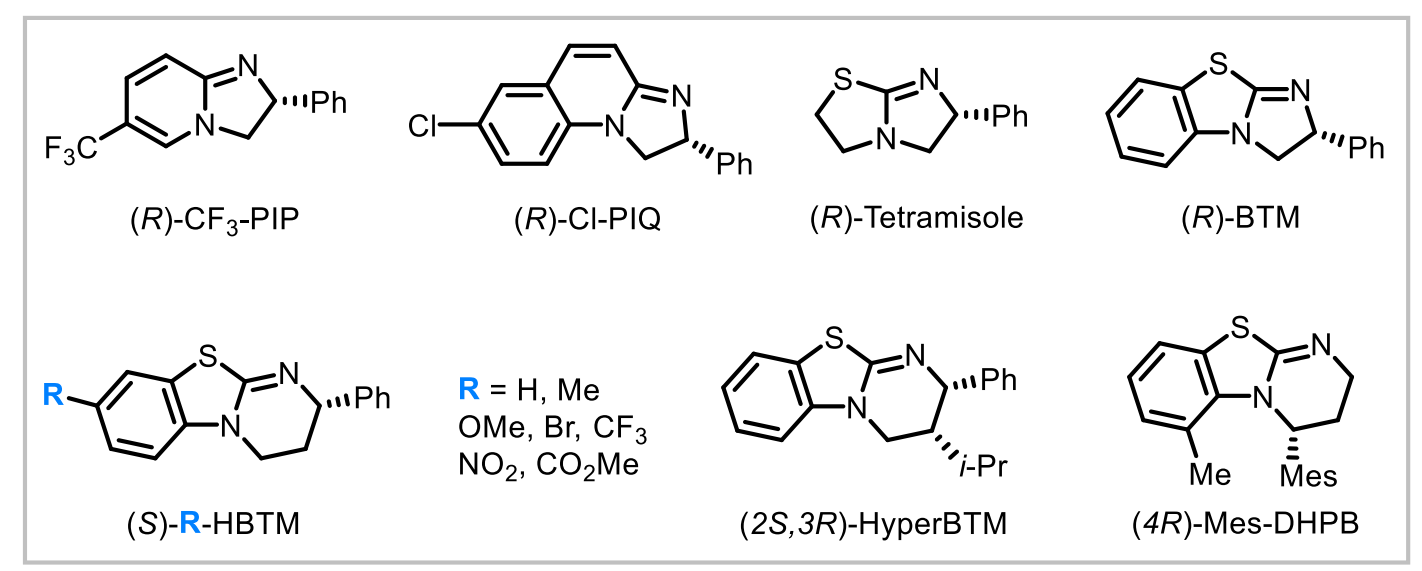

\section{Preparation of acetate (+)-20 (entry 17, Table S1):}

To a stirred solution of homobenzylic alcohol 18 (42.8 g, 90.0 mmol, 1.0 equiv) in toluene $(1.8 \mathrm{~L})$ at $-60{ }^{\circ} \mathrm{C}$ were sequentially added $i$ - $\operatorname{Pr}_{2} \mathrm{NEt}(9.41 \mathrm{~mL}, 54.0 \mathrm{mmol}, 0.6$ equiv), (S)- $\mathrm{CF}_{3}-\mathrm{HBTM} 19$ (3.01 g, $9.00 \mathrm{mmol}, 0.1$ equiv) and $\mathrm{Ac}_{2} \mathrm{O}$ (5.10 mL, $54.0 \mathrm{mmol}, 0.6$ equiv). The resulting mixture was stirred at $-60{ }^{\circ} \mathrm{C}$ for $7 \mathrm{~d}$ before it was added $\mathrm{NaHCO}_{3}(500 \mathrm{~mL}$, sat. aq.). The layers were separated, and the aqueous layer was extracted with EtOAc $(3 \times 200 \mathrm{~mL})$. The combined organic layers were dried $\left(\mathrm{Na}_{2} \mathrm{SO}_{4}\right)$ and concentrated in vacuo. Flash column chromatography (silica gel, hexanes:EtOAc 3:1) afforded acetate (+)-20 (20.1 g, 43\%) as a colorless oil, along with the recovered alcohol $18(20.6 \mathrm{~g}, 48 \%) .(+)-20:[\alpha]_{\mathrm{D}}^{25}=+71.1(\mathrm{c}=1.0$, $\left.\mathrm{CHCl}_{3}\right) ; 91: 9$ er [Daicel Chiralpak IB $(0.46 \mathrm{~cm} \times 25 \mathrm{~cm}), n$-hexane/2-propanol $=99 / 1, \mathrm{v}=0.5$ $\mathrm{mL} \cdot \min ^{-1}, \lambda=210 \mathrm{~nm}, \mathrm{t}($ major $)=23.23 \mathrm{~min}, \mathrm{t}($ minor $\left.)=22.06 \mathrm{~min}\right]$.

\section{Recycle of the recovered homobenzylic alcohol 18:}

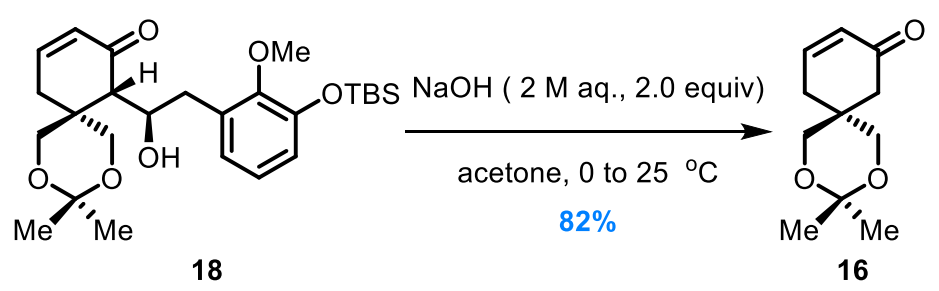


To a stirred solution of the recovered homobenzylic alcohol 18 (15.0 g, 31.5 mmol, 1.0 equiv) in acetone $(150 \mathrm{~mL})$ at $0{ }^{\circ} \mathrm{C}$ was added $\mathrm{NaOH}(31.5 \mathrm{~mL}, 2.0 \mathrm{M}$ aq., $63.0 \mathrm{mmol}, 2.0$ equiv). The resulting mixture was warmed to $25{ }^{\circ} \mathrm{C}$ and stirred for $4 \mathrm{~h}$ before it was added $\mathrm{NH}_{4} \mathrm{Cl}(50 \mathrm{~mL}$, sat. aq.). The layers were separated, and the aqueous layer was extracted with $\mathrm{Et}_{2} \mathrm{O}(3 \times 20 \mathrm{~mL})$. The combined organic layers were dried $\left(\mathrm{Na}_{2} \mathrm{SO}_{4}\right)$ and concentrated in vacuo. Flash column chromatography (silica gel, hexanes:EtOAc 2:1) afforded acetonide 16 (5.06 g, 82\%).

\section{Preparation of acetate $( \pm)-20:$}
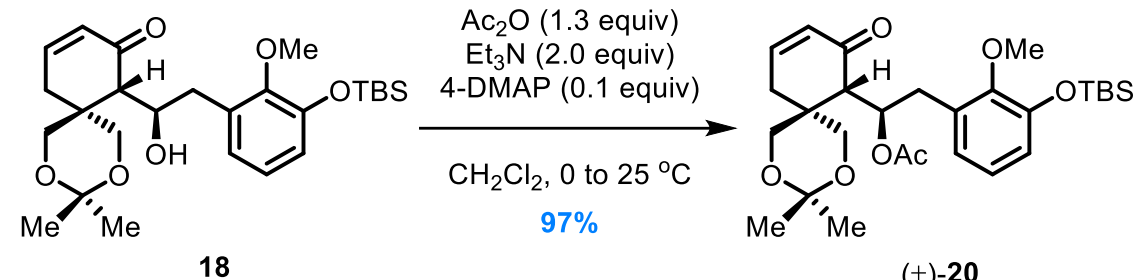

$( \pm)-20$

To a stirred solution of homobenzylic alcohol 18 (2.0 g, $4.2 \mathrm{mmol}, 1.0$ equiv) in $\mathrm{CH}_{2} \mathrm{Cl}_{2}$ (40 $\mathrm{mL})$ at $0{ }^{\circ} \mathrm{C}$ were sequentially added $\mathrm{Et}_{3} \mathrm{~N}\left(1.2 \mathrm{~mL}, 8.4 \mathrm{mmol}, 2.0\right.$ equiv), $\mathrm{Ac}_{2} \mathrm{O}(0.52 \mathrm{~mL}, 5.5$ mmol, 1.3 equiv) and 4-DMAP (51 $\mathrm{mg}, 0.42 \mathrm{mmol}, 0.1$ equiv). The resulting mixture was warmed to $25{ }^{\circ} \mathrm{C}$ and stirred for $2 \mathrm{~h}$ before it was added $\mathrm{NaHCO}_{3}(20 \mathrm{~mL}$, sat. aq. $)$. The layers were separated, and the aqueous layer was extracted with $\mathrm{CH}_{2} \mathrm{Cl}_{2}(3 \times 10 \mathrm{~mL})$. The combined organic layers were dried $\left(\mathrm{Na}_{2} \mathrm{SO}_{4}\right)$ and concentrated in vacuo. Flash column chromatography (silica gel, hexanes:EtOAc 3:1) afforded acetate $( \pm)-\mathbf{2 0}(2.1 \mathrm{~g}, 97 \%)$ as a colorless oil. $( \pm)-\mathbf{2 0}: R_{\mathrm{f}}=$ 0.50 (silica gel, hexanes:EtOAc 3:1); ${ }^{1} \mathrm{H}$ NMR $\left(400 \mathrm{MHz}, \mathrm{CDCl}_{3}\right) \delta=6.98-6.89(\mathrm{~m}, 1 \mathrm{H}), 6.80(\mathrm{t}$, $J=7.8 \mathrm{~Hz}, 1 \mathrm{H}), 6.70(\mathrm{~d}, J=7.9 \mathrm{~Hz}, 1 \mathrm{H}), 6.67(\mathrm{~d}, J=7.5 \mathrm{~Hz}, 1 \mathrm{H}), 6.08(\mathrm{~d}, J=10.2 \mathrm{~Hz}, 1 \mathrm{H})$, 5.50-5.32 (m, $1 \mathrm{H}), 3.98(\mathrm{~d}, J=11.7 \mathrm{~Hz}, 1 \mathrm{H}), 3.76(\mathrm{~s}, 3 \mathrm{H}), 3.73(\mathrm{~d}, J=11.7 \mathrm{~Hz}, 1 \mathrm{H}), 3.66-3.56$ (m, $2 \mathrm{H}), 3.03(\mathrm{dd}, J=20.4,5.3 \mathrm{~Hz}, 1 \mathrm{H}), 2.85(\mathrm{dd}, J=13.6,2.9 \mathrm{~Hz}, 1 \mathrm{H}), 2.66-2.48(\mathrm{~m}, 3 \mathrm{H})$, 1.83 (s, $3 \mathrm{H}), 1.43$ (s, $3 \mathrm{H}), 1.39$ (s, $3 \mathrm{H}), 0.99$ (s, $9 \mathrm{H}), 0.17$ (s, $3 \mathrm{H}), 0.14$ ppm (s, $3 \mathrm{H}) ;{ }^{13} \mathrm{C} \mathrm{NMR}$ $\left(100 \mathrm{MHz}, \mathrm{CDCl}_{3}\right) \delta=198.2,169.9,150.0,148.6,148.0,130.8,129.0,124.2,123.4,120.5,98.4$, 
$70.4,67.7,66.0,60.1,56.1,37.7,34.4,30.2,28.0,25.9$ (3C), 21.3, 19.4, 18.4, -4.4, -4.5 ppm; IR (film) $v_{\max }=2931,2859,1742,1682,1474,1373,1288,1252,1220,1084,1024,1036,840,750$ $\mathrm{cm}^{-1}$; HRMS (ESI): calcd for $\mathrm{C}_{28} \mathrm{H}_{42} \mathrm{NaO}_{7} \mathrm{Si}^{+}[\mathrm{M}+\mathrm{Na}]^{+}$541.2592, found 541.2599.

\section{Preparation of $(S)-C_{3}-{ }_{3 B T M} 19:^{3}$}

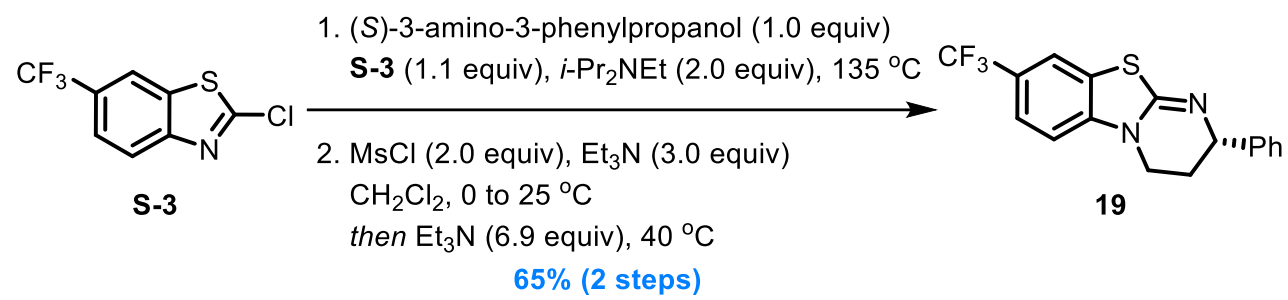

To a stirred solution of 2-chloro-6-trifluoromethylbenzothiazole (S-3, 5.9 g, 25 mmol, 1.1 equiv) in $i$ - $\operatorname{Pr}_{2} \mathrm{NEt}\left(8.0 \mathrm{~mL}, 46 \mathrm{mmol}, 2.0\right.$ equiv) at $25{ }^{\circ} \mathrm{C}$ was added $(S)$-3-amino-3-phenylpropanol (3.5 g, $23 \mathrm{mmol}, 1.0$ equiv). The resulting mixture was warmed to $135{ }^{\circ} \mathrm{C}$ and stirred for $12 \mathrm{~h}$ before it was cooled to $25^{\circ} \mathrm{C}$ and concentrated in vacuo to afforded the crude alcohol as a white amorphous solid, which was used without further purification.

To a stirred solution of the alcohol (crude, obtained above) in $\mathrm{CH}_{2} \mathrm{Cl}_{2}(60 \mathrm{~mL})$ at $0{ }^{\circ} \mathrm{C}$ were sequentially added $\mathrm{Et}_{3} \mathrm{~N}$ (9.6 mL, 69 mmol, 3.0 equiv) and $\mathrm{MsCl}$ (3.6 mL, 46 mmol, 2.0 equiv). The resulting mixture was warmed to $25^{\circ} \mathrm{C}$ and stirred for $1 \mathrm{~h}$ before it was added $\mathrm{MeOH}(2 \mathrm{~mL})$. The reaction was stirred for an additional $1 \mathrm{~h}$ before it was added $\mathrm{Et}_{3} \mathrm{~N}(22 \mathrm{~mL}, 159 \mathrm{mmol}, 6.9$ equiv). The resulting mixture was warmed to $40{ }^{\circ} \mathrm{C}$ and stirred for $12 \mathrm{~h}$ before it was cooled to 25 ${ }^{\circ} \mathrm{C}$ and added $\mathrm{NaHCO}_{3}(30 \mathrm{~mL}$, sat. aq.). The layers were separated, and the aqueous layer was extracted with $\mathrm{CH}_{2} \mathrm{Cl}_{2}(3 \times 20 \mathrm{~mL})$. The combined organic layers were dried $\left(\mathrm{Na}_{2} \mathrm{SO}_{4}\right)$ and concentrated in vacuo. Flash column chromatography (silica gel, hexanes:EtOAc 4:1) afforded $(S)-\mathrm{CF}_{3}-\mathrm{HBTM} 19\left(5.0 \mathrm{~g}, 65 \%\right.$ over two steps) as a yellow amorphous solid. 19: $[\alpha]_{\mathrm{D}}{ }^{25}=+29.8(\mathrm{c}$ $\left.=1.0, \mathrm{CHCl}_{3}\right) ;{ }^{1} \mathrm{H} \mathrm{NMR}\left(400 \mathrm{MHz}, \mathrm{CDCl}_{3}\right) \delta=7.56(\mathrm{~d}, J=1.7 \mathrm{~Hz}, 1 \mathrm{H}), 7.46(\mathrm{ddd}, J=8.4,1.8$, $0.9 \mathrm{~Hz}, 1 \mathrm{H}), 7.40-7.31(\mathrm{~m}, 4 \mathrm{H}), 7.30-7.25(\mathrm{~m}, 1 \mathrm{H}), 6.79$ (d, $J=8.3 \mathrm{~Hz}, 1 \mathrm{H}), 4.74(\mathrm{dd}, J=8.1$, 
$4.0 \mathrm{~Hz}, 1 \mathrm{H}), 3.86(\mathrm{ddd}, J=11.6,8.8,4.8 \mathrm{~Hz}, 1 \mathrm{H}), 3.74(\mathrm{dt}, J=11.3,5.3 \mathrm{~Hz}, 1 \mathrm{H}), 2.39-2.28(\mathrm{~m}$, $1 \mathrm{H}), 2.09-1.94 \mathrm{ppm}(\mathrm{m}, 1 \mathrm{H}) ;{ }^{13} \mathrm{C} \mathrm{NMR}\left(100 \mathrm{MHz}, \mathrm{CDCl}_{3}\right) \delta=157.4,143.7,143.2,128.6(2 \mathrm{C})$, 127.2, $126.6(2 \mathrm{C}), 124.3\left(\mathrm{q}, J_{\mathrm{C}-\mathrm{F}}=270 \mathrm{~Hz}\right), 124.2\left(\mathrm{q}, J_{\mathrm{C}-\mathrm{F}}=33 \mathrm{~Hz}\right), 123.6\left(\mathrm{q}, J_{\mathrm{C}-\mathrm{F}}=4.0 \mathrm{~Hz}\right)$, 123.5, 119.1 (q, $\left.J_{\mathrm{C}-\mathrm{F}}=3.9 \mathrm{~Hz}\right), 107.0,58.6,40.9,27.8$ ppm; IR (film) $v_{\max }=1626,1602,1586$, 1361, 1285, 1159, 814, $697 \mathrm{~cm}^{-1}$; HRMS (ESI): calcd for $\mathrm{C}_{17} \mathrm{H}_{13} \mathrm{~F}_{3} \mathrm{~N}_{2} \mathrm{NaS}^{+}[\mathrm{M}+\mathrm{Na}]^{+} 357.0644$, found 357.0647 .

\section{Preparation of vinyl triflate 21:}

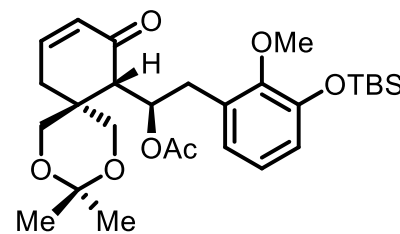

$(+)-20$

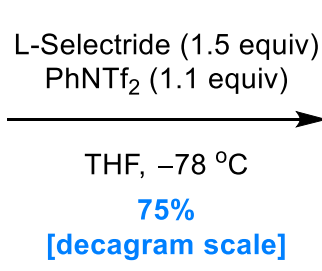

[decagram scale]

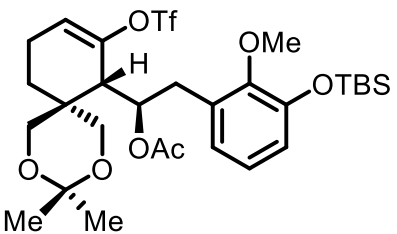

21

To a stirred solution of acetate (+)-20 (18.5 g, $35.7 \mathrm{mmol}, 1.0$ equiv) in THF (150 mL) at -78 ${ }^{\circ} \mathrm{C}$ was added L-Selectride (53.6 mL, 1.0 M in THF, $53.6 \mathrm{mmol}, 1.5$ equiv). The resulting mixture was stirred at $-78{ }^{\circ} \mathrm{C}$ for $0.5 \mathrm{~h}$ before it was added a solution of $\mathrm{PhNTf}_{2}(14.0 \mathrm{~g}, 39.3 \mathrm{mmol}, 1.1$ equiv) in THF $(50 \mathrm{~mL})$. The reaction was stirred at $-78{ }^{\circ} \mathrm{C}$ for an additional $2 \mathrm{~h}$ before it was added $\mathrm{NH}_{4} \mathrm{Cl}$ (100 mL, sat. aq.). The layers were separated, and the aqueous layer was extracted with EtOAc $(3 \times 50 \mathrm{~mL})$. The combined organic layers were washed with brine $(50 \mathrm{~mL})$, dried $\left(\mathrm{Na}_{2} \mathrm{SO}_{4}\right)$ and concentrated in vacuo. Flash column chromatography (silica gel, hexanes:EtOAc 10:1) afforded vinyl triflate $21(17.5 \mathrm{~g}, 75 \%)$ as a colorless oil. 21: $R_{\mathrm{f}}=0.50$ (silica gel, hexanes:EtOAc 10:1); $[\alpha]_{\mathrm{D}}{ }^{25}=+45.0\left(\mathrm{c}=0.50, \mathrm{CHCl}_{3}\right) ;{ }^{1} \mathrm{H} \mathrm{NMR}\left(400 \mathrm{MHz}, \mathrm{CDCl}_{3}\right) \delta=6.83(\mathrm{t}$, $J=7.8 \mathrm{~Hz}, 1 \mathrm{H}), 6.79-6.65(\mathrm{dd}, J=7.5,2.0 \mathrm{~Hz}, 2 \mathrm{H}), 5.96(\mathrm{t}, J=3.9 \mathrm{~Hz}, 1 \mathrm{H}), 5.28(\mathrm{dt}, J=11.3$, $2.6 \mathrm{~Hz}, 1 \mathrm{H}), 4.21(\mathrm{~d}, J=11.7 \mathrm{~Hz}, 1 \mathrm{H}), 3.83(\mathrm{~s}, 3 \mathrm{H}), 3.77(\mathrm{~d}, J=11.8 \mathrm{~Hz}, 1 \mathrm{H}), 3.69(\mathrm{~d}, J=11.9$ Hz, $1 \mathrm{H}), 3.61(\mathrm{~d}, J=11.9 \mathrm{~Hz}, 1 \mathrm{H}), 2.92(\mathrm{dd}, J=13.7,2.9 \mathrm{~Hz}, 1 \mathrm{H}), 2.79-2.62(\mathrm{~m}, 2 \mathrm{H}), 2.40-$ $2.22(\mathrm{~m}, 2 \mathrm{H}), 2.19-2.06(\mathrm{~m}, 1 \mathrm{H}), 1.88$ (s, $3 \mathrm{H}), 1.68-1.58(\mathrm{~m}, 1 \mathrm{H}), 1.46(\mathrm{~s}, 3 \mathrm{H}), 1.42(\mathrm{~s}, 3 \mathrm{H})$, 
$1.00(\mathrm{~s}, 9 \mathrm{H}), 0.18(\mathrm{~s}, 3 \mathrm{H}), 0.15 \mathrm{ppm}(\mathrm{s}, 3 \mathrm{H}) ;{ }^{13} \mathrm{C} \mathrm{NMR}\left(100 \mathrm{MHz}, \mathrm{CDCl}_{3}\right) \delta=170.2,149.7$, 148.6, 147.4, 131.2, 124.0, 123.5, 121.0, 120.4, $118.6\left(\mathrm{q}, J_{\mathrm{C}-\mathrm{F}}=320.5 \mathrm{~Hz}\right), 98.7,71.5,66.6(2 \mathrm{C})$, $60.2,46.1,36.8,33.8,26.1,25.9$ (3C), 22.5, 21.6, 20.9, 20.5, 18.4, -4.4, -4.5 ppm; IR (film) $v_{\max }$ $=2932,2872,1743,1474,1418,1288,1208,1143,839,772 \mathrm{~cm}^{-1}$; HRMS (ESI): calcd for $\mathrm{C}_{29} \mathrm{H}_{43} \mathrm{~F}_{3} \mathrm{NaO}_{9} \mathrm{SSi}^{+}[\mathrm{M}+\mathrm{Na}]^{+}$675.2241, found 675.2249.

\section{Preparation of phenol 14:}
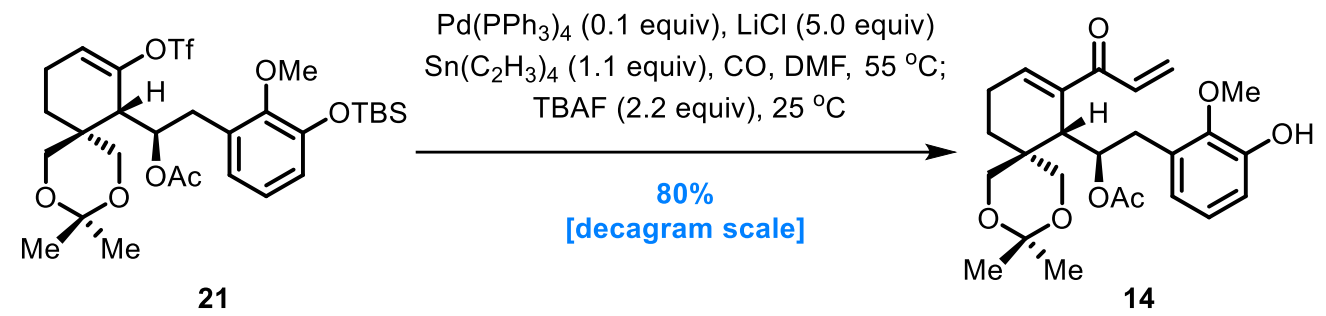

To a stirred solution of vinyl triflate 21 (12.0 g, 18.4 mmol, 1.0 equiv) in DMF (500 mL) at $25{ }^{\circ} \mathrm{C}$ were sequentially added $\mathrm{LiCl}\left(3.90 \mathrm{~g}, 92.0 \mathrm{mmol}, 5.0\right.$ equiv) and $\mathrm{Pd}\left(\mathrm{PPh}_{3}\right)_{4}(2.13 \mathrm{~g}, 1.84$ mmol, 0.1 equiv). The resulting mixture was stirred under $\mathrm{CO}$ atmosphere ( $1 \mathrm{~atm})$ for $0.5 \mathrm{~h}$ before it was added $\mathrm{Sn}\left(\mathrm{C}_{2} \mathrm{H}_{3}\right)_{4}\left(3.51 \mathrm{~mL}, 19.3 \mathrm{mmol}, 1.1\right.$ equiv). The reaction was warmed to $55^{\circ} \mathrm{C}$ and stirred for an additional $12 \mathrm{~h}$ before it was cooled to $25{ }^{\circ} \mathrm{C}$, exposed to argon atmosphere, and added THF (100 mL) and TBAF (39.5 mL, 1.0 M in THF, $39.5 \mathrm{mmol}, 2.2$ equiv). The resulting mixture was stirred for further $0.5 \mathrm{~h}$ before it was added $\mathrm{NH}_{4} \mathrm{Cl}$ (50 mL, sat. aq.). The layers were separated, and the aqueous layer was extracted with EtOAc $(3 \times 30 \mathrm{~mL})$. The combined organic layers were washed with brine $(30 \mathrm{~mL})$, dried $\left(\mathrm{Na}_{2} \mathrm{SO}_{4}\right)$ and concentrated in vacuo. Flash column chromatography (silica gel, hexanes:EtOAc 2:1) afforded phenol $14(6.54 \mathrm{~g}, 80 \%)$ as a colorless oil. 14: $R_{\mathrm{f}}=0.25$ (silica gel, hexanes:EtOAc $\left.2: 1\right) ;[\alpha]_{\mathrm{D}}{ }^{25}=+80.1\left(\mathrm{c}=0.50, \mathrm{CHCl}_{3}\right) ;{ }^{1} \mathrm{H} \mathrm{NMR}(400$ $\left.\mathrm{MHz}, \mathrm{CDCl}_{3}\right) \delta=6.88-6.74(\mathrm{~m}, 4 \mathrm{H}), 6.56(\mathrm{~d}, J=7.6 \mathrm{~Hz}, 1 \mathrm{H}), 6.30(\mathrm{~d}, J=17.1 \mathrm{~Hz}, 1 \mathrm{H}), 5.82$ $(\mathrm{d}, J=10.6 \mathrm{~Hz}, 1 \mathrm{H}), 5.72(\mathrm{~s}, 1 \mathrm{H}), 5.22(\mathrm{dt}, J=11.4,3.6 \mathrm{~Hz}, 1 \mathrm{H}), 4.17(\mathrm{~d}, J=11.6 \mathrm{~Hz}, 1 \mathrm{H})$, 
$3.76(\mathrm{~s}, 3 \mathrm{H}), 3.71(\mathrm{~d}, J=11.8 \mathrm{~Hz}, 1 \mathrm{H}), 3.52(\mathrm{~d}, J=11.9 \mathrm{~Hz}, 1 \mathrm{H}), 3.39(\mathrm{~d}, J=11.8 \mathrm{~Hz}, 1 \mathrm{H})$, $3.26(\mathrm{~d}, J=4.2 \mathrm{~Hz}, 1 \mathrm{H}), 2.82(\mathrm{dd}, J=13.9,1.9 \mathrm{~Hz}, 1 \mathrm{H}), 2.63(\mathrm{dd}, J=13.6,11.8 \mathrm{~Hz}, 1 \mathrm{H}), 2.52-$ $2.39(\mathrm{~m}, 1 \mathrm{H}), 2.38-2.21(\mathrm{~m}, 2 \mathrm{H}), 1.76(\mathrm{~s}, 3 \mathrm{H}), 1.73-1.64$ (m, $1 \mathrm{H}), 1.45$ (s, $3 \mathrm{H}), 1.41 \mathrm{ppm}(\mathrm{s}, 3$ $\mathrm{H}) ;{ }^{13} \mathrm{C} \mathrm{NMR}\left(100 \mathrm{MHz}, \mathrm{CDCl}_{3}\right) \delta=192.6,170.1,149.0,145.8,140.5,138.3,132.9,131.0$, $129.2,124.5,122.6,114.8,98.3,73.3,68.0,66.4,61.1,39.8,34.4,33.5,26.9,22.8,22.1,21.0$, 20.9 ppm; IR (film) $v_{\max }=3392,2962,2867,1738,1654,1606,1473,1372,1234,1197,1024$, 833, $753 \mathrm{~cm}^{-1}$; HRMS (ESI): calcd for $\mathrm{C}_{25} \mathrm{H}_{32} \mathrm{NaO}_{7}^{+}[\mathrm{M}+\mathrm{Na}]^{+}$467.2040, found 467.2046.

\section{Preparation of tetracyclic diene 22:}

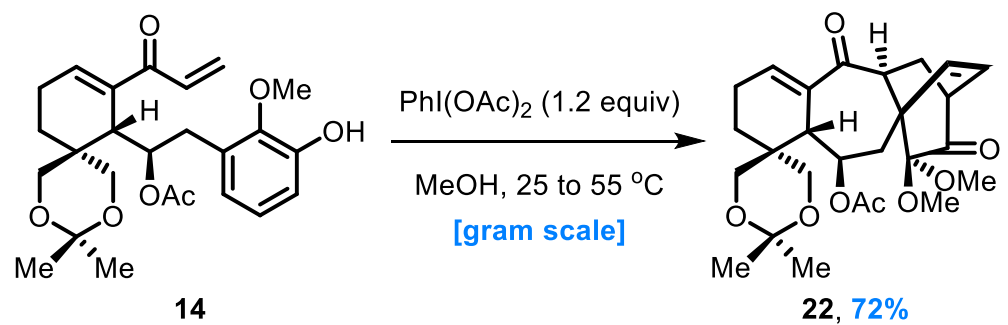

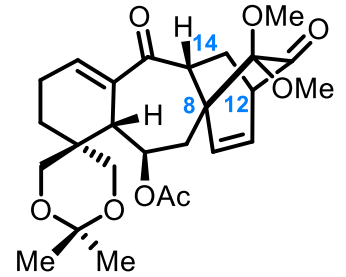

C8,C12,C14-triepi-22, 2.2\%
II

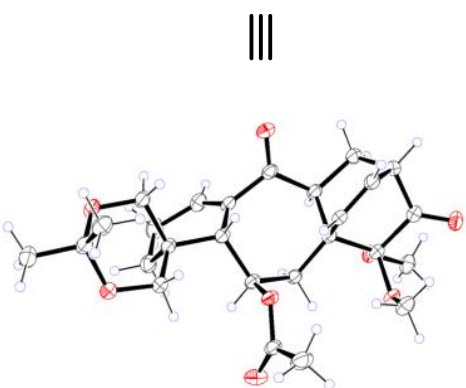

X-ray of 22 [CCDC 2086698]

To a stirred solution of phenol $14\left(2.7 \mathrm{~g}, 6.0 \mathrm{mmol}, 1.0\right.$ equiv) in $\mathrm{MeOH}(50 \mathrm{~mL})$ at $25{ }^{\circ} \mathrm{C}$ was added $\mathrm{PhI}(\mathrm{OAc})_{2}(2.3 \mathrm{~g}, 7.2 \mathrm{mmol}, 1.2$ equiv). The resulting mixture was stirred for $0.5 \mathrm{~h}$ before it was added $\mathrm{MeOH}(1.0 \mathrm{~L})$. The reaction was warmed to $55^{\circ} \mathrm{C}$ and stirred for $12 \mathrm{~h}$ before it was cooled to $25{ }^{\circ} \mathrm{C}$ and concentrated in vacuo. Flash column chromatography (silica gel, hexanes:EtOAc 2:1) afforded tetracyclic diene $22(2.1 \mathrm{~g}, 72 \%)$ as a white solid, along with its C8,C12,C14-triepimer (63 mg, 2.2\%) as a white amorphous solid. 22: CCDC 2086698; m.p. 165$166{ }^{\circ} \mathrm{C}\left(\mathrm{CH}_{2} \mathrm{Cl}_{2} /\right.$ hexanes); $R_{\mathrm{f}}=0.45$ (silica gel, hexanes:EtOAc $\left.2: 1\right) ;[\alpha]_{\mathrm{D}}{ }^{25}=-106.9(\mathrm{c}=0.40$, 
$\left.\mathrm{CHCl}_{3}\right) ;{ }^{1} \mathrm{H} \mathrm{NMR}\left(400 \mathrm{MHz}, \mathrm{CDCl}_{3}\right) \delta=6.67(\mathrm{~d}, J=8.4 \mathrm{~Hz}, 1 \mathrm{H}), 6.17(\mathrm{t}, J=7.6 \mathrm{~Hz}, 1 \mathrm{H}), 5.81$ $(\mathrm{t}, J=3.4 \mathrm{~Hz}, 1 \mathrm{H}), 5.35(\mathrm{dt}, J=7.7,3.5 \mathrm{~Hz}, 1 \mathrm{H}), 3.75(\mathrm{~d}, J=11.7 \mathrm{~Hz}, 1 \mathrm{H}), 3.60(\mathrm{~d}, J=11.9 \mathrm{~Hz}$, $1 \mathrm{H}), 3.51(\mathrm{~d}, J=11.9 \mathrm{~Hz}, 1 \mathrm{H}), 3.36(\mathrm{~s}, 3 \mathrm{H}), 3.32(\mathrm{~d}, J=11.8 \mathrm{~Hz}, 1 \mathrm{H}), 3.25-3.20(\mathrm{~m}, 1 \mathrm{H}), 3.18$ (s, $3 \mathrm{H}), 3.06(\mathrm{dd}, J=9.2,3.8 \mathrm{~Hz}, 1 \mathrm{H}), 2.77(\mathrm{dd}, J=16.7,2.8 \mathrm{~Hz}, 1 \mathrm{H}), 2.46(\mathrm{dt}, J=13.6,3.9 \mathrm{~Hz}$, $1 \mathrm{H}), 2.34(\mathrm{~d}, J=8.2 \mathrm{~Hz}, 1 \mathrm{H}), 2.25-2.02(\mathrm{~m}, 4 \mathrm{H}), 2.06(\mathrm{~s}, 3 \mathrm{H}), 1.89(\mathrm{dd}, J=13.1,9.5 \mathrm{~Hz}, 1 \mathrm{H})$, 1.54-1.43 (m, $1 \mathrm{H}), 1.38(\mathrm{~s}, 3 \mathrm{H}), 1.37 \mathrm{ppm}(\mathrm{s}, 3 \mathrm{H}) ;{ }^{13} \mathrm{C} \mathrm{NMR}\left(100 \mathrm{MHz}, \mathrm{CDCl}_{3}\right) \delta=206.9$, $201.4,169.8,139.2,134.9,129.1,127.8,98.2,95.8,72.5,67.3,65.8,55.1,53.3,52.2,49.4,47.5$, 40.9, 34.9, 32.5, 26.0, 22.9, 21.7, 21.6 (2C), 21.4 ppm; IR (film) $v_{\max }=2941,2874,1736,1690$, 1455, 1373, 1237, 1201, 1101, 1024, 833, $733 \mathrm{~cm}^{-1}$; HRMS (ESI): calcd for $\mathrm{C}_{26} \mathrm{H}_{34} \mathrm{NaO}_{8}{ }^{+}[\mathrm{M}+$ $\mathrm{Na}]^{+}$497.2146, found 497.2151.

C8,C12,C14-triepi-22: $R_{\mathrm{f}}=0.35$ (silica gel, hexanes:EtOAc 2:1); $[\alpha]_{\mathrm{D}}^{25}=+189.1(\mathrm{c}=0.50$, $\left.\mathrm{CHCl}_{3}\right) ;{ }^{1} \mathrm{H} \mathrm{NMR}\left(400 \mathrm{MHz}, \mathrm{CDCl}_{3}\right) \delta=7.04(\mathrm{t}, J=3.6 \mathrm{~Hz}, 1 \mathrm{H}), 6.18(\mathrm{t}, J=7.5 \mathrm{~Hz}, 1 \mathrm{H}), 5.56$ $(\mathrm{d}, J=8.0 \mathrm{~Hz}, 1 \mathrm{H}), 4.98(\mathrm{dt}, J=7.2,3.6 \mathrm{~Hz}, 1 \mathrm{H}), 4.21(\mathrm{dd}, J=8.8,3.7 \mathrm{~Hz}, 1 \mathrm{H}), 3.83(\mathrm{~d}, J=$ $11.9 \mathrm{~Hz}, 1 \mathrm{H}), 3.55(\mathrm{~d}, J=11.9 \mathrm{~Hz}, 1 \mathrm{H}), 3.49(\mathrm{~d}, J=11.9 \mathrm{~Hz}, 1 \mathrm{H}), 3.40(\mathrm{~s}, 3 \mathrm{H}), 3.39(\mathrm{~d}, J=$ $11.7 \mathrm{~Hz}, 1 \mathrm{H}), 3.25(\mathrm{ddd}, J=7.2,3.6,1.8 \mathrm{~Hz}, 1 \mathrm{H}), 3.15(\mathrm{~s}, 3 \mathrm{H}), 3.02-2.91(\mathrm{~m}, 2 \mathrm{H}), 2.44(\mathrm{dt}, J=$ 13.1, 3.7 Hz, $1 \mathrm{H}), 2.35$ (dt, $J=21.0,5.7 \mathrm{~Hz}, 1 \mathrm{H}), 2.28-2.14(\mathrm{~m}, 1 \mathrm{H}), 2.08(\mathrm{~s}, 3 \mathrm{H}), 2.01(\mathrm{dd}, J=$ 14.0, $6.7 \mathrm{~Hz}, 1 \mathrm{H}), 1.89$ (ddd, $J=13.1,8.8,2.0 \mathrm{~Hz}, 1 \mathrm{H}), 1.56$ (dd, $J=16.4,3.5 \mathrm{~Hz}, 1 \mathrm{H}), 1.43$ (s, $3 \mathrm{H}), 1.39$ (s, $3 \mathrm{H}), 1.42-1.34 \mathrm{ppm}(\mathrm{m}, 1 \mathrm{H}) ;{ }^{13} \mathrm{C} \mathrm{NMR}\left(100 \mathrm{MHz}, \mathrm{CDCl}_{3}\right) \delta=200.2,198.8,170.7$, 138.9 (2C), 135.2, 128.1, 98.3, 96.3, 72.3, 67.0, 66.1, 55.4, 52.0, 51.2, 47.8, 43.2, 42.0, 35.6, 33.2, 25.4, 23.2, 22.6, 22.4, 21.8, 21.6 ppm; IR (film) $v_{\max }=2937,2869,1739,1682,1451,1381,1260$, 1228, 1222, 1106, 1013, 837, $749 \mathrm{~cm}^{-1}$; HRMS (ESI): calcd for $\mathrm{C}_{26} \mathrm{H}_{34} \mathrm{NaO}_{8}{ }^{+}[\mathrm{M}+\mathrm{Na}]^{+}$ 497.2146, found 497.2143. 


\section{Preparation of alkene 23:}

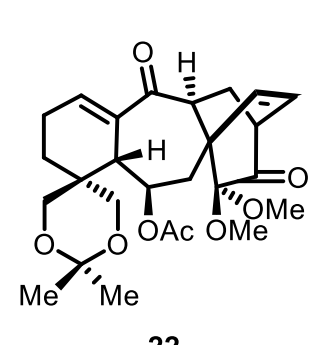

22

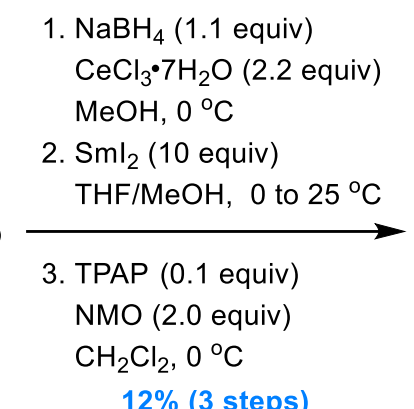

$12 \%$ (3 steps)

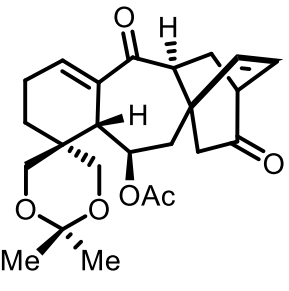

23

To a stirred solution of tetracyclic diene $22(0.15 \mathrm{~g}, 0.32 \mathrm{mmol}, 1.0$ equiv) in $\mathrm{MeOH}(20 \mathrm{~mL})$ at $0{ }^{\circ} \mathrm{C}$ were sequentially added $\mathrm{CeCl}_{3} \cdot 7 \mathrm{H}_{2} \mathrm{O}\left(0.26 \mathrm{~g}, 0.70 \mathrm{mmol}, 2.2\right.$ equiv) and $\mathrm{NaBH}_{4}(13 \mathrm{mg}$, 0.35 mmol, 1.1 equiv). The resulting mixture was stirred at $0{ }^{\circ} \mathrm{C}$ for $0.5 \mathrm{~h}$ before it was added $\mathrm{NH}_{4} \mathrm{Cl}$ (10 mL, sat. aq.). The layers were separated, and the aqueous layer was extracted with EtOAc $(3 \times 5 \mathrm{~mL})$. The combined organic layers were washed with brine $(5 \mathrm{~mL})$, dried $\left(\mathrm{Na}_{2} \mathrm{SO}_{4}\right)$ and concentrated in vacuo to afford the crude alcohol as a pale yellow oil, which was used without further purification.

To a stirred solution of the alcohol (crude, obtained above) in $\mathrm{THF} / \mathrm{MeOH}(10: 1,10 \mathrm{~mL})$ at 0 ${ }^{\circ} \mathrm{C}$ was added $\mathrm{SmI}_{2}(32 \mathrm{~mL}, 0.1 \mathrm{M}$ in THF, $3.2 \mathrm{mmol}, 10$ equiv). The resulting mixture was warmed to $25{ }^{\circ} \mathrm{C}$ and stirred for $1 \mathrm{~h}$ before it was added $\mathrm{NaHCO}_{3}(20 \mathrm{~mL}$, sat. aq.). The layers were separated, and the aqueous layer was extracted with EtOAc $(3 \times 10 \mathrm{~mL})$. The combined organic layers were washed with brine $(10 \mathrm{~mL})$, dried $\left(\mathrm{Na}_{2} \mathrm{SO}_{4}\right)$ and concentrated in vacuo to afford the crude ketone as a pale yellow oil, which was used without further purification.

To a stirred solution of the ketone (crude, obtained above) in $\mathrm{CH}_{2} \mathrm{Cl}_{2}(20 \mathrm{~mL})$ at $0{ }^{\circ} \mathrm{C}$ were sequentially added TPAP (11 mg, $30 \mu \mathrm{mol}, 0.1$ equiv) and NMO (75 mg, 0.64 mmol, 2.0 equiv). The resulting mixture was stirred at $0{ }^{\circ} \mathrm{C}$ for $1 \mathrm{~h}$ before it was added $\mathrm{Na}_{2} \mathrm{~S}_{2} \mathrm{O}_{3}(10 \mathrm{~mL}$, sat. aq.). The layers were separated, and the aqueous layer was extracted with $\mathrm{CH}_{2} \mathrm{Cl}_{2}(3 \times 5 \mathrm{~mL})$. The combined organic layers were dried $\left(\mathrm{Na}_{2} \mathrm{SO}_{4}\right)$ and concentrated in vacuo. Flash column 
chromatography (silica gel, hexanes:EtOAc 2:1) afforded diene 23 (16 mg, 12\% over three steps) as a white amorphous solid. 23: $R_{\mathrm{f}}=0.45$ (silica gel, hexanes:EtOAc 2:1); $[\alpha]_{\mathrm{D}}{ }^{25}=-56.3(\mathrm{c}=$ $\left.0.40, \mathrm{CHCl}_{3}\right) ;{ }^{1} \mathrm{H} \mathrm{NMR}\left(400 \mathrm{MHz}, \mathrm{CDCl}_{3}\right) \delta=6.56(\mathrm{~d}, J=8.3 \mathrm{~Hz}, 1 \mathrm{H}), 6.32(\mathrm{dd}, J=8.3,6.5 \mathrm{~Hz}$, $1 \mathrm{H}), 5.94(\mathrm{t}, J=3.7 \mathrm{~Hz}, 1 \mathrm{H}), 5.35(\mathrm{dt}, J=7.9,3.8 \mathrm{~Hz}, 1 \mathrm{H}), 3.78(\mathrm{~d}, J=11.8 \mathrm{~Hz}, 1 \mathrm{H}), 3.66-$ $3.54(\mathrm{~m}, 2 \mathrm{H}), 3.36(\mathrm{dd}, J=11.8,1.4 \mathrm{~Hz}, 1 \mathrm{H}), 3.27-3.18(\mathrm{~m}, 1 \mathrm{H}), 2.65(\mathrm{dd}, J=9.3,5.1 \mathrm{~Hz}, 1 \mathrm{H})$, 2.46-2.35 (m, $2 \mathrm{H}), 2.26(\mathrm{~d}, J=3.8 \mathrm{~Hz}, 2 \mathrm{H}), 2.24-2.12(\mathrm{~m}, 3 \mathrm{H}), 2.09$ (s, $3 \mathrm{H}), 2.05-1.97$ (m, 1 $\mathrm{H}), 1.96-1.85(\mathrm{~m}, 2 \mathrm{H}), 1.51-1.41(\mathrm{~m}, 1 \mathrm{H}), 1.39 \mathrm{ppm}(\mathrm{s}, 6 \mathrm{H}) ;{ }^{13} \mathrm{C} \mathrm{NMR}\left(100 \mathrm{MHz}, \mathrm{CDCl}_{3}\right) \delta=$ 210.5, 205.3, 170.2, 139.4, 134.6, 130.7, 130.3, 98.3, 72.1, 67.2, 66.0, 55.7, 48.5 (2C), 42.2, 41.6, 40.5, 35.0, 25.2, 24.7, 22.6, 22.5, 21.8 ppm (2C); IR (film) $v_{\max }=2947,2857,1739,1673,1463$, 1365, 1249, 1153, 1022, 845, $738 \mathrm{~cm}^{-1}$; HRMS (ESI): calcd for $\mathrm{C}_{24} \mathrm{H}_{30} \mathrm{NaO}_{6}{ }^{+}[\mathrm{M}+\mathrm{Na}]^{+} 437.1935$, found 437.1937 .

Table S2. Optimization of the HAT-Initiated Transannular Redox Radical Cyclization of $22^{a}$

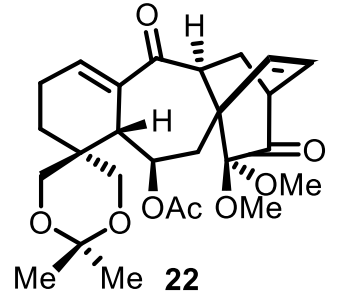

conditions

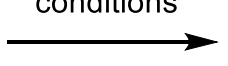

Me 22

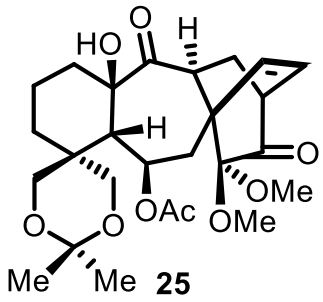

Me 25

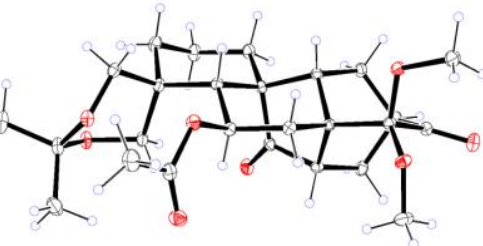

X-ray of 27 [CCDC 2086700]

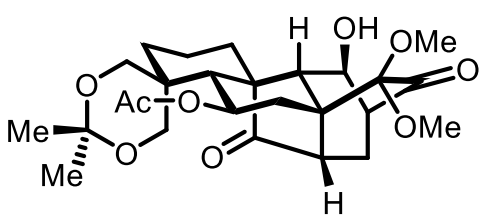

26

$+$

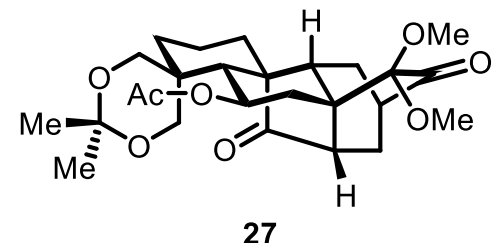

27

\begin{tabular}{lcrcc}
\hline entry & conditions & \multicolumn{3}{c}{ yield $(\boldsymbol{\%})^{b}$} \\
\cline { 3 - 5 } & & $\mathbf{2 5}$ & $\mathbf{2 6}$ & $\mathbf{2 7}$ \\
\hline $1^{c}$ & $\mathrm{Fe}(\mathrm{Pc}), \mathrm{NaBH}_{4}, \mathrm{O}_{2}, \mathrm{EtOH}$ & 0 & 0 & 0 \\
$2^{d, e}$ & $\mathrm{Fe}(\mathrm{acac})_{3}, \mathrm{PhSiH}_{3}$ or $\mathrm{Ph}(i-\mathrm{PrO}) \mathrm{SiH}_{2}, \mathrm{O}_{2}$ & 0 & 0 & 0 \\
& $\mathrm{EtOH} /\left(\mathrm{CH}_{2} \mathrm{OH}\right)_{2}(5: 1 v / v)$ & & \\
\hline
\end{tabular}




\begin{tabular}{|c|c|c|c|c|}
\hline $3^{e}$ & $\begin{array}{c}\mathrm{Fe}(\mathrm{dibm})_{3}, \mathrm{PhSiH}_{3} \text { or } \mathrm{Ph}(i-\mathrm{PrO}) \mathrm{SiH}_{2}, \mathrm{O}_{2} \\
\mathrm{EtOH} / \mathrm{CF}_{3} \mathrm{Ph}(1: 10 v / v)\end{array}$ & 0 & 0 & 0 \\
\hline $4^{e}$ & $\begin{array}{c}\mathrm{Fe}(\mathrm{dpm})_{3}, \mathrm{PhSiH}_{3} \text { or } \mathrm{Ph}(i-\mathrm{PrO}) \mathrm{SiH}_{2}, \mathrm{O}_{2} \\
\mathrm{DCE} / \mathrm{EtOH}(1: 4 v / v)\end{array}$ & 0 & 0 & 0 \\
\hline $5^{e}$ & $\mathrm{Mn}(\mathrm{dpm})_{3}, \mathrm{PhSiH}_{3}$ or $\mathrm{Ph}(i-\mathrm{PrO}) \mathrm{SiH}_{2}, \mathrm{O}_{2}, i-\mathrm{PrOH}$ & 0 & 0 & 0 \\
\hline $6^{e, f}$ & $\mathrm{Mn}(\mathrm{dpm})_{3}, \mathrm{Ph}(i-\mathrm{PrO}) \mathrm{SiH}_{2}, \mathrm{TBHP}, \mathrm{O}_{2}$, hexanes & 0 & 0 & 0 \\
\hline 7 & $\mathrm{Mn}(\mathrm{acac})_{2}, \mathrm{PhSiH}_{3}, \mathrm{PPh}_{3}, \mathrm{O}_{2}, \mathrm{EtOH}$ & 89 & 0 & 0 \\
\hline 8 & $\mathrm{Co}(\mathrm{acac})_{2}, \mathrm{PhSiH}_{3}, \mathrm{O}_{2}, i-\mathrm{PrOH}$ & $<5$ & 75 & 12 \\
\hline 9 & $\mathrm{Co}(\mathrm{tfa})_{2}, \mathrm{PhSiH}_{3}, \mathrm{O}_{2}, i-\mathrm{PrOH}$ & $<5$ & 62 & 10 \\
\hline 10 & $\mathbf{C 1}, \mathrm{PhSiH}_{3}, \mathrm{O}_{2}, i-\mathrm{PrOH}$ & $<5$ & 39 & $<5$ \\
\hline 11 & $\mathbf{C 2}, \mathrm{PhSiH}_{3}, \mathrm{O}_{2}, \mathrm{EtOH}$ & $<5$ & 42 & $<5$ \\
\hline 12 & $\mathbf{C 3}, \mathrm{PhSiH}_{3}, \mathrm{O}_{2}, i-\mathrm{PrOH}$ & $<5$ & 53 & $<5$ \\
\hline 13 & $\mathbf{C 4}, \mathrm{PhSiH}_{3}, \mathrm{O}_{2}, \mathrm{EtOH}$ & $<5$ & 36 & 0 \\
\hline 14 & $\mathbf{C 5}, \mathrm{PhSiH}_{3}, \mathrm{O}_{2}, i-\mathrm{PrOH}$ & $<5$ & 61 & $<5$ \\
\hline 15 & C6, $\mathrm{PhSiH}_{3}, \mathrm{O}_{2}, i-\mathrm{PrOH}$ & 0 & 28 & 0 \\
\hline 16 & C7, $\mathrm{PhSiH}_{3}, \mathrm{O}_{2}, i-\mathrm{PrOH}$ & $<5$ & 57 & $<5$ \\
\hline 17 & $\mathbf{C 8}, \mathrm{PhSiH}_{3}, \mathrm{O}_{2}, i-\mathrm{PrOH}$ & $<5$ & 45 & $<5$ \\
\hline 18 & C9, $\mathrm{PhSiH}_{3}, \mathrm{O}_{2}, i-\mathrm{PrOH}$ & $<5$ & 58 & $<5$ \\
\hline 19 & C10, $\mathrm{PhSiH}_{3}, \mathrm{O}_{2}, \mathrm{EtOH}$ & $<5$ & 23 & $<5$ \\
\hline 20 & C11, $\mathrm{PhSiH}_{3}, \mathrm{O}_{2}, i$-PrOH & $<5$ & 69 & $<5$ \\
\hline 21 & $\mathbf{C 1 2}, \mathrm{PhSiH}_{3}, \mathrm{O}_{2}, i-\mathrm{PrOH}$ & 0 & 16 & 0 \\
\hline 22 & $\mathbf{C 1 3}, \mathrm{PhSiH}_{3}, \mathrm{O}_{2}, \mathrm{EtOH}$ & $<5$ & 27 & 0 \\
\hline 23 & $\mathbf{C 1 4}, \mathrm{PhSiH}_{3}, \mathrm{O}_{2}, i-\mathrm{PrOH}$ & 0 & 18 & 0 \\
\hline 24 & C15, $\mathrm{PhSiH}_{3}, \mathrm{O}_{2}, i-\mathrm{PrOH}$ & $<5$ & 31 & 0 \\
\hline 25 & C16, $\mathrm{PhSiH}_{3}, \mathrm{O}_{2}, i-\mathrm{PrOH}$ & $<5$ & 12 & 0 \\
\hline 26 & $\mathrm{Co}(\mathrm{acac})_{2}, \mathrm{PhSiH}_{3}, \mathrm{O}_{2}, \mathrm{HFIP}$ & $<5$ & 62 & 16 \\
\hline 27 & $\mathrm{Co}(\mathrm{acac})_{2}, \mathrm{PhSiH}_{3}, \mathrm{O}_{2}, 1,4$-dioxane & $<5$ & 71 & 13 \\
\hline 28 & $\mathrm{Co}(\mathrm{acac})_{2}, \mathrm{PhSiH}_{3}, \mathrm{O}_{2}, \mathrm{DCE}$ & $<5$ & 56 & 8 \\
\hline 29 & $\mathrm{Co}(\mathrm{acac})_{2}, \mathrm{Ph}(i-\mathrm{PrO}) \mathrm{SiH}_{2}, \mathrm{O}_{2}, i-\mathrm{PrOH}$ & $<5$ & 70 & 15 \\
\hline 30 & $\mathrm{Co}(\mathrm{acac})_{2}, \mathrm{Ph}_{2} \mathrm{SiH}_{2}, \mathrm{O}_{2}, i-\mathrm{PrOH}$ & $<5$ & 69 & 11 \\
\hline 31 & $\mathrm{Co}(\mathrm{acac})_{2}, \mathrm{TMDSO}, \mathrm{O}_{2}, i-\mathrm{PrOH}$ & $<5$ & 77 & 7 \\
\hline 32 & $\mathrm{Co}(\mathrm{acac})_{2}, \mathrm{TMDSO}, \mathrm{O}_{2}, 4 \AA \mathrm{MS}, i-\mathrm{PrOH}$ & $<5$ & $86(83)^{g}$ & $<5$ \\
\hline 33 & $\begin{array}{c}\mathrm{Co}(\mathrm{acac})_{2}, \mathrm{TMDSO}, \mathrm{O}_{2} / \mathrm{Ar}(1: 10 v / v, 1 \mathrm{~atm}) \\
4 \AA \mathrm{MS}, i-\mathrm{PrOH}\end{array}$ & 40 & 15 & 0 \\
\hline 34 & $\mathrm{Co}(\mathrm{acac})_{2}, \mathrm{TMDSO}, \mathrm{O}_{2}(1 \mathrm{~atm}), 4 \AA \mathrm{MS}, i-\mathrm{PrOH}$ & 63 & 6 & 0 \\
\hline
\end{tabular}




\begin{tabular}{lcllc}
\hline $35^{e}$ & $\mathrm{Fe}(\mathrm{acac})_{3}, \mathrm{PhSiH} \mathrm{H}_{3}$ or TMDSO, $4 \AA \mathrm{MS}$ & 0 & 0 & $<5$ \\
$\mathrm{EtOH} /\left(\mathrm{CH}_{2} \mathrm{OH}\right)_{2}(5: 1$ v/v) & & & \\
$36^{e, f}$ & $\mathrm{Mn}(\mathrm{dpm})_{3}, \mathrm{Ph}(i-\mathrm{PrO}) \mathrm{SiH}_{2}$ or TMDSO, TBHP & 0 & 0 & $<5$ \\
& $4 \AA \mathrm{MS}$, hexanes & & & \\
$37^{f}$ & $\mathbf{C 1 3}, \mathrm{TMDSO}, \mathrm{TBHP}, 4 \AA \mathrm{MS}, i-\mathrm{PrOH}$ & 0 & 0 & 56 \\
$38^{f}$ & $\mathbf{C 1 4}, \mathrm{TMDSO}, \mathrm{TBHP}, 4 \AA \mathrm{MS}, i-\mathrm{PrOH}$ & 0 & 0 & 33 \\
$39^{f}$ & $\mathbf{C 1 5}, \mathrm{TMDSO}, \mathrm{TBHP}, 4 \AA \mathrm{MS}, i-\mathrm{PrOH}$ & 0 & 0 & 65 \\
$40^{f, h}$ & $\mathbf{C 1 6}, \mathrm{TMDSO}, \mathrm{TBHP}, 4 \AA \mathrm{MS}, i-\mathrm{PrOH}$ & 0 & 0 & 0 \\
\hline
\end{tabular}

${ }^{a}$ Reaction conditions: $22(0.05 \mathrm{mmol}),[\mathrm{M}](20 \mathrm{~mol} \%),[\mathrm{B}] /[\mathrm{Si}]$ (2.0 equiv), $\mathrm{O}_{2}$ (2.2 equiv), solvent $(4 \mathrm{~mL}), 25{ }^{\circ} \mathrm{C}$. ${ }^{b}$ Isolated yields. ${ }^{c}$ Unidentified mixture. ${ }^{d}$ Performed at $60 \quad{ }^{\circ} \mathrm{C}$. ${ }^{e}$ Decomposition. ${ }^{\text {T }}$ TBHP (2.0 equiv). ${ }^{g}$ Gram scale. ${ }^{h}$ Recovery of 22.

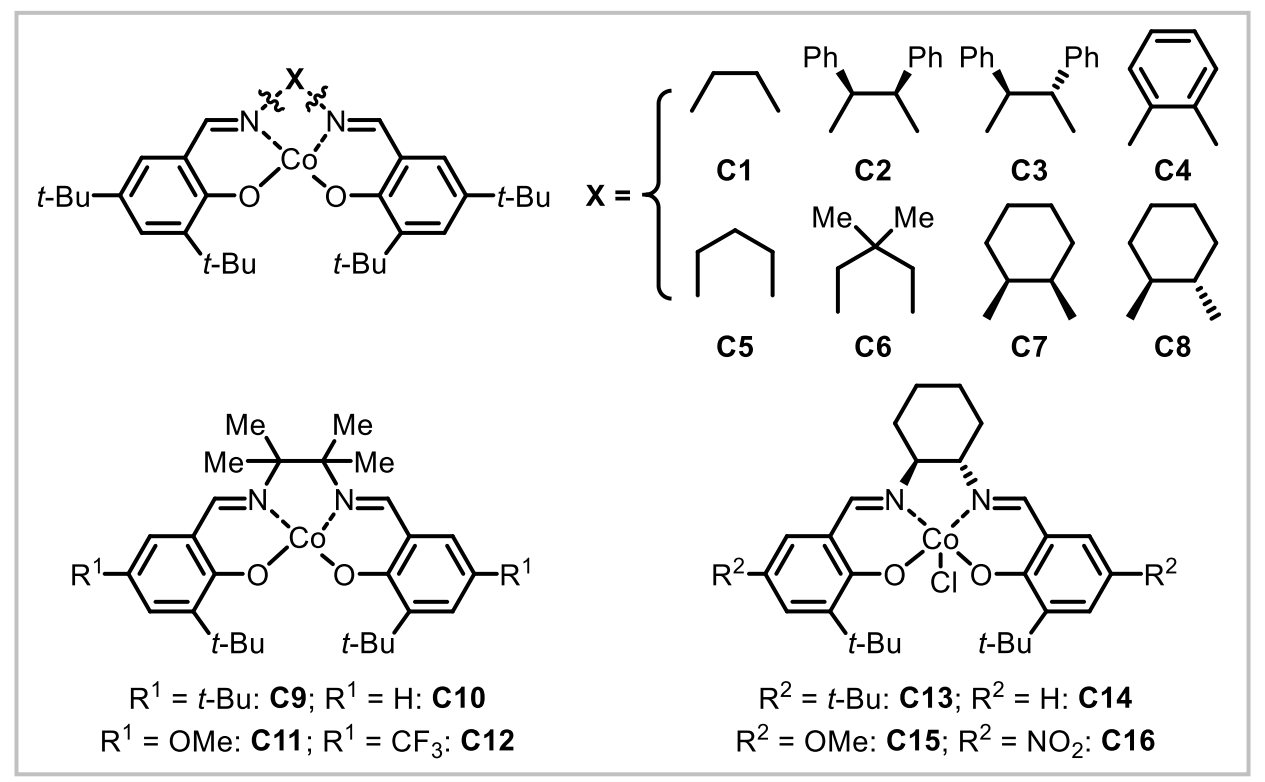

\section{Preparation of alcohol 25 (entry 7, Table S2):}

To a stirred solution of tetracyclic diene $22(24 \mathrm{mg}, 50 \mu \mathrm{mol}, 1.0$ equiv) in EtOH (4 mL) at $25^{\circ} \mathrm{C}$ was injected $\mathrm{O}_{2}(2.5 \mathrm{~mL}, 0.11 \mathrm{mmol}, 2.2$ equiv $)$ by the means of a graduated syringe into the solution. The resulting mixture was stirred for $0.5 \mathrm{~h}$ before it was sequentially added $\mathrm{Mn}(\text { acac })_{2}\left(2.5 \mathrm{mg}, 10 \mu \mathrm{mol}, 0.2\right.$ equiv), $\mathrm{Ph}_{3} \mathrm{P}\left(26 \mathrm{mg}, 0.10 \mathrm{mmol}, 2.0\right.$ equiv) and $\mathrm{PhSiH}_{3}(12 \mu \mathrm{L}$, $0.10 \mathrm{mmol}, 2.0$ equiv). The reaction was stirred for an additional $4 \mathrm{~h}$ before it was concentrated in vасио. Flash column chromatography (silica gel, hexanes:EtOAc 2:1) afforded alcohol 25 (22 mg, 
$89 \%$ ) as a colorless oil. 25: $R_{\mathrm{f}}=0.40$ (silica gel, hexanes:EtOAc $\left.2: 1\right) ;[\alpha]_{\mathrm{D}}{ }^{25}=-51.6(\mathrm{c}=0.50$, $\left.\mathrm{CHCl}_{3}\right) ;{ }^{1} \mathrm{H} \mathrm{NMR}\left(400 \mathrm{MHz}, \mathrm{CDCl}_{3}\right) \delta=6.23(\mathrm{dd}, J=8.3,6.7 \mathrm{~Hz}, 1 \mathrm{H}), 6.09(\mathrm{~d}, J=8.2 \mathrm{~Hz}, 1 \mathrm{H})$, $5.62-5.53(\mathrm{~m}, 1 \mathrm{H}), 4.11(\mathrm{~d}, J=12.0 \mathrm{~Hz}, 1 \mathrm{H}), 4.00(\mathrm{~d}, J=12.1 \mathrm{~Hz}, 2 \mathrm{H}), 3.86(\mathrm{~d}, J=11.9 \mathrm{~Hz}, 1$ H), $3.70(\mathrm{dd}, J=8.9,4.1 \mathrm{~Hz}, 1 \mathrm{H}), 3.47(\mathrm{~s}, 3 \mathrm{H}), 3.45(\mathrm{~d}, J=11.9 \mathrm{~Hz}, 1 \mathrm{H}), 3.29-3.24(\mathrm{~m}, 1 \mathrm{H})$, $3.22(\mathrm{~s}, 3 \mathrm{H}), 2.66(\mathrm{dd}, J=16.4,6.3 \mathrm{~Hz}, 1 \mathrm{H}), 2.45(\mathrm{dd}, J=16.3,3.0 \mathrm{~Hz}, 1 \mathrm{H}), 2.34$ (dt, $J=13.2$, $3.9 \mathrm{~Hz}, 1 \mathrm{H}), 2.09-1.98(\mathrm{~m}, 1 \mathrm{H}), 2.02(\mathrm{~s}, 3 \mathrm{H}), 1.93-1.76(\mathrm{~m}, 4 \mathrm{H}), 1.65-1.58(\mathrm{~m}, 2 \mathrm{H}), 1.56-$ 1.47 (m, $1 \mathrm{H}), 1.40$ (s, $3 \mathrm{H}), 1.37 \mathrm{ppm}(\mathrm{s}, 3 \mathrm{H}) ;{ }^{13} \mathrm{C} \mathrm{NMR}\left(100 \mathrm{MHz}, \mathrm{CDCl}_{3}\right) \delta=212.8,201.4$, $169.4,135.5,127.6,98.2,96.0,80.4,70.5,68.5,67.3,56.1,52.3,50.8,49.8,47.9,44.5,38.2,34.0$, 31.6, 27.4, 25.7, 25.4, 22.3, 21.6, 17.0 ppm; IR (film) $v_{\max }=2953,2846,1734,1685,1471,1373$, 1236, 1220, 1153, 1096, $746 \mathrm{~cm}^{-1}$; HRMS (ESI): calcd for $\mathrm{C}_{26} \mathrm{H}_{36} \mathrm{NaO}_{9}^{+}[\mathrm{M}+\mathrm{Na}]^{+} 515.2252$, found 515.2254.

\section{Preparation of pentacyclic alcohol 26 (entry 32, Table S2):}

To a stirred solution of tetracyclic diene $22(1.8 \mathrm{~g}, 3.8 \mathrm{mmol}, 1.0$ equiv) and $4 \AA \mathrm{MS}(7.2 \mathrm{~g})$ in rigorously degassed $i$-PrOH $(300 \mathrm{~mL})$ at $25{ }^{\circ} \mathrm{C}$ was injected $\mathrm{O}_{2}(0.19 \mathrm{~L}, 8.4 \mathrm{mmol}, 2.2$ equiv) by the means of a graduated syringe into the solution. The reaction was stirred for $0.5 \mathrm{~h}$ before it was sequentially added $\mathrm{Co}(\mathrm{acac})_{2}(0.20 \mathrm{~g}, 0.76 \mathrm{mmol}, 0.2$ equiv) and TMDSO (1.3 mL, $7.6 \mathrm{mmol}, 2.0$ equiv). The resulting mixture was stirred for an additional $72 \mathrm{~h}$ before it was concentrated in vacuo. Flash column chromatography (silica gel, hexanes:EtOAc 2:1) afforded pentacyclic alcohol 26 (1.6 g, 83\%) as a white solid. 26: CCDC 2086699; m.p. $114-115^{\circ} \mathrm{C}\left(\mathrm{CH}_{2} \mathrm{Cl}_{2} / \mathrm{MeOH}\right)$; $R_{\mathrm{f}}=0.35$ (silica gel, hexanes:EtOAc 2:1); $[\alpha]_{\mathrm{D}}{ }^{25}=-62.4\left(\mathrm{c}=0.50, \mathrm{CHCl}_{3}\right) ; 97: 3$ er [recrystallization $\left(\mathrm{CH}_{2} \mathrm{Cl}_{2} / \mathrm{MeOH} /\right.$ hexanes $\left.1: 1: 5,92 \%\right)$; Daicel Chiralpak AD-H $(0.46 \mathrm{~cm} \times 25$ $\mathrm{cm}$ ), $n$-hexane/ 2-propanol $=90 / 10, \mathrm{v}=1.0 \mathrm{~mL} \cdot \min ^{-1}, \lambda=210 \mathrm{~nm}, \mathrm{t}$ (major) $=11.87 \mathrm{~min}, \mathrm{t}($ minor $)$ $=9.00 \mathrm{~min}] .{ }^{1} \mathrm{H} \mathrm{NMR}\left(400 \mathrm{MHz}, \mathrm{CDCl}_{3}\right) \delta=5.12(\mathrm{td}, J=11.3,6.1 \mathrm{~Hz}, 1 \mathrm{H}), 4.20(\mathrm{t}, J=4.6 \mathrm{~Hz}, 1$ 
H), $3.92(\mathrm{~d}, J=11.6 \mathrm{~Hz}, 1 \mathrm{H}), 3.78(\mathrm{~d}, J=11.9 \mathrm{~Hz}, 1 \mathrm{H}), 3.52(\mathrm{~s}, 3 \mathrm{H}), 3.51(\mathrm{~d}, J=11.5 \mathrm{~Hz}, 1 \mathrm{H})$, $3.36(\mathrm{~s}, 3 \mathrm{H}), 3.14(\mathrm{~d}, J=11.5 \mathrm{~Hz}, 1 \mathrm{H}), 2.56(\mathrm{t}, J=4.0 \mathrm{~Hz}, 1 \mathrm{H}), 2.51(\mathrm{dd}, J=10.5,3.6 \mathrm{~Hz}, 1 \mathrm{H})$, 2.46-2.36 (m, $2 \mathrm{H}), 2.36-2.28(\mathrm{~m}, 1 \mathrm{H}), 2.20(\mathrm{dd}, J=14.5,11.2 \mathrm{~Hz}, 1 \mathrm{H}), 2.15-2.07(\mathrm{~m}, 1 \mathrm{H})$, 2.07-1.98 (m, $2 \mathrm{H}), 2.04(\mathrm{~s}, 3 \mathrm{H}), 1.88(\mathrm{dd}, J=13.1,10.8 \mathrm{~Hz}, 1 \mathrm{H}), 1.78(\mathrm{~d}, J=12.0 \mathrm{~Hz}, 1 \mathrm{H})$, $1.70(\mathrm{dd}, J=15.1,4.6 \mathrm{~Hz}, 1 \mathrm{H}), 1.64-1.55(\mathrm{~m}, 1 \mathrm{H}), 1.35(\mathrm{td}, J=13.4,4.8 \mathrm{~Hz}, 1 \mathrm{H}), 1.27$ (s, $3 \mathrm{H})$, $1.22(\mathrm{~s}, 3 \mathrm{H}), 0.94 \mathrm{ppm}(\mathrm{td}, J=13.5,3.0 \mathrm{~Hz}, 1 \mathrm{H}) ;{ }^{13} \mathrm{C} \mathrm{NMR}\left(100 \mathrm{MHz}, \mathrm{CDCl}_{3}\right) \delta=222.8,204.8$, $170.4,99.5,95.7,70.7,69.7,68.4,59.0,58.8,55.6,54.3,52.6,52.4,50.3,50.1,49.1,40.0,33.2$, 32.5, 29.9, 26.3, 25.1, 22.4, 21.7, 18.0 ppm; IR (film) $v_{\max }=3447,2945,2841,1735,1457,1370$, 1228, 1131, 1087, $741 \mathrm{~cm}^{-1}$; HRMS (ESI): calcd for $\mathrm{C}_{26} \mathrm{H}_{36} \mathrm{NaO}_{9}{ }^{+}[\mathrm{M}+\mathrm{Na}]^{+}$515.2252, found 515.2263 .

\section{Preparation of pentacycle 27 (entry 39, Table S2):}

To a stirred solution of tetracyclic diene 22 (24 mg, $50 \mu \mathrm{mol}, 1.0$ equiv) and $4 \AA \mathrm{MS}(0.10 \mathrm{~g})$ in rigorously degassed $i$ - $\mathrm{PrOH}(4 \mathrm{~mL})$ at $25{ }^{\circ} \mathrm{C}$ were sequentially added $\mathrm{Co}(\mathrm{Salen})$ complex $\mathbf{C 1 5}^{4}$ (5.9 mg, $10 \mu \mathrm{mol}, 0.2$ equiv), TBHP ( $18 \mu \mathrm{L}, 5.5 \mathrm{M}$ in decane, $0.10 \mathrm{mmol}, 2.0$ equiv) and TMDSO (18 $\mu \mathrm{L}, 0.10 \mathrm{mmol}, 2.0$ equiv). The reaction was stirred for $2 \mathrm{~h}$ before it was concentrated in vacuo. Flash column chromatography (silica gel, hexanes:EtOAc 2:1) afforded pentacycle 27 (15 mg, 65\%) as a white solid. 27: CCDC 2086700; m.p. 201-203 ${ }^{\circ} \mathrm{C}\left(\mathrm{CH}_{2} \mathrm{Cl}_{2} /\right.$ hexanes $) ; R_{\mathrm{f}}=0.40$ (silica gel, hexanes:EtOAc 3:1); $[\alpha]_{\mathrm{D}}{ }^{25}=-94.7\left(\mathrm{c}=0.40, \mathrm{CHCl}_{3}\right) ;{ }^{1} \mathrm{H} \mathrm{NMR}\left(400 \mathrm{MHz}, \mathrm{CDCl}_{3}\right) \delta$ $=5.10(\mathrm{td}, J=11.3,6.0 \mathrm{~Hz}, 1 \mathrm{H}), 3.89(\mathrm{~d}, J=11.2 \mathrm{~Hz}, 1 \mathrm{H}), 3.78(\mathrm{~d}, J=11.9 \mathrm{~Hz}, 1 \mathrm{H}), 3.51(\mathrm{~d}, J$ $=12.0 \mathrm{~Hz}, 1 \mathrm{H}), 3.46(\mathrm{~s}, 3 \mathrm{H}), 3.34(\mathrm{~s}, 3 \mathrm{H}), 3.11(\mathrm{~d}, J=11.4 \mathrm{~Hz}, 1 \mathrm{H}), 2.48(\mathrm{dd}, J=10.7,3.5 \mathrm{~Hz}$, $1 \mathrm{H}), 2.38(\mathrm{dd}, J=13.0,6.1 \mathrm{~Hz}, 1 \mathrm{H}), 2.34-2.24(\mathrm{~m}, 2 \mathrm{H}), 2.21-1.97(\mathrm{~m}, 5 \mathrm{H}), 2.02(\mathrm{~s}, 3 \mathrm{H})$, 1.96-1.88 (m, $1 \mathrm{H}), 1.87-1.75(\mathrm{~m}, 2 \mathrm{H}), 1.67(\mathrm{~d}, J=12.0 \mathrm{~Hz}, 1 \mathrm{H}), 1.60-1.50(\mathrm{~m}, 1 \mathrm{H}), 1.25(\mathrm{~s}, 3$ $\mathrm{H}), 1.20(\mathrm{~s}, 3 \mathrm{H}), 1.09(\mathrm{td}, J=13.4,4.6 \mathrm{~Hz}, 1 \mathrm{H}), 0.87 \mathrm{ppm}(\mathrm{t}, J=13.0 \mathrm{~Hz}, 1 \mathrm{H}) ;{ }^{13} \mathrm{C}$ NMR $(100$ 
$\left.\mathrm{MHz}, \mathrm{CDCl}_{3}\right) \delta=223.8,206.2,170.3,99.4,96.2,70.7,68.5,59.0,55.6,53.3$ (2C), 52.7, 50.5, 49.2, 48.3, 41.3, 39.9, 33.7, 32.7, 30.4, 29.2, 25.1, 24.4, 22.4, 21.6, 18.0 ppm; IR (film) $v_{\max }=$ 2943, 2846, 1736, 1456, 1370, 1240, 1150, 1087, 1035, $736 \mathrm{~cm}^{-1}$; HRMS (ESI): calcd for $\mathrm{C}_{26} \mathrm{H}_{36} \mathrm{NaO}_{8}{ }^{+}[\mathrm{M}+\mathrm{Na}]^{+}$499.2302, found 499.2303.

\section{Preparation of diketone S-4:}

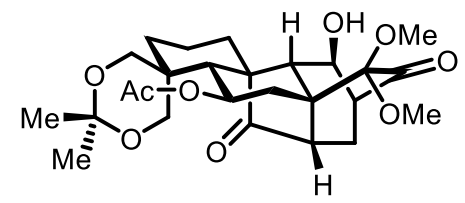

26

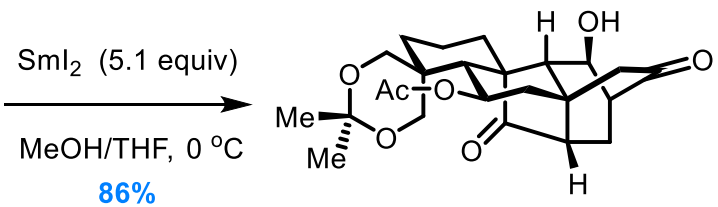

S-4

To a stirred solution of pentacyclic alcohol 26 (2.0 g, $4.1 \mathrm{mmol}, 1.0$ equiv) in THF/MeOH $(10: 1,20 \mathrm{~mL})$ at $0{ }^{\circ} \mathrm{C}$ was added $\mathrm{SmI}_{2}(210 \mathrm{~mL}, 0.1 \mathrm{M}$ in THF, $21 \mathrm{mmol}, 5.1$ equiv). The resulting mixture was stirred at $0{ }^{\circ} \mathrm{C}$ for $1 \mathrm{~h}$ before it was added $\mathrm{NaHCO}_{3}(100 \mathrm{~mL}$, sat. aq.). The layers were separated, and the aqueous layer was extracted with EtOAc $(3 \times 50 \mathrm{~mL})$. The combined organic layers were washed with brine $(50 \mathrm{~mL})$, dried $\left(\mathrm{Na}_{2} \mathrm{SO}_{4}\right)$ and concentrated in vacuo. Flash column chromatography (silica gel, hexanes:EtOAc 1:1) afforded diketone S-4 (1.5 g, 86\%) as a white amorphous solid. S-4: $R_{\mathrm{f}}=0.25$ (silica gel, hexanes:EtOAc 1:1); $[\alpha]_{\mathrm{D}}^{25}=-53.5(\mathrm{c}=0.17$, $\left.\mathrm{CHCl}_{3}\right) ;{ }^{1} \mathrm{H} \mathrm{NMR}\left(400 \mathrm{MHz}, \mathrm{CDCl}_{3}\right) \delta=5.16(\mathrm{td}, J=11.1,6.1 \mathrm{~Hz}, 1 \mathrm{H}), 4.26(\mathrm{t}, J=3.6 \mathrm{~Hz}, 1 \mathrm{H})$, $3.88(\mathrm{~d}, J=11.6 \mathrm{~Hz}, 1 \mathrm{H}), 3.80(\mathrm{~d}, J=12.0 \mathrm{~Hz}, 1 \mathrm{H}), 3.51(\mathrm{~d}, J=12.0 \mathrm{~Hz}, 1 \mathrm{H}), 3.16(\mathrm{~d}, J=11.6$ $\mathrm{Hz}, 1 \mathrm{H}), 2.57(\mathrm{~d}, J=2.6 \mathrm{~Hz}, 1 \mathrm{H}), 2.49(\mathrm{t}, J=4.5 \mathrm{~Hz}, 1 \mathrm{H}), 2.42-2.26(\mathrm{~m}, 4 \mathrm{H}), 2.22(\mathrm{dd}, J=$ 12.9, 6.1 Hz, 1 H), 2.18-2.05 (m, $3 \mathrm{H}), 2.04$ (s, $3 \mathrm{H}), 1.82(\mathrm{~d}, J=12.0 \mathrm{~Hz}, 1 \mathrm{H}), 1.80-1.72$ (m, 2 H), 1.68-1.59 (m, $2 \mathrm{H}), 1.39$ (td, $J=13.1,4.7 \mathrm{~Hz}, 1 \mathrm{H}), 1.27(\mathrm{~s}, 3 \mathrm{H}), 1.22(\mathrm{~s}, 3 \mathrm{H}), 0.96 \mathrm{ppm}(\mathrm{t}, J$ $=13.0 \mathrm{~Hz}, 1 \mathrm{H}) ;{ }^{13} \mathrm{C} \mathrm{NMR}\left(100 \mathrm{MHz}, \mathrm{CDCl}_{3}\right) \delta=223.1,211.3,170.5,99.6,70.7,69.4,67.9,60.9$, 58.9, 56.0, 52.8, 50.3, 50.0, 42.3, 41.5, 40.2 (2C), 32.7, 29.9, 26.7, 24.8, 22.7, 21.6, $18.1 \mathrm{ppm}$; IR 
(film) $v_{\max }=3378,2932,2841,1726,1367,1251,1226,1084,1031,736 \mathrm{~cm}^{-1}$; HRMS (ESI): calcd for $\mathrm{C}_{24} \mathrm{H}_{32} \mathrm{NaO}_{7}{ }^{+}[\mathrm{M}+\mathrm{Na}]^{+}$455.2040, found 455.2051.

\section{Preparation of triol 28:}

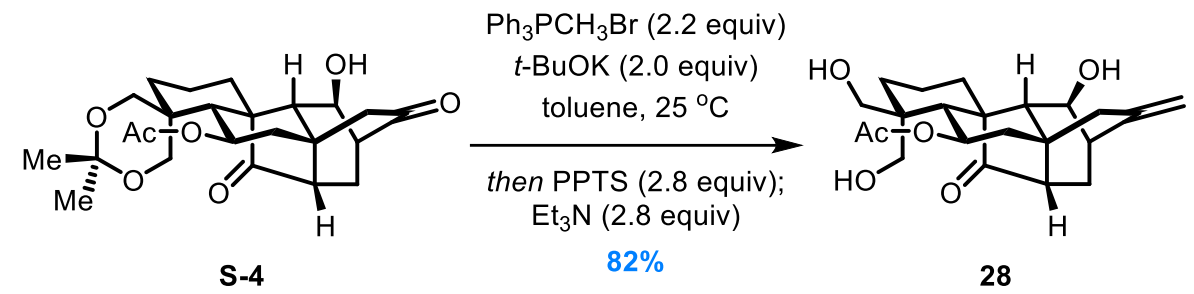

To a stirred solution of $\mathrm{Ph}_{3} \mathrm{PCH}_{3} \mathrm{Br}\left(1.3 \mathrm{~g}, 3.5 \mathrm{mmol}, 2.2\right.$ equiv) in toluene $(15 \mathrm{~mL})$ at $0{ }^{\circ} \mathrm{C}$ was added $t$-BuOK $(3.2 \mathrm{~mL}, 1.0 \mathrm{M}$ in THF, $3.2 \mathrm{mmol}, 2.0$ equiv). The resulting mixture was warmed to $25{ }^{\circ} \mathrm{C}$ and stirred for $0.5 \mathrm{~h}$ before it was added a solution of diketone $\mathbf{S}-4$ ( $0.69 \mathrm{~g}, 1.6$ mmol, 1.0 equiv) in toluene $(30 \mathrm{~mL})$. The reaction was stirred for an additional $2 \mathrm{~h}$ before it was added $\mathrm{MeOH}(30 \mathrm{~mL})$ and PPTS (1.1 g, $4.4 \mathrm{mmol}, 2.8$ equiv). The resulting mixture was stirred for further $2 \mathrm{~h}$ before it was cooled to $0{ }^{\circ} \mathrm{C}$, slowly added $\mathrm{Et}_{3} \mathrm{~N}(0.61 \mathrm{~mL}, 4.4 \mathrm{mmol}, 2.8$ equiv) and concentrated in vacuo. Flash column chromatography (silica gel, EtOAc) afforded triol 28 $(0.51 \mathrm{~g}, 82 \%)$ as a pale yellow oil. Due to its instability, 28 was characterized by the corresponding silyl ether $\mathbf{S - 5}$.

\section{Preparation of silyl ether S-5:}

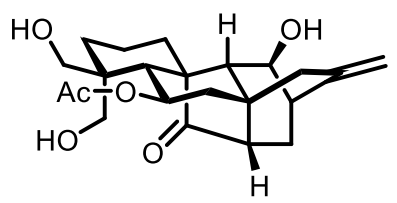

28

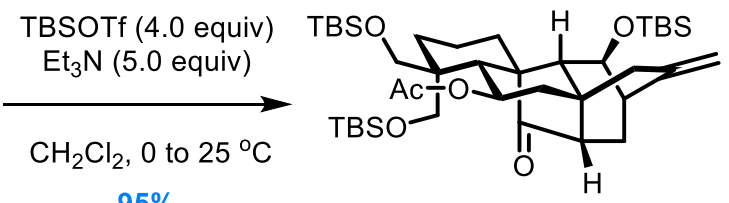

S-5

To a stirred solution of triol 28 (59 mg, $0.15 \mathrm{mmol}, 1.0$ equiv) in $\mathrm{CH}_{2} \mathrm{Cl}_{2}(5 \mathrm{~mL})$ at $0{ }^{\circ} \mathrm{C}$ were sequentially added $\mathrm{Et}_{3} \mathrm{~N}(0.10 \mathrm{~mL}, 0.75 \mathrm{mmol}, 5.0$ equiv) and TBSOTf $(0.14 \mathrm{~mL}, 0.60 \mathrm{mmol}, 4.0$ equiv). The resulting mixture was warmed to $25^{\circ} \mathrm{C}$ and stirred for $1.5 \mathrm{~h}$ before it was added $\mathrm{NaHCO}_{3}(5 \mathrm{~mL}$, sat. aq.). The layers were separated, and the aqueous layer was extracted with 
$\mathrm{CH}_{2} \mathrm{Cl}_{2}(3 \times 5 \mathrm{~mL})$. The combined organic layers were dried $\left(\mathrm{Na}_{2} \mathrm{SO}_{4}\right)$ and concentrated in vacuo. Flash column chromatography (silica gel, hexanes: $\mathrm{CH}_{2} \mathrm{Cl}_{2} 2: 1$ ) afforded silyl ether $\mathbf{S - 5}$ (104 mg, 95\%) as a colorless oil. S-5: $R_{\mathrm{f}}=0.30$ (silica gel, hexanes: $\left.\mathrm{CH}_{2} \mathrm{Cl}_{2} 2: 1\right) ;[\alpha]_{\mathrm{D}}{ }^{25}=-29.2(\mathrm{c}=0.40$, $\left.\mathrm{CHCl}_{3}\right) ;{ }^{1} \mathrm{H} \mathrm{NMR}\left(400 \mathrm{MHz}, \mathrm{CDCl}_{3}\right) \delta=4.79(\mathrm{td}, J=11.3,5.9 \mathrm{~Hz}, 1 \mathrm{H}), 4.68(\mathrm{~d}, J=8.7 \mathrm{~Hz}, 2$ H), $3.88(\mathrm{~d}, J=4.0 \mathrm{~Hz}, 1 \mathrm{H}), 3.82(\mathrm{~d}, J=10.1 \mathrm{~Hz}, 1 \mathrm{H}), 3.60(\mathrm{~d}, J=9.2 \mathrm{~Hz}, 1 \mathrm{H}), 3.45(\mathrm{~d}, J=9.2$ $\mathrm{Hz}, 1 \mathrm{H}), 3.24(\mathrm{~d}, J=10.1 \mathrm{~Hz}, 1 \mathrm{H}), 2.41(\mathrm{~d}, J=12.4 \mathrm{~Hz}, 1 \mathrm{H}), 2.30(\mathrm{~s}, 2 \mathrm{H}), 2.22(\mathrm{t}, J=3.5 \mathrm{~Hz}$, $1 \mathrm{H}), 2.21-2.15$ (dd, $J=11.1,3.3 \mathrm{~Hz}, 1 \mathrm{H}), 2.12-2.06(\mathrm{~m}, 1 \mathrm{H}), 2.06-1.97$ (m, $2 \mathrm{H}), 2.00$ (s, $3 \mathrm{H})$, $1.97-1.90(\mathrm{~m}, 1 \mathrm{H}), 1.81$ (d, $J=13.1 \mathrm{~Hz}, 1 \mathrm{H}), 1.55-1.45$ (m, $3 \mathrm{H}), 1.44-1.30$ (m, $2 \mathrm{H}), 1.27-1.18$ (m, $1 \mathrm{H}), 0.91(\mathrm{~s}, 9 \mathrm{H}), 0.86(\mathrm{~s}, 9 \mathrm{H}), 0.84(\mathrm{~s}, 9 \mathrm{H}), 0.04(\mathrm{~s}, 3 \mathrm{H}), 0.03(\mathrm{~s}, 6 \mathrm{H}), 0.02(\mathrm{~s}, 6 \mathrm{H}),-0.03$ $\operatorname{ppm}(\mathrm{s}, 3 \mathrm{H}) ;{ }^{13} \mathrm{C} \mathrm{NMR}\left(100 \mathrm{MHz}, \mathrm{CDCl}_{3}\right) \delta=227.0,169.7,145.0,107.6,70.6,69.1,68.2,64.3$, 61.2, 52.7, 51.8, 49.3, 43.5, 43.3, 42.5, 38.5, 34.5, 30.6, 30.2, 29.3, 26.1 (6C), 25.8 (3C), 21.8, $18.5,18.4,18.2,18.0,-3.9,-4.3,-5.1,-5.3,-5.4,-5.5 \mathrm{ppm}$; IR (film) $v_{\max }=2928,2856,1737$, 1471, 1250, 1092, 836, $772 \mathrm{~cm}^{-1}$; HRMS (ESI): calcd for $\mathrm{C}_{40} \mathrm{H}_{72} \mathrm{NaO}_{6} \mathrm{Si}_{3}{ }^{+}[\mathrm{M}+\mathrm{Na}]^{+} 755.4529$, found 755.4539 .

\section{Preparation of keto-aldehyde 12:}

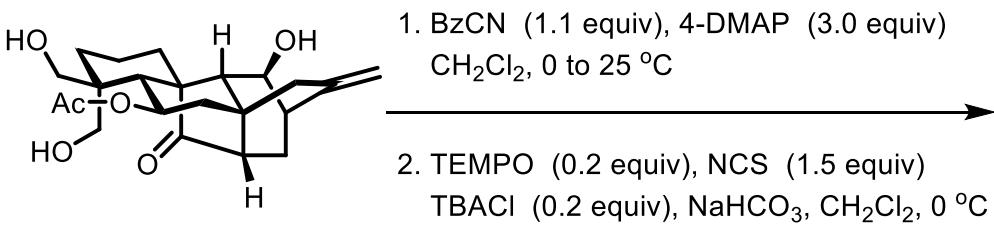

28

$73 \%$ (2 steps)

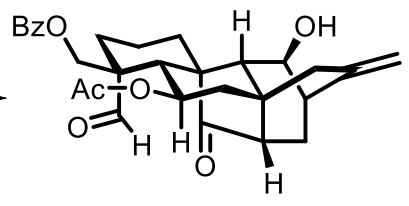

12

To a stirred solution of triol $28\left(0.47 \mathrm{~g}, 1.2 \mathrm{mmol}, 1.0\right.$ equiv) in $\mathrm{CH}_{2} \mathrm{Cl}_{2}(30 \mathrm{~mL})$ at $0{ }^{\circ} \mathrm{C}$ were sequentially added 4-DMAP (0.44 mg, $3.6 \mathrm{mmol}, 3.0$ equiv) and $\mathrm{BzCN}(0.17 \mathrm{~g}, 1.3 \mathrm{mmol}, 1.1$ equiv). The resulting mixture was warmed to $25^{\circ} \mathrm{C}$ and stirred for $1.5 \mathrm{~h}$ before it was added $\mathrm{NaHCO}_{3}(15 \mathrm{~mL}$, sat. aq.). The layers were separated, and the aqueous layer was extracted with $\mathrm{CH}_{2} \mathrm{Cl}_{2}(3 \times 10 \mathrm{~mL})$. The combined organic layers were dried $\left(\mathrm{Na}_{2} \mathrm{SO}_{4}\right)$ and concentrated in 
vacuo to afford the crude benzoate as a pale yellow oil, which should be used immediately due to its instability.

To a stirred solution of the benzoate (crude, obtained above) in $\mathrm{CH}_{2} \mathrm{Cl}_{2}(20 \mathrm{~mL})$ and $\mathrm{NaHCO}_{3}$ (20 mL, $0.5 \mathrm{M}$ aq.) at $0{ }^{\circ} \mathrm{C}$ were sequentially added $\mathrm{TBACl}(67 \mathrm{mg}, 0.24 \mathrm{mmol}, 0.2$ equiv), TEMPO (38 mg, $0.24 \mathrm{mmol}, 0.2$ equiv) and NCS (0.24 g, $1.8 \mathrm{mmol}, 1.5$ equiv). The resulting mixture was stirred at $0{ }^{\circ} \mathrm{C}$ for $3 \mathrm{~h}$ before it was added $\mathrm{Na}_{2} \mathrm{~S}_{2} \mathrm{O}_{3}(20 \mathrm{~mL}$, sat. aq. $)$. The layers were separated, and the aqueous layer was extracted with $\mathrm{CH}_{2} \mathrm{Cl}_{2}(3 \times 10 \mathrm{~mL})$. The combined organic layers were dried $\left(\mathrm{Na}_{2} \mathrm{SO}_{4}\right)$ and concentrated in vacuo. Flash column chromatography (silica gel, hexanes:EtOAc 1:1) afforded keto-aldehyde 12 (0.43 g, 73\% over two steps) as a white amorphous solid. 12: $R_{\mathrm{f}}=0.45$ (silica gel, hexanes:EtOAc 1:1); $[\alpha]_{\mathrm{D}}{ }^{25}=-30.6\left(\mathrm{c}=0.40, \mathrm{CHCl}_{3}\right)$; ${ }^{1} \mathrm{H}$ NMR $\left(400 \mathrm{MHz}, \mathrm{CDCl}_{3}\right) \delta=9.86(\mathrm{~s}, 1 \mathrm{H}), 7.98(\mathrm{~d}, J=8.0 \mathrm{~Hz}, 2 \mathrm{H}), 7.58(\mathrm{t}, J=7.1 \mathrm{~Hz}, 1 \mathrm{H})$, $7.46(\mathrm{t}, J=7.6 \mathrm{~Hz}, 2 \mathrm{H}), 4.94(\mathrm{~d}, J=9.8 \mathrm{~Hz}, 2 \mathrm{H}), 4.87(\mathrm{td}, J=11.1,6.0 \mathrm{~Hz}, 1 \mathrm{H}), 4.51(\mathrm{~d}, J=$ $11.2 \mathrm{~Hz}, 1 \mathrm{H}), 4.36(\mathrm{~d}, J=11.3 \mathrm{~Hz}, 1 \mathrm{H}), 3.92(\mathrm{dd}, J=8.3,4.0 \mathrm{~Hz}, 1 \mathrm{H}), 2.50-2.33(\mathrm{~m}, 3 \mathrm{H})$, 2.33-2.19 (m, $6 \mathrm{H}), 2.14-2.02(\mathrm{~m}, 1 \mathrm{H}), 2.08(\mathrm{~s}, 3 \mathrm{H}), 1.95(\mathrm{~d}, J=8.4 \mathrm{~Hz}, 1 \mathrm{H}), 1.79$ (dt, $J=14.0$, $3.7 \mathrm{~Hz}, 1 \mathrm{H}), 1.74-1.62(\mathrm{~m}, 3 \mathrm{H}), 1.60-1.50(\mathrm{~m}, 1 \mathrm{H}), 1.44 \mathrm{ppm}(\mathrm{dd}, J=12.3,11.0 \mathrm{~Hz}, 1 \mathrm{H}) ;{ }^{13} \mathrm{C}$ NMR (100 MHz, $\left.\mathrm{CDCl}_{3}\right) \delta=224.1,203.1,169.7,166.2,142.9,133.3,130.0,129.6$ (2C), 128.7 (2C), 111.1, 69.7, 69.2, 68.0, 61.9, 52.5, 51.8, 51.7, 51.5, 42.8, 41.7, 38.3, 34.2, 30.5, 28.9 (2C), 21.6, 18.1 ppm; IR (film) $v_{\max }=2937,2851,2726,1719,1451,1387,1273,1112,1026,713 \mathrm{~cm}^{-1}$; HRMS (ESI): calcd for $\mathrm{C}_{29} \mathrm{H}_{32} \mathrm{NaO}_{7}^{+}[\mathrm{M}+\mathrm{Na}]^{+}$515.2040, found 515.2039.

\section{Preparation of aldoxime S-6:}

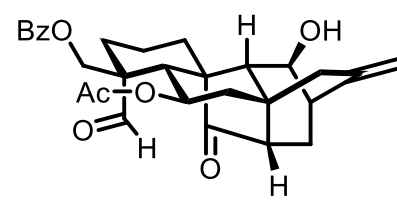

12

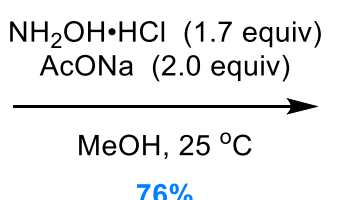

$76 \%$

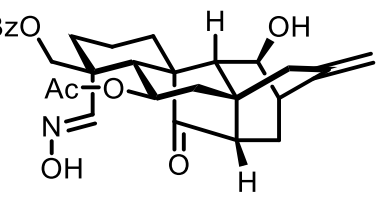

S-6 
To a stirred solution of keto-aldehyde 12 (15 mg, $30 \mu \mathrm{mol}, 1.0$ equiv) in $\mathrm{MeOH}(2 \mathrm{~mL})$ at 25 ${ }^{\circ} \mathrm{C}$ were sequentially added $\mathrm{NH}_{2} \mathrm{OH} \cdot \mathrm{HCl}(3.5 \mathrm{mg}, 50 \mu \mathrm{mol}, 1.7$ equiv) and $\mathrm{AcONa}(4.9 \mathrm{mg}, 60$ $\mu$ mol, 2.0 equiv). The resulting mixture was stirred for $1 \mathrm{~h}$ before it was added $\mathrm{NaHCO}_{3}(2 \mathrm{~mL}$, sat. aq.). The layers were separated, and the aqueous layer was extracted with EtOAc $(3 \times 2 \mathrm{~mL})$. The combined organic layers were dried $\left(\mathrm{Na}_{2} \mathrm{SO}_{4}\right)$ and concentrated in vacuo. Flash column chromatography (silica gel, $\mathrm{CH}_{2} \mathrm{Cl}_{2}$ :EtOAc 3:1) afforded aldoxime $\mathbf{S - 6}(12 \mathrm{mg}, 76 \%$ ) as a white amorphous solid. S-6: $R_{\mathrm{f}}=0.45$ (silica gel, $\mathrm{CH}_{2} \mathrm{Cl}_{2}:$ EtOAc $\left.3: 1\right) ;[\alpha]_{\mathrm{D}}{ }^{25}=+41.5\left(\mathrm{c}=0.40, \mathrm{CHCl}_{3}\right)$; ${ }^{1} \mathrm{H}$ NMR $\left(400 \mathrm{MHz}, \mathrm{CDCl}_{3}\right) \delta=8.07-8.00(\mathrm{~m}, 2 \mathrm{H}), 7.71(\mathrm{~s}, 1 \mathrm{H}), 7.62-7.56(\mathrm{~m}, 1 \mathrm{H}), 7.48(\mathrm{t}, J$ $=7.6 \mathrm{~Hz}, 2 \mathrm{H}), 7.22($ br s, $1 \mathrm{H}), 4.96-4.88(\mathrm{~m}, 2 \mathrm{H}), 4.76(\mathrm{td}, J=11.2,5.9 \mathrm{~Hz}, 1 \mathrm{H}), 4.66(\mathrm{~d}, J=$ $11.3 \mathrm{~Hz}, 1 \mathrm{H}), 4.30(\mathrm{~d}, J=11.3 \mathrm{~Hz}, 1 \mathrm{H}), 3.91(\mathrm{dd}, J=8.5,4.0 \mathrm{~Hz}, 1 \mathrm{H}), 2.42(\mathrm{dt}, J=18.2,2.8 \mathrm{~Hz}$, $1 \mathrm{H}), 2.37-2.25(\mathrm{~m}, 5 \mathrm{H}), 2.18(\mathrm{dd}, J=12.9,6.0 \mathrm{~Hz}, 1 \mathrm{H}), 2.12-2.02(\mathrm{~m}, 1 \mathrm{H}), 2.03(\mathrm{~s}, 3 \mathrm{H})$, 1.96-1.88 (m, $2 \mathrm{H}), 1.84$ (dd, $J=13.8,3.7 \mathrm{~Hz}, 1 \mathrm{H}), 1.80-1.62$ (m, $4 \mathrm{H}), 1.54-1.42$ ppm (m, $2 \mathrm{H})$; ${ }^{13} \mathrm{C}$ NMR $\left(100 \mathrm{MHz}, \mathrm{CDCl}_{3}\right) \delta=224.4,170.1,166.3,151.3,143.2,133.2,130.4,129.6(2 \mathrm{C})$, 128.7 (2C), 110.9, 70.7, 70.2, 68.1, 62.2, 52.6, 51.6, 51.5, 43.3, 42.8, 41.6, 38.3, 34.3, 33.8, 29.2 (2C), 21.7, 18.0 ppm; IR (film) $v_{\max }=3557,2941,2835,1722,1673,1583,1451,1382,1255$, 1100, 1038, 852, $726 \mathrm{~cm}^{-1}$; HRMS (ESI): calcd for $\mathrm{C}_{29} \mathrm{H}_{33} \mathrm{NNaO}_{7}^{+}\left[\mathrm{M}+\mathrm{Na}^{+} 530.2149\right.$, found 530.2156.

\section{Preparation of hexacycle 29:}

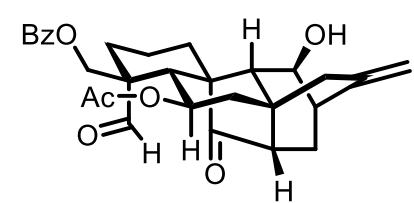

12

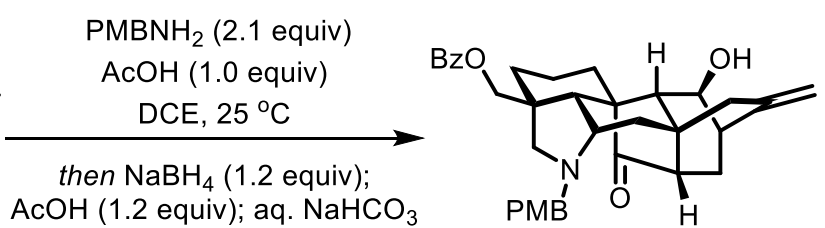

29

To a stirred solution of keto-aldehyde $12(0.40 \mathrm{~g}, 0.82 \mathrm{mmol}, 1.0$ equiv) in DCE (30 mL) at $25{ }^{\circ} \mathrm{C}$ were sequentially added $\mathrm{PMBNH}_{2}(0.22 \mathrm{~mL}, 1.7 \mathrm{mmol}, 2.1$ equiv) and $\mathrm{AcOH}(47 \mu \mathrm{L}, 0.82$ 
mmol, 1.0 equiv). The resulting mixture was stirred for $5 \mathrm{~h}$ before it was added $\mathrm{NaHCO}_{3}(15 \mathrm{~mL}$, sat. aq.). The layers were separated, and the aqueous layer was extracted with $\mathrm{CH}_{2} \mathrm{Cl}_{2}(3 \times 10 \mathrm{~mL})$. The combined organic layers were dried $\left(\mathrm{Na}_{2} \mathrm{SO}_{4}\right)$ and concentrated in vacuo. The residue was immediately dissolved in $\mathrm{MeOH}(30 \mathrm{~mL})$ at $0{ }^{\circ} \mathrm{C}$ was added $\mathrm{NaBH}_{4}(36 \mathrm{mg}, 0.95 \mathrm{mmol}, 1.2$ equiv). The resulting mixture was stirred for $0.5 \mathrm{~h}$ before it was sequentially added AcOH ( $54 \mu \mathrm{L}$, 0.95 mmol, 1.2 equiv) and $\mathrm{NaHCO}_{3}\left(30 \mathrm{~mL}\right.$, sat. aq.). The reaction was warmed to $25^{\circ} \mathrm{C}$ and stirred for $1 \mathrm{~h}$. The layers were separated, and the aqueous layer was extracted with EtOAc $(3 \times$ $15 \mathrm{~mL})$. The combined organic layers were washed with brine $(15 \mathrm{~mL})$, dried $\left(\mathrm{Na}_{2} \mathrm{SO}_{4}\right)$ and concentrated in vacuo. Flash column chromatography (silica gel, hexanes:EtOAc 1:1) afforded hexacycle $29(0.39 \mathrm{~g}, 85 \%)$ as a white amorphous solid (Note: after three parallel batches, $>1.1 \mathrm{~g}$ of 29 was obtained). 29: $R_{\mathrm{f}}=0.30$ (silica gel, hexanes:EtOAc $\left.1: 1\right) ;[\alpha]_{\mathrm{D}}^{25}=-21.6(\mathrm{c}=0.50$, $\left.\mathrm{CHCl}_{3}\right) ;{ }^{1} \mathrm{H} \mathrm{NMR}\left(400 \mathrm{MHz}, \mathrm{CDCl}_{3}\right) \delta=8.01-7.95(\mathrm{~m}, 2 \mathrm{H}), 7.59-7.54(\mathrm{~m}, 1 \mathrm{H}), 7.47-7.41(\mathrm{~m}$, $2 \mathrm{H}), 7.10(\mathrm{~d}, J=8.6 \mathrm{~Hz}, 2 \mathrm{H}), 6.86(\mathrm{~d}, J=8.6 \mathrm{~Hz}, 2 \mathrm{H}), 4.97-4.85(\mathrm{~m}, 2 \mathrm{H}), 4.14(\mathrm{~d}, J=10.9 \mathrm{~Hz}$, $1 \mathrm{H}), 4.12(\mathrm{~d}, J=13.8 \mathrm{~Hz}, 1 \mathrm{H}), 3.92(\mathrm{~d}, J=10.9 \mathrm{~Hz}, 1 \mathrm{H}), 3.78(\mathrm{~s}, 3 \mathrm{H}), 3.71(\mathrm{dd}, J=9.4,4.3 \mathrm{~Hz}$, $1 \mathrm{H}), 3.15(\mathrm{~d}, J=13.8 \mathrm{~Hz}, 1 \mathrm{H}), 3.04(\mathrm{t}, J=4.3 \mathrm{~Hz}, 1 \mathrm{H}), 2.75(\mathrm{~d}, J=10.4 \mathrm{~Hz}, 1 \mathrm{H}), 2.54(\mathrm{dd}, J=$ 10.3, 3.7 Hz, $1 \mathrm{H}), 2.47(\mathrm{dt}, J=18.1,2.7 \mathrm{~Hz}, 1 \mathrm{H}), 2.31-2.28(\mathrm{~m}, 1 \mathrm{H}), 2.27-2.21(\mathrm{~m}, 1 \mathrm{H}), 2.14$ $(\mathrm{d}, J=3.8 \mathrm{~Hz}, 1 \mathrm{H}), 2.08-1.96(\mathrm{~m}, 5 \mathrm{H}), 1.96-1.90(\mathrm{~m}, 1 \mathrm{H}), 1.88(\mathrm{~d}, J=9.0 \mathrm{~Hz}, 1 \mathrm{H}), 1.80-1.68$ (m, $3 \mathrm{H}), 1.59-1.48 \mathrm{ppm}(\mathrm{m}, 3 \mathrm{H}) ;{ }^{13} \mathrm{C} \mathrm{NMR}\left(100 \mathrm{MHz}, \mathrm{CDCl}_{3}\right) \delta=221.0,167.0,158.5,144.3$, 133.2, 130.7, 130.1, 129.7 (2C), 129.0 (2C), 128.6 (2C), 114.0 (2C), 110.1, 70.3, 68.7, 61.5, 60.9, $60.1,56.8,55.4,54.7,50.8,50.7,42.9$ (2C), 37.9, 36.7, 35.4, 30.0, 29.0, 26.9, 18.2 ppm; IR (film) $v_{\max }=2934,1716,1511,1271,1175,1109,1026,712 \mathrm{~cm}^{-1} ;$ HRMS (ESI): calcd for $\mathrm{C}_{35} \mathrm{H}_{40} \mathrm{NO}_{5}^{+}$ $[\mathrm{M}+\mathrm{H}]^{+}$554.2901, found 554.2906. 


\section{Preparation of hemiaminal 30:}

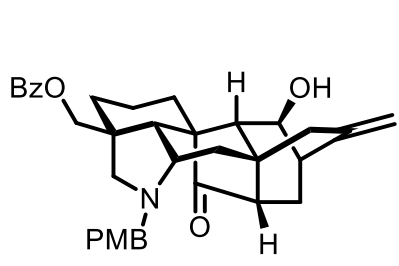

29
TBSOTf (1.1 equiv), $\mathrm{Et}_{3} \mathrm{~N}$ (1.5 equiv)

$\mathrm{CH}_{2} \mathrm{Cl}_{2}, 25^{\circ} \mathrm{C}$; DDQ (2.0 equiv)

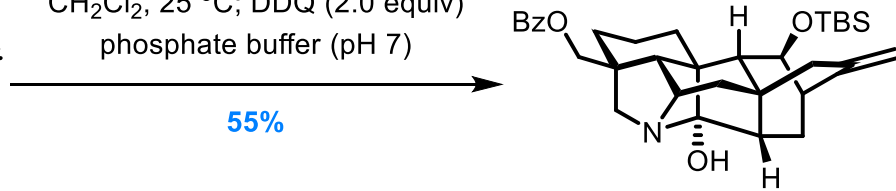

30

To a stirred solution of hexacycle $29\left(0.17 \mathrm{~g}, 0.30 \mathrm{mmol}, 1.0\right.$ equiv) in $\mathrm{CH}_{2} \mathrm{Cl}_{2}(10 \mathrm{~mL})$ at 25 ${ }^{\circ} \mathrm{C}$ were sequentially added $\mathrm{Et}_{3} \mathrm{~N}(63 \mu \mathrm{L}, 0.45 \mathrm{mmol}, 1.5$ equiv) and TBSOTf (76 $\mu \mathrm{L}, 0.33 \mathrm{mmol}$, 1.1 equiv). The resulting mixture was stirred for $0.5 \mathrm{~h}$ before it was added $\mathrm{pH} 7$ phosphate buffer (10 mL, $2.0 \mathrm{M}$ aq.) and DDQ (0.14 g, $0.60 \mathrm{mmol}, 2.0$ equiv). The resulting mixture was stirred for an additional $10 \mathrm{~h}$ before it was added $\mathrm{NaHCO}_{3}(10 \mathrm{~mL}$, sat. aq.). The layers were separated, and the aqueous layer was extracted with $\mathrm{CH}_{2} \mathrm{Cl}_{2}(3 \times 10 \mathrm{~mL})$. The combined organic layers were dried $\left(\mathrm{Na}_{2} \mathrm{SO}_{4}\right)$ and concentrated in vacuo. Flash column chromatography (silica gel, $\left.\mathrm{CH}_{2} \mathrm{Cl}_{2}: \mathrm{MeOH} 10: 1\right)$ afforded hemiaminal $30(90 \mathrm{mg}, 55 \%)$ as a colorless oil. 30: $R_{\mathrm{f}}=0.45$ (silica gel, $\left.\mathrm{CH}_{2} \mathrm{Cl}_{2}: \mathrm{MeOH} 10: 1\right) ;[\alpha]_{\mathrm{D}}{ }^{25}=+9.4\left(\mathrm{c}=0.40, \mathrm{CHCl}_{3}\right) ;{ }^{1} \mathrm{H} \mathrm{NMR}\left(400 \mathrm{MHz}, \mathrm{CDCl}_{3}\right) \delta=8.02$ $(\mathrm{d}, J=7.4 \mathrm{~Hz}, 2 \mathrm{H}), 7.61(\mathrm{t}, J=7.4 \mathrm{~Hz}, 1 \mathrm{H}), 7.48(\mathrm{t}, J=7.6 \mathrm{~Hz}, 2 \mathrm{H}), 4.72(\mathrm{~d}, J=5.9 \mathrm{~Hz}, 2 \mathrm{H})$, $4.35(\mathrm{~d}, J=11.3 \mathrm{~Hz}, 1 \mathrm{H}), 4.17(\mathrm{~s}, 1 \mathrm{H}), 4.15(\mathrm{~d}, J=11.3 \mathrm{~Hz}, 1 \mathrm{H}), 4.02(\mathrm{~d}, J=4.6 \mathrm{~Hz}, 1 \mathrm{H}), 3.95$ $(\mathrm{d}, J=12.7 \mathrm{~Hz}, 1 \mathrm{H}), 3.20(\mathrm{~d}, J=12.7 \mathrm{~Hz}, 1 \mathrm{H}), 2.83(\mathrm{~d}, J=10.4 \mathrm{~Hz}, 1 \mathrm{H}), 2.36-2.16(\mathrm{~m}, 5 \mathrm{H})$, 2.08-2.00 (m, $2 \mathrm{H}), 1.96-1.77(\mathrm{~m}, 5 \mathrm{H}), 1.72-1.66(\mathrm{~m}, 1 \mathrm{H}), 1.60-1.54(\mathrm{~m}, 1 \mathrm{H}), 1.44(\mathrm{~d}, J=2.3$ $\mathrm{Hz}, 1 \mathrm{H}), 1.33-1.26(\mathrm{~m}, 1 \mathrm{H}), 0.84(\mathrm{~s}, 9 \mathrm{H}), 0.03 \mathrm{ppm}(\mathrm{s}, 6 \mathrm{H}) ;{ }^{13} \mathrm{C} \mathrm{NMR}\left(100 \mathrm{MHz}, \mathrm{CDCl}_{3}\right) \delta=$ 166.4, 144.5, 133.7, 129.8 (2C), 129.4, 128.8 (2C), 108.6, 104.1, 70.1, 68.2, 64.3, 62.4, 54.7, 49.0, 48.6, 48.3, 41.4, 41.0, 40.8, 33.9, 33.2, 28.4, 25.9 (3C), 24.6, 24.2, 18.0, 17.4, -4.1, -4.4 ppm; IR (film) $v_{\max }=2927,2830,1720,1271,1183,1089,712 \mathrm{~cm}^{-1} ;$ HRMS (ESI): calcd for $\mathrm{C}_{33} \mathrm{H}_{46} \mathrm{NO}_{4} \mathrm{Si}^{+}[\mathrm{M}+\mathrm{H}]^{+}$548.3191, found 548.3196. 


\section{Preparation of thiocarbamate 31:}

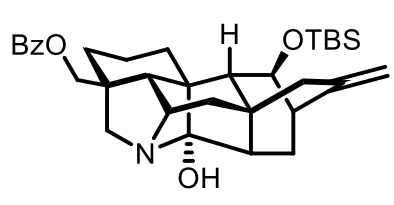

30

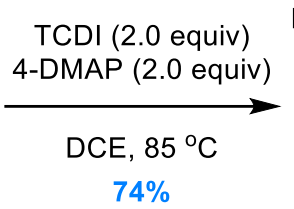

$74 \%$

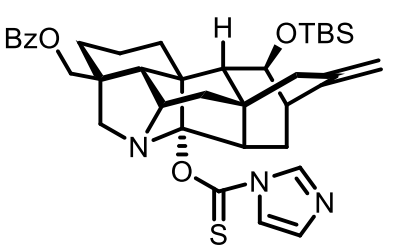

31

To a stirred solution of hemiaminal 30 (0.33 g, $0.60 \mathrm{mmol}, 1.0$ equiv) in DCE (50 mL) at 25 ${ }^{\circ} \mathrm{C}$ were sequentially added TCDI (0.21 g, $1.2 \mathrm{mmol}, 2.0$ equiv) and 4-DMAP (0.15 g, $1.2 \mathrm{mmol}$, 2.0 equiv). The resulting mixture was warmed to $85^{\circ} \mathrm{C}$ and stirred for $9 \mathrm{~h}$ before it was cooled to $25^{\circ} \mathrm{C}$ and added $\mathrm{NaHCO}_{3}$ (30 mL, sat. aq.). The layers were separated, and the aqueous layer was extracted with $\mathrm{CH}_{2} \mathrm{Cl}_{2}(3 \times 20 \mathrm{~mL})$. The combined organic layers were dried $\left(\mathrm{Na}_{2} \mathrm{SO}_{4}\right)$ and concentrated in vacuo. Flash column chromatography (silica gel, hexanes:EtOAc 1:1) afforded thiocarbamate $31(0.29 \mathrm{~g}, 74 \%)$ as a yellow amorphous solid. 31: $R_{\mathrm{f}}=0.35$ (silica gel, hexanes:EtOAc 1:1); $[\alpha]_{\mathrm{D}}{ }^{25}=+65.7\left(\mathrm{c}=0.40, \mathrm{CHCl}_{3}\right) ;{ }^{1} \mathrm{H} \mathrm{NMR}\left(400 \mathrm{MHz}, \mathrm{CDCl}_{3}\right) \delta=8.33(\mathrm{~s}, 1$ H), 8.07-7.97 (m, 2 H), 7.67 (s, $1 \mathrm{H}), 7.61-7.53$ (m, 1 H), 7.51-7.40 (m, $2 \mathrm{H}), 7.08$ (s, $1 \mathrm{H}), 4.69$ $(\mathrm{s}, 2 \mathrm{H}), 4.30(\mathrm{~d}, J=11.1 \mathrm{~Hz}, 1 \mathrm{H}), 4.10(\mathrm{~d}, J=11.1 \mathrm{~Hz}, 1 \mathrm{H}), 4.06(\mathrm{~d}, J=4.7 \mathrm{~Hz}, 1 \mathrm{H}), 3.55(\mathrm{~s}, 1$ H), $3.09(\mathrm{dt}, J=10.7,2.6 \mathrm{~Hz}, 1 \mathrm{H}), 2.83(\mathrm{~d}, J=13.7 \mathrm{~Hz}, 1 \mathrm{H}), 2.69(\mathrm{~d}, J=13.7 \mathrm{~Hz}, 1 \mathrm{H}), 2.32(\mathrm{dt}$, $J=17.5,2.7 \mathrm{~Hz}, 1 \mathrm{H}), 2.25-2.21(\mathrm{~m}, 1 \mathrm{H}), 2.18(\mathrm{dt}, J=17.5,2.7 \mathrm{~Hz}, 1 \mathrm{H}), 2.06(\mathrm{~d}, J=13.4 \mathrm{~Hz}, 1$ H), 1.90-1.79 (m, 4 H), 1.76-1.65 (m, 4 H), 1.57 (ddd, $J=18.8,11.7,4.1 \mathrm{~Hz}, 1 \mathrm{H}), 1.45$ (d, $J=$ $1.8 \mathrm{~Hz}, 1 \mathrm{H}), 1.28(\mathrm{dt}, J=14.8,2.9 \mathrm{~Hz}, 1 \mathrm{H}), 0.85(\mathrm{~s}, 9 \mathrm{H}), 0.07(\mathrm{~s}, 3 \mathrm{H}), 0.04 \mathrm{ppm}(\mathrm{s}, 3 \mathrm{H}) ;{ }^{13} \mathrm{C}$ NMR (100 MHz, $\left.\mathrm{CDCl}_{3}\right) \delta=179.9,166.8,145.7,136.3,133.3,131.2,130.0,129.7$ (2C), 128.6 (2C), 118.4, 112.9, 108.1, 71.2, 68.5, 64.3, 61.5, 57.9, 51.9, 50.9, 46.3, 41.8 (2C), 41.2, 35.4, 34.1, 28.0, 25.9 (3C), 25.0, 24.3, 18.4, 18.0, -4.1, -4.3 ppm; IR (film) $v_{\max }=2923,2836,1721,1703$, 1655, 1322, 1052, $724 \mathrm{~cm}^{-1}$; HRMS (ESI): calcd for $\mathrm{C}_{37} \mathrm{H}_{48} \mathrm{~N}_{3} \mathrm{O}_{4} \mathrm{SSi}^{+}[\mathrm{M}+\mathrm{H}]^{+} 658.3129$, found 658.3137. 


\section{Preparation of (+)-18-benzoyldavisinol (8):}

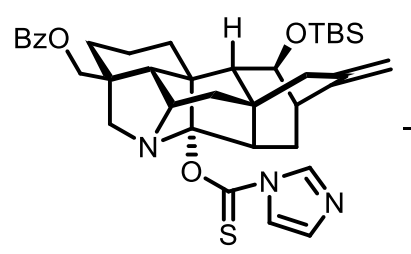

31

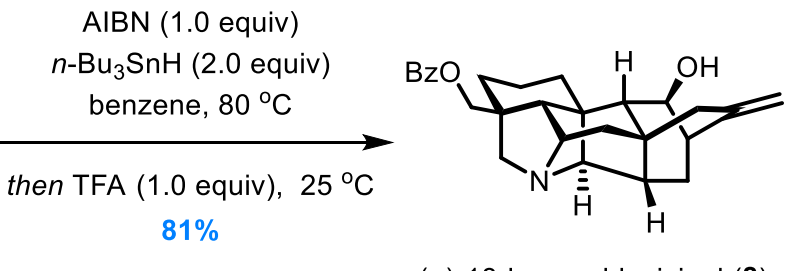

(+)-18-benzoyldavisinol (8)

To a stirred solution of thiocarbamate 31 ( $0.16 \mathrm{~g}, 0.24 \mathrm{mmol}, 1.0$ equiv) in benzene $(10 \mathrm{~mL})$ at $25{ }^{\circ} \mathrm{C}$ were sequentially added $\mathrm{AIBN}$ (40 mg, $0.24 \mathrm{mmol}, 1.0$ equiv) and $n$ - $\mathrm{Bu}_{3} \mathrm{SnH}(0.13 \mathrm{~mL}$, $0.48 \mathrm{mmol}, 2.0$ equiv). The resulting mixture was warmed to $80{ }^{\circ} \mathrm{C}$ and stirred for $0.5 \mathrm{~h}$ before it was cooled to $25{ }^{\circ} \mathrm{C}$ and added TFA ( $18 \mu \mathrm{L}, 0.24 \mathrm{mmol}, 1.0$ equiv). The reaction was stirred for an additional $12 \mathrm{~h}$ before it was added $\mathrm{NaHCO}_{3}(10 \mathrm{~mL}$, sat. aq.). The layers were separated, and the aqueous layer was extracted with EtOAc $(5 \times 10 \mathrm{~mL})$. The combined organic layers were dried $\left(\mathrm{Na}_{2} \mathrm{SO}_{4}\right)$ and concentrated in vacuo. Flash column chromatography (silica gel, $\mathrm{CH}_{2} \mathrm{Cl}_{2}: \mathrm{MeOH}$ 10:1) afforded (+)-18-benzoyldavisinol (8, $\left.84 \mathrm{mg}, 81 \%\right)$ as a white amorphous solid. (+)-8: $R_{\mathrm{f}}=0.35$ (silica gel, $\left.\mathrm{CH}_{2} \mathrm{Cl}_{2}: \mathrm{MeOH} 10: 1\right) ;[\alpha]_{\mathrm{D}}^{25}=+43.5\left(\mathrm{c}=0.33, \mathrm{CHCl}_{3}\right)$ [Lit. $\left.[\alpha]_{\mathrm{D}}{ }^{25}=+42.34\left(\mathrm{c}=0.206, \mathrm{CHCl}_{3}\right)\right] ;{ }^{1} \mathrm{H} \mathrm{NMR}\left(400 \mathrm{MHz}, \mathrm{CDCl}_{3}\right) \delta=8.02(\mathrm{~d}, J=7.2 \mathrm{~Hz}, 2 \mathrm{H})$, $7.58(\mathrm{t}, J=7.4 \mathrm{~Hz}, 1 \mathrm{H}), 7.46(\mathrm{t}, J=7.7 \mathrm{~Hz}, 2 \mathrm{H}), 4.89($ br s, $2 \mathrm{H}), 4.23(\mathrm{~d}, J=11.0 \mathrm{~Hz}, 1 \mathrm{H}), 4.07$ $(\mathrm{d}, J=4.8 \mathrm{~Hz}, 1 \mathrm{H}), 4.05(\mathrm{~d}, J=11.0 \mathrm{~Hz}, 1 \mathrm{H}), 3.24(\mathrm{br} \mathrm{s}, 1 \mathrm{H}), 2.70(\mathrm{~d}, J=12.9 \mathrm{~Hz}, 1 \mathrm{H}), 2.48(\mathrm{~s}$, $1 \mathrm{H}), 2.43(\mathrm{~d}, J=12.8 \mathrm{~Hz}, 1 \mathrm{H}), 2.36-2.31(\mathrm{~m}, 1 \mathrm{H}), 2.27(\mathrm{dt}, J=17.8,2.6 \mathrm{~Hz}, 1 \mathrm{H}), 2.20(\mathrm{dt}, J=$ 17.9, $2.3 \mathrm{~Hz}, 1 \mathrm{H}), 2.00-1.84(\mathrm{~m}, 4 \mathrm{H}), 1.78-1.71(\mathrm{~m}, 2 \mathrm{H}), 1.63-1.49$ (m, $5 \mathrm{H}), 1.44$ (s, $1 \mathrm{H})$, $1.02 \mathrm{ppm}(\mathrm{dt}, J=13.8,2.8 \mathrm{~Hz}, 1 \mathrm{H}) ;{ }^{13} \mathrm{C} \mathrm{NMR}\left(100 \mathrm{MHz}, \mathrm{CDCl}_{3}\right) \delta=166.6,145.6,133.1,130.1$, $129.5(2 \mathrm{C}), 128.4(2 \mathrm{C}), 110.5,75.9,70.8,67.5,65.1,59.6,58.4,56.4,49.6,44.4,42.3,41.9,40.5$, 35.8, 33.6, 29.5, 28.9, 26.5, 18.8 ppm; IR (film) $v_{\max }=1726,1469,1372,1338,1277 \mathrm{~cm}^{-1}[$ Lit. found IR (nujol) $\left.v_{\max }=1720,1465,1375,1336,1270 \mathrm{~cm}^{-1}\right]{ }^{5}$ HRMS (ESI): calcd for $\mathrm{C}_{27} \mathrm{H}_{32} \mathrm{NO}_{3}{ }^{+}$ $[\mathrm{M}+\mathrm{H}]^{+}$418.2377, found 418.2382 [Lit. found 418.2384]. ${ }^{5}$ 


\section{Preparation of $(+)$-davisinol (7):}

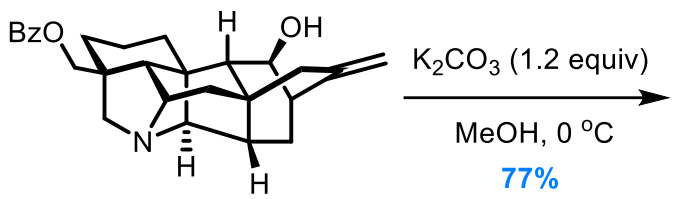

(+)-18-benzoyldavisinol (8)

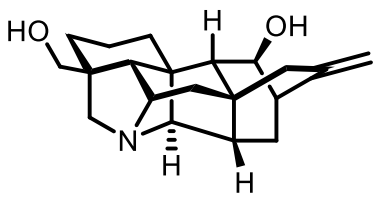

(+)-davisinol (7)

To a stirred solution of (+)-18-benzoyldavisinol (8, $84 \mathrm{mg}, 0.20 \mathrm{mmol}, 1.0$ equiv) in $\mathrm{MeOH}$ $(10 \mathrm{~mL})$ at $0{ }^{\circ} \mathrm{C}$ was added $\mathrm{K}_{2} \mathrm{CO}_{3}(34 \mathrm{mg}, 0.24 \mathrm{mmol}, 1.2$ equiv). The resulting mixture was stirred for $5 \mathrm{~h}$ before it was concentrated in vacuo. Flash column chromatography (basic aluminium oxide, $\mathrm{CH}_{2} \mathrm{Cl}_{2}: \mathrm{MeOH}$ 5:1) afforded (+)-davisinol (7, $49 \mathrm{mg}, 77 \%$ ) as a white amorphous solid. (+)-7: $R_{\mathrm{f}}=0.40$ (silica gel, $\left.\mathrm{CH}_{2} \mathrm{Cl}_{2}: \mathrm{MeOH} 5: 1\right) ;[\alpha]_{\mathrm{D}}^{25}=+31.5(\mathrm{c}=0.33$, $\left.\mathrm{CHCl}_{3}\right)\left[\right.$ Lit. $\left.[\alpha]_{\mathrm{D}}{ }^{25}=+27.5\left(\mathrm{c}=0.189, \mathrm{CHCl}_{3}\right)\right] ;{ }^{1} \mathrm{H}$ NMR $\left(400 \mathrm{MHz}, \mathrm{CDCl}_{3}\right) \delta=4.84(\mathrm{~d}, J=$ $2.2 \mathrm{~Hz}, 2 \mathrm{H}), 4.01(\mathrm{~d}, J=4.7 \mathrm{~Hz}, 1 \mathrm{H}), 3.45(\mathrm{~d}, J=10.7 \mathrm{~Hz}, 1 \mathrm{H}), 3.28(\mathrm{~d}, J=10.7 \mathrm{~Hz}, 1 \mathrm{H})$, $3.12($ br s, $1 \mathrm{H}), 2.55(\mathrm{~d}, J=12.8 \mathrm{~Hz}, 1 \mathrm{H}), 2.40(\mathrm{~s}, 1 \mathrm{H}), 2.30-2.26(\mathrm{~m}, 1 \mathrm{H}), 2.23(\mathrm{~d}, J=12.6$ $\mathrm{Hz}, 1 \mathrm{H}), 2.21(\mathrm{dt}, J=17.9,2.4 \mathrm{~Hz}, 1 \mathrm{H}), 2.14(\mathrm{dt}, J=17.9,2.4 \mathrm{~Hz}, 1 \mathrm{H}), 1.94-1.86(\mathrm{~m}, 2 \mathrm{H})$, 1.85-1.77 (m, $3 \mathrm{H}), 1.73-1.69(\mathrm{~m}, 1 \mathrm{H}), 1.72(\mathrm{~s}, 1 \mathrm{H}), 1.66(\mathrm{dd}, J=13.4,3.1 \mathrm{~Hz}, 1 \mathrm{H}), 1.52(\mathrm{dd}$, $J=13.6,2.3 \mathrm{~Hz}, 1 \mathrm{H}), 1.49-1.38(\mathrm{~m}, 4 \mathrm{H}), 1.36(\mathrm{~s}, 1 \mathrm{H}), 0.95 \mathrm{ppm}(\mathrm{dt}, J=13.8,2.9 \mathrm{~Hz}, 1 \mathrm{H})$; ${ }^{13} \mathrm{C}$ NMR $\left(100 \mathrm{MHz}, \mathrm{CDCl}_{3}\right) \delta=145.6,110.5,75.8,69.6,67.5,64.9,59.6,58.3,56.0,49.6,44.2$, 43.5, 41.9, 40.4, 35.8, 33.6, 29.5, 28.3, 26.5, 18.8 ppm; IR (film) $v_{\max }=3349,1108,750,721$ $\mathrm{cm}^{-1}\left[\right.$ Lit. found IR (nujol) $\left.v_{\max }=3345,1100,750,725 \mathrm{~cm}^{-1}\right],^{5}$ HRMS (ESI): calcd for $\mathrm{C}_{20} \mathrm{H}_{28} \mathrm{NO}_{2}{ }^{+}[\mathrm{M}+\mathrm{H}]^{+}$314.2115, found 314.2119 [Lit. found 314.2108]. ${ }^{5}$

\section{Conversion of (+)-davisinol (7) into (+)-18-benzoyldavisinol (8):}

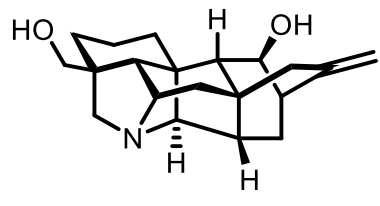

(+)-davisinol (7)

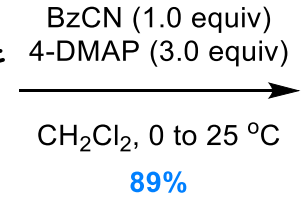

$89 \%$

(+)-18-benzoyldavisinol (8)

To a stirred solution of (+)-davisinol (7, $9.4 \mathrm{mg}, 30 \mu \mathrm{mol}, 1.0$ equiv) in $\mathrm{CH}_{2} \mathrm{Cl}_{2}(2 \mathrm{~mL})$ at 0 
${ }^{\circ} \mathrm{C}$ were sequentially added 4-DMAP (11 mg, $90 \mu \mathrm{mol}, 3.0$ equiv) and BzCN (3.9 mg, $30 \mu \mathrm{mol}$, 1.0 equiv). The resulting mixture was warmed to $25^{\circ} \mathrm{C}$ and stirred for $0.5 \mathrm{~h}$ before it was added $\mathrm{NaHCO}_{3}(2 \mathrm{~mL}$, sat. aq.). The layers were separated, and the aqueous layer was extracted with $\mathrm{CH}_{2} \mathrm{Cl}_{2}(3 \times 2 \mathrm{~mL})$. The combined organic layers were dried $\left(\mathrm{Na}_{2} \mathrm{SO}_{4}\right)$ and concentrated in vacuo Flash column chromatography (silica gel, $\mathrm{CH}_{2} \mathrm{Cl}_{2}: \mathrm{MeOH}$ 10:1) afforded (+)-18-benzoyldavisinol (8, $11 \mathrm{mg}, 89 \%)$. 
Table S3. ${ }^{1} \mathrm{H}$ NMR $\left(\mathrm{CDCl}_{3}, 25{ }^{\circ} \mathrm{C}\right)$ Spectroscopic Comparison of Natural ${ }^{5}$ and Synthetic (+)-Davisinol (7).

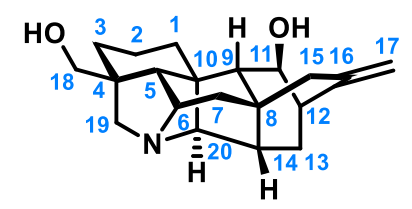

(+)-davisinol (7)

\begin{tabular}{|c|c|c|}
\hline No. & $\begin{array}{c}\text { Natural } \\
\delta{ }^{1} \mathrm{H}[\mathrm{ppm}, \text { mult, } J(\mathrm{~Hz})]\end{array}$ & $\begin{array}{c}\text { Synthetic } \\
\delta{ }^{1} \mathrm{H}[\mathrm{ppm}, \text { mult, } J(\mathrm{~Hz})]\end{array}$ \\
\hline $1 \mathrm{a}$ & $1.80(\mathrm{~m})$ & $1.85-1.77(\mathrm{~m})$ \\
\hline $1 b$ & $1.40(\mathrm{~m})$ & $1.49-1.38(\mathrm{~m})$ \\
\hline $2 \mathrm{a}$ & $1.70(\mathrm{~m})$ & $1.73-1.69(\mathrm{~m})$ \\
\hline $2 b$ & $1.50-1.40(\mathrm{~m})$ & $1.49-1.38(\mathrm{~m})$ \\
\hline 3 & $1.48(\mathrm{~m})$ & $1.49-1.38(\mathrm{~m})$ \\
\hline \multicolumn{3}{|l|}{4} \\
\hline 5 & $1.72(\mathrm{~s})$ & $1.72(\mathrm{~s})$ \\
\hline 6 & 3.14 (br s) & 3.12 (br s) \\
\hline $7 \mathrm{a}$ & $1.65(\mathrm{~m})$ & $1.66(\mathrm{dd}, J=13.4,3.1 \mathrm{~Hz})$ \\
\hline $7 b$ & $1.57(\mathrm{~m})$ & $1.52(\mathrm{dd}, J=13.6,2.3 \mathrm{~Hz})$ \\
\hline \multicolumn{3}{|l|}{8} \\
\hline 9 & $1.38(\mathrm{~s})$ & $1.36(\mathrm{~s})$ \\
\hline \multicolumn{3}{|l|}{10} \\
\hline 11 & $4.01(\mathrm{~d}, J=4.8 \mathrm{~Hz})$ & $4.01(\mathrm{~d}, J=4.7 \mathrm{~Hz})$ \\
\hline 12 & $2.28(\mathrm{~d}, J=4.8 \mathrm{~Hz})$ & $2.30-2.26(\mathrm{~m})$ \\
\hline $13 \mathrm{a}$ & $1.90(\mathrm{~m})$ & $1.94-1.86(\mathrm{~m})$ \\
\hline $13 b$ & $0.91(\mathrm{~m})$ & $0.95(\mathrm{dt}, J=13.8,2.9 \mathrm{~Hz})$ \\
\hline 14 & $1.78(\mathrm{~m})$ & $1.85-1.77(\mathrm{~m})$ \\
\hline $15 \mathrm{a}$ & $2.10(\mathrm{~m})$ & $2.21(\mathrm{dt}, J=17.9,2.4 \mathrm{~Hz})$ \\
\hline $15 b$ & & $2.14(\mathrm{dt}, J=17.9,2.4 \mathrm{~Hz})$ \\
\hline \multicolumn{3}{|r|}{ - } \\
\hline 17 & $4.83(\mathrm{~d}, J=1.8 \mathrm{~Hz})$ & $4.84(\mathrm{~d}, J=2.2 \mathrm{~Hz})$ \\
\hline $18 \mathrm{a}$ & $3.43(\mathrm{~d}, J=10.8 \mathrm{~Hz})$ & $3.45(\mathrm{~d}, J=10.7 \mathrm{~Hz})$ \\
\hline $18 \mathrm{~b}$ & $3.28(\mathrm{~d}, J=10.8 \mathrm{~Hz})$ & $3.28(\mathrm{~d}, J=10.7 \mathrm{~Hz})$ \\
\hline $19 \mathrm{a}$ & $2.55(\mathrm{~d}, J=12.5 \mathrm{~Hz})$ & $2.55(\mathrm{~d}, J=12.8 \mathrm{~Hz})$ \\
\hline $19 b$ & $2.23(\mathrm{~d}, J=12.5 \mathrm{~Hz})$ & $2.23(\mathrm{~d}, J=12.6 \mathrm{~Hz})$ \\
\hline 20 & $2.40(\mathrm{~s})$ & $2.40(\mathrm{~s})$ \\
\hline
\end{tabular}


Table S4. ${ }^{13} \mathrm{C}$ NMR $\left(\mathrm{CDCl}_{3}, 25{ }^{\circ} \mathrm{C}\right)$ Spectroscopic Comparison of Natural ${ }^{5}$ and Synthetic (+)-Davisinol (7).

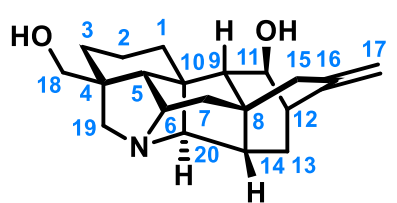

(+)-davisinol (7)

\begin{tabular}{ccc}
\hline No. & $\begin{array}{c}\text { Natural } \\
\delta^{13} \mathrm{C}(\mathrm{ppm})\end{array}$ & $\begin{array}{c}\text { Synthetic } \\
\delta^{13} \mathrm{C}(\mathrm{ppm})\end{array}$ \\
\hline 1 & 26.5 & 26.5 \\
2 & 18.9 & 18.8 \\
3 & 28.4 & 28.3 \\
4 & 43.5 & 43.5 \\
5 & 56.0 & 56.0 \\
6 & 64.8 & 64.9 \\
7 & 35.8 & 35.8 \\
8 & 40.5 & 40.4 \\
9 & 59.5 & 59.6 \\
10 & 49.6 & 49.6 \\
11 & 67.4 & 67.5 \\
12 & 41.9 & 41.9 \\
13 & 29.6 & 29.5 \\
14 & 44.0 & 44.2 \\
15 & 33.6 & 33.6 \\
16 & 145.8 & 145.6 \\
17 & 110.1 & 110.5 \\
18 & 69.2 & 69.6 \\
19 & 58.2 & 58.3 \\
20 & 75.7 & 75.8 \\
\hline
\end{tabular}


Table S5. ${ }^{1} \mathrm{H}$ NMR $\left(\mathrm{CDCl}_{3}, 25{ }^{\circ} \mathrm{C}\right)$ Spectroscopic Comparison of Natural ${ }^{5}$ and Synthetic (+)-18-Benzoyldavisinol (8).

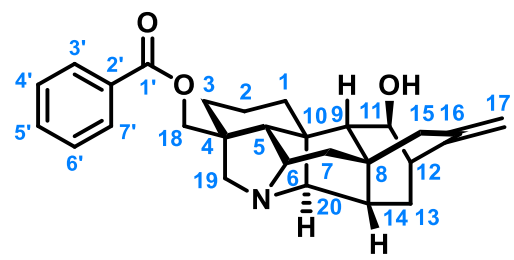

(+)-18-benzoyldavisinol (8)

\begin{tabular}{|c|c|c|}
\hline No. & $\begin{array}{c}\text { Natural } \\
\delta{ }^{1} \mathrm{H}[\mathrm{ppm}, \text { mult, } J(\mathrm{~Hz})]\end{array}$ & $\begin{array}{c}\text { Synthetic* } \\
\delta^{1} \mathrm{H}[\mathrm{ppm}, \text { mult, } J(\mathrm{~Hz})]\end{array}$ \\
\hline $1 \mathrm{a}$ & $1.92(\mathrm{~m})$ & $2.00-1.84(\mathrm{~m})$ \\
\hline $1 b$ & $1.51(\mathrm{~m})$ & $1.63-1.49(\mathrm{~m})$ \\
\hline $2 \mathrm{a}$ & $1.79(\mathrm{~m})$ & $1.78-1.71(\mathrm{~m})$ \\
\hline $2 b$ & $1.51(\mathrm{~m})$ & $1.63-1.49(\mathrm{~m})$ \\
\hline 3 & $1.62(\mathrm{~m})$ & $1.63-1.49(\mathrm{~m})$ \\
\hline \multicolumn{3}{|l|}{4} \\
\hline 5 & $1.88(\mathrm{~m})$ & $2.00-1.84(\mathrm{~m})$ \\
\hline 6 & 3.27 (br s) & 3.24 (br s) \\
\hline $7 \mathrm{a}$ & $1.76(\mathrm{~m})$ & $1.78-1.71(\mathrm{~m})$ \\
\hline $7 b$ & $1.61(\mathrm{~m})$ & $1.63-1.49(\mathrm{~m})$ \\
\hline \multicolumn{3}{|l|}{8} \\
\hline 9 & $1.45(\mathrm{~s})$ & $1.44(\mathrm{~s})$ \\
\hline \multicolumn{3}{|l|}{10} \\
\hline 11 & $4.07(\mathrm{~d}, J=4.8 \mathrm{~Hz})$ & $4.07(\mathrm{~d}, J=4.8 \mathrm{~Hz})$ \\
\hline 12 & 2.33 (br s $1 / 2$ w 9$)$ & $2.36-2.31(\mathrm{~m})$ \\
\hline $13 \mathrm{a}$ & $1.95(\mathrm{~m})$ & $2.00-1.84(\mathrm{~m})$ \\
\hline $13 b$ & $1.02(\mathrm{~m})$ & $1.02(\mathrm{dt}, J=13.8,2.8 \mathrm{~Hz})$ \\
\hline 14 & $1.90(\mathrm{~m})$ & $2.00-1.84(\mathrm{~m})$ \\
\hline $15 \mathrm{a}$ & $2.27(\mathrm{~m})$ & $2.27(\mathrm{dt}, J=17.8,2.6 \mathrm{~Hz})$ \\
\hline $15 b$ & $2.20(\mathrm{~m})$ & $2.20(\mathrm{dt}, J=17.9,2.3 \mathrm{~Hz})$ \\
\hline \multicolumn{3}{|r|}{ 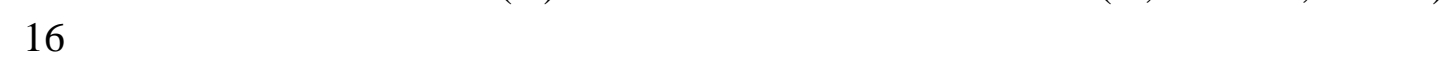 } \\
\hline 17 & 4.89 (br s) & 4.89 (br s) \\
\hline $18 \mathrm{a}$ & $4.24(\mathrm{~d}, J=12.8 \mathrm{~Hz})$ & $4.23(\mathrm{~d}, J=11.0 \mathrm{~Hz})$ \\
\hline $18 b$ & $4.06(\mathrm{~d}, J=12.8 \mathrm{~Hz})$ & $4.05(\mathrm{~d}, J=11.0 \mathrm{~Hz})$ \\
\hline $19 \mathrm{a}$ & $2.72(\mathrm{~d}, J=17.9 \mathrm{~Hz})$ & $2.70(\mathrm{~d}, J=12.9 \mathrm{~Hz})$ \\
\hline $19 b$ & $2.44(\mathrm{~d}, J=17.9 \mathrm{~Hz})$ & $2.43(\mathrm{~d}, J=12.8 \mathrm{~Hz})$ \\
\hline 20 & $2.51(\mathrm{~s})$ & $2.48(\mathrm{~s})$ \\
\hline \multicolumn{3}{|l|}{$1^{\prime}$} \\
\hline \multicolumn{3}{|l|}{$2^{\prime}$} \\
\hline $3^{\prime}, 7^{\prime}$ & $8.02(\mathrm{~d}, J=7.5 \mathrm{~Hz})$ & $8.02(\mathrm{~d}, J=7.2 \mathrm{~Hz})$ \\
\hline $4^{\prime}, 6^{\prime}$ & $7.46(\mathrm{dd}, J=7.6 \mathrm{~Hz})$ & $7.46(\mathrm{t}, J=7.7 \mathrm{~Hz})$ \\
\hline $5^{\prime}$ & $7.58(\mathrm{dd}, J=7.4 \mathrm{~Hz})$ & $7.58(\mathrm{t}, J=7.4 \mathrm{~Hz})$ \\
\hline
\end{tabular}

*The synthetic sample was bubbled with a stream of $\mathrm{NH}_{3(\mathrm{~g})}$ for ca. $3 \mathrm{sec}$ in $\mathrm{CDCl}_{3}$ prior to $\mathrm{NMR}$ experiments. 
Table S6. ${ }^{13} \mathrm{C}$ NMR $\left(\mathrm{CDCl}_{3}, 25{ }^{\circ} \mathrm{C}\right)$ Spectroscopic Comparison of Natural ${ }^{5}$ and Synthetic (+)-18-Benzoyldavisinol (8).

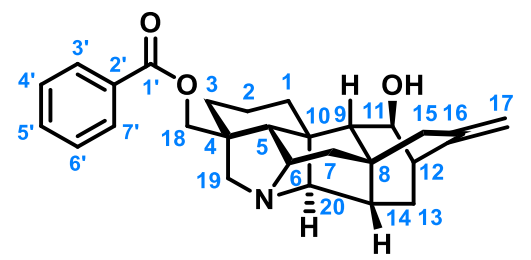

(+)-18-benzoyldavisinol (8)

\begin{tabular}{ccc}
\hline No. & $\begin{array}{c}\text { Natural } \\
{ }^{13} \mathrm{C}(\mathrm{ppm})\end{array}$ & $\begin{array}{c}\text { Synthetic* } \\
{ }^{13} \mathrm{C}(\mathrm{ppm})\end{array}$ \\
\hline 1 & 26.4 & 26.5 \\
2 & 18.8 & 18.8 \\
3 & 28.9 & 28.9 \\
4 & 42.3 & 42.3 \\
5 & 56.3 & 56.4 \\
6 & 65.2 & 65.1 \\
7 & 35.8 & 35.8 \\
8 & 40.5 & 40.5 \\
9 & 59.6 & 59.6 \\
10 & 49.5 & 49.6 \\
11 & 67.5 & 67.5 \\
12 & 41.9 & 41.9 \\
13 & 29.5 & 29.5 \\
14 & 44.3 & 44.4 \\
15 & 33.6 & 33.6 \\
16 & 145.6 & 145.6 \\
17 & 110.7 & 110.5 \\
18 & 70.8 & 70.8 \\
19 & 58.4 & 58.4 \\
20 & 75.9 & 75.9 \\
$1^{\prime}$ & 166.1 & 166.6 \\
$2^{\prime}$ & 130.1 & 130.1 \\
$3^{\prime}, 7^{\prime}$ & 129.6 & 129.5 \\
$4^{\prime}, 6^{\prime}$ & 128.5 & 128.4 \\
$5^{\prime}$ & 133.1 & 133.1 \\
\hline
\end{tabular}

*The synthetic sample was bubbled with a stream of $\mathrm{NH}_{3(\mathrm{~g})}$ for ca. $3 \mathrm{sec}$ in $\mathrm{CDCl}_{3}$ prior to $\mathrm{NMR}$ experiments. 


\section{II) Computational Details and Coordinates of Computed Stationary Points}

\section{Computational details:}

In the present studies, all geometries of reaction species were calculated by means of M06/6-31G(d,p) method, ${ }^{6-8}$ and frequency calculations were also employed to confirm the structures as minimum points in energy and achieve the relevant zero point energy (ZPE) in the gaseous phase. For each transition state, intrinsic reaction coordinate (IRC) calculations were performed to guarantee its correct connection to the designated local minima. In order to take the solvent effects into account, the conductor-like polarizable continuum model $(\mathrm{CPCM})^{9}$ was used for the single-point calculations based on the optimized geometries in the gas phase with the UAHF radii. Methanol was selected as the solvent according to the experimental conditions. All calculations were implemented via the Gaussian09 software package. ${ }^{10}$
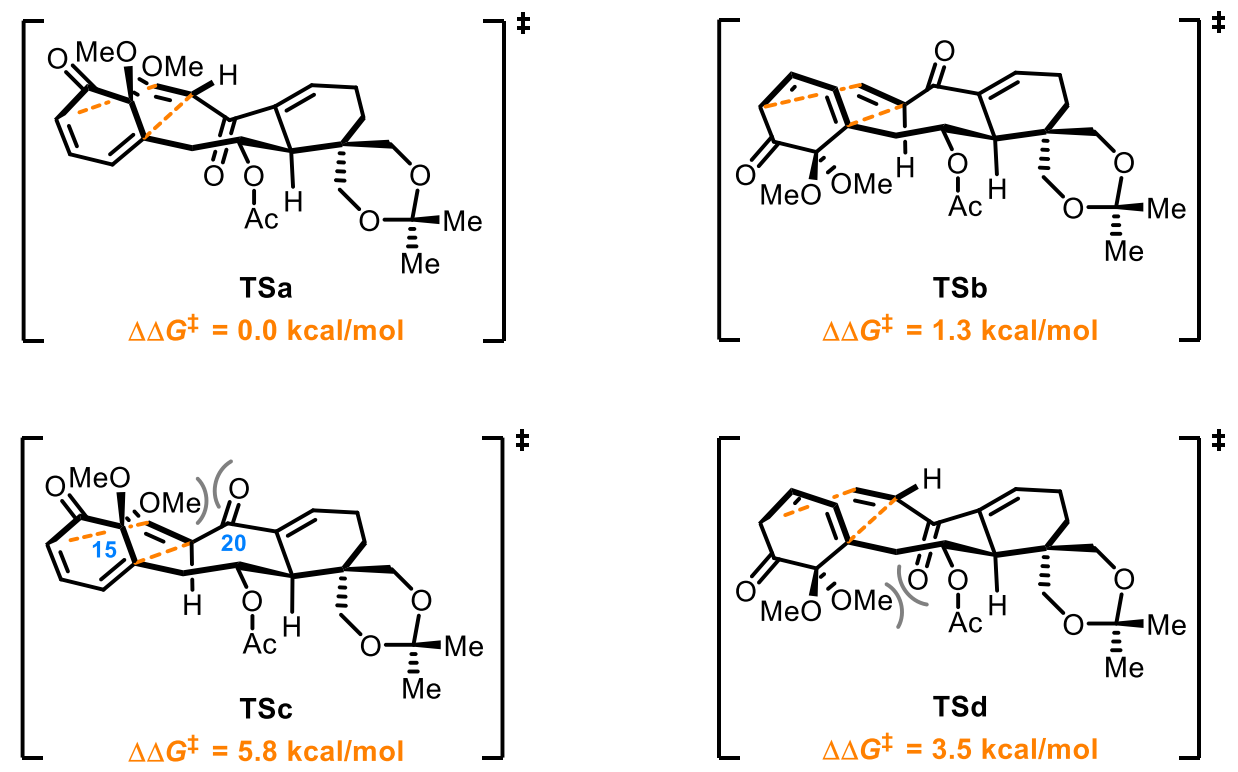

Scheme S1. Computed Free Energy Differences of the Four Transition States in the ODI-DielsAlder Cycloaddition of Vinylphenol 14. 
Cartesian coordinates and electronic energies for all of the calculated structures:

\section{TSa}

SCF done: $\quad-1611.951430$

\begin{tabular}{|c|c|c|c|}
\hline O & -4.713534 & -0.947288 & 0.748850 \\
\hline $\mathrm{O}$ & -4.501802 & 1.118090 & -0.319777 \\
\hline & 1.046625 & -3.504951 & 0.387130 \\
\hline & -0.142533 & 1.746421 & -0.016660 \\
\hline & -0.081463 & 3.061724 & -1.847533 \\
\hline D & 2.477966 & 1.465357 & 0.533132 \\
\hline & 3.922185 & 0.997165 & -1.198635 \\
\hline J & 4.989669 & 0.285767 & 1.466405 \\
\hline$C$ & -1.245611 & -2.760610 & -0.931193 \\
\hline $\mathrm{H}$ & -0.829670 & -3.767274 & -0.981000 \\
\hline $\mathrm{C}$ & -2.529563 & -2.479961 & -1.629526 \\
\hline $\mathrm{H}$ & -3.316155 & -3.090457 & -1.155067 \\
\hline $\mathrm{H}$ & -2.483298 & -2.847250 & -2.664087 \\
\hline C & -2.913771 & -1.007483 & -1.595390 \\
\hline $\mathrm{H}$ & -3.979944 & -0.879269 & -1.809335 \\
\hline $\mathrm{H}$ & -2.372225 & -0.468437 & -2.388408 \\
\hline $\mathrm{C}$ & -2.577821 & -0.357279 & -0.249409 \\
\hline $\mathrm{C}$ & -1.055997 & -0.446015 & -0.002081 \\
\hline $\mathrm{H}$ & -0.883258 & -0.154147 & 1.046314 \\
\hline $\mathrm{C}$ & -0.580357 & -1.8 & -0.180228 \\
\hline C & 0.658503 & -2.345073 & 0.508184 \\
\hline $\mathrm{C}$ & 2.706541 & -1.672421 & -1.291318 \\
\hline $\mathrm{H}$ & 2.172134 & -2.205474 & -2.076518 \\
\hline 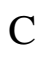 & 2.198156 & -0.472363 & -0.824384 \\
\hline C & 1.035521 & 0.164153 & -1.535564 \\
\hline $\mathrm{H}$ & 1.409444 & 1.062327 & -2.047863 \\
\hline$H$ & 0.721323 & -0.526356 & -2.330470 \\
\hline C & -0.276692 & 0.583077 & -0.852859 \\
\hline $\mathrm{H}$ & -0.900502 & 0.884986 & -1.705669 \\
\hline $\mathrm{C}$ & 3.730737 & -2.321348 & -0.606818 \\
\hline $\mathrm{H}$ & 4.012581 & -3.331877 & -0.890189 \\
\hline $\mathrm{C}$ & 4.230309 & -1.764661 & 0.568545 \\
\hline $\mathrm{H}$ & 5.015125 & -2.266190 & 1.132316 \\
\hline $\mathrm{C}$ & 1.456430 & -1.387025 & 1.283880 \\
\hline $\mathrm{H}$ & 1.094600 & -0.382772 & 1.484049 \\
\hline $\mathrm{C}$ & 3.179933 & 0.456101 & -0.115609 \\
\hline $\mathrm{C}$ & 4.209679 & -0.304890 & 0.740341 \\
\hline $\mathrm{C}$ & -3.316807 & -1.050156 & 0.902243 \\
\hline $\mathrm{H}$ & -3.093541 & -2.120487 & 0.959767 \\
\hline $\mathrm{H}$ & -2.982269 & -0.604885 & 1.857854 \\
\hline$\sigma$ & -3.093725 & 1.085234 & -0.22002 \\
\hline
\end{tabular}




$\begin{array}{lrrr}\mathrm{H} & -2.738780 & 1.588368 & 0.698101 \\ \mathrm{H} & -2.733098 & 1.674307 & -1.072989 \\ \mathrm{C} & -5.200497 & 0.380450 & 0.665206 \\ \mathrm{C} & -6.618257 & 0.264021 & 0.159370 \\ \mathrm{H} & -6.609898 & -0.247905 & -0.807536 \\ \mathrm{H} & -7.056505 & 1.258369 & 0.033428 \\ \mathrm{H} & -7.223531 & -0.313751 & 0.864145 \\ \mathrm{C} & -5.145976 & 1.077047 & 2.018709 \\ \mathrm{H} & -5.719866 & 0.499713 & 2.750516 \\ \mathrm{H} & -5.584982 & 2.075933 & 1.930272 \\ \mathrm{H} & -4.128115 & 1.191154 & 2.402845 \\ \mathrm{C} & -0.020408 & 2.929315 & -0.648175 \\ \mathrm{C} & 0.210696 & 4.042164 & 0.326324 \\ \mathrm{H} & -0.285542 & 3.850395 & 1.281309 \\ \mathrm{H} & -0.133676 & 4.982718 & -0.106790 \\ \mathrm{H} & 1.288772 & 4.122363 & 0.510401 \\ \mathrm{C} & 2.736060 & 1.875648 & 1.860391 \\ \mathrm{H} & 3.663210 & 2.452998 & 1.951673 \\ \mathrm{H} & 2.788406 & 1.036815 & 2.565654 \\ \mathrm{H} & 1.877110 & 2.497795 & 2.135293 \\ \mathrm{C} & 4.718824 & 2.129304 & -0.900606 \\ \mathrm{H} & 5.202044 & 2.409606 & -1.839307 \\ \mathrm{H} & 5.484656 & 1.909291 & -0.146014 \\ \mathrm{H} & 4.100982 & 2.967734 & -0.553806 \\ \mathrm{C} & 2.587485 & -1.876252 & 1.924414 \\ \mathrm{H} & 2.685939 & -2.957484 & 1.981277 \\ \mathrm{H} & 3.061255 & -1.303960 & 2.720025\end{array}$

\section{TSb}

SCF done: -1611.948128

$\begin{array}{lrcc}\mathrm{O} & 4.690674 & -1.441674 & -0.099887 \\ \mathrm{O} & 4.669495 & 0.839493 & 0.403213 \\ \mathrm{O} & -0.451903 & -2.118170 & -1.861642 \\ \mathrm{O} & 0.545239 & 1.945478 & -0.669352 \\ \mathrm{O} & 0.655520 & 3.575623 & 0.888255 \\ \mathrm{C} & 0.722781 & -2.305134 & 1.348746 \\ \mathrm{H} & 0.157054 & -3.211566 & 1.575397 \\ \mathrm{C} & 1.931314 & -2.024664 & 2.183461 \\ \mathrm{H} & 2.646944 & -2.849717 & 2.032981 \\ \mathrm{H} & 1.676330 & -2.068287 & 3.251368 \\ \mathrm{C} & 2.595951 & -0.691851 & 1.853239 \\ \mathrm{H} & 3.632811 & -0.686091 & 2.205776 \\ \mathrm{H} & 2.085734 & 0.116478 & 2.398668 \\ \mathrm{C} & 2.563810 & -0.365306 & 0.354705 \\ \mathrm{C} & 1.103359 & -0.321759 & -0.128541\end{array}$




\begin{tabular}{|c|c|c|c|}
\hline $\mathrm{H}$ & 1.095274 & -0.296269 & -1.229540 \\
\hline $\mathrm{C}$ & 0.376201 & -1.579030 & 0.280235 \\
\hline $\mathrm{C}$ & -0.695978 & -2.031899 & -0.656994 \\
\hline $\mathrm{C}$ & -2.120379 & 0.329219 & -0.190188 \\
\hline $\mathrm{C}$ & -1.094044 & 0.836263 & 0.775454 \\
\hline $\mathrm{H}$ & -1.403159 & 1.832341 & 1.129786 \\
\hline $\mathrm{H}$ & -1.103343 & 0.193453 & 1.666484 \\
\hline $\mathrm{C}$ & 0.369930 & 0.948224 & 0.359932 \\
\hline $\mathrm{H}$ & 0.874498 & 1.332314 & 1.256481 \\
\hline $\mathrm{C}$ & -3.880694 & -1.042997 & -1.876544 \\
\hline $\mathrm{C}$ & -2.023901 & -2.267937 & -0.124308 \\
\hline $\mathrm{H}$ & -2.213437 & -2.154761 & 0.939426 \\
\hline $\mathrm{C}$ & 3.326386 & -1.417593 & -0.457441 \\
\hline $\mathrm{H}$ & 2.944083 & -2.429813 & -0.288600 \\
\hline $\mathrm{H}$ & 3.193186 & -1.207750 & -1.534369 \\
\hline $\mathrm{C}$ & 3.299338 & 0.949115 & 0.083640 \\
\hline $\mathrm{H}$ & 3.150673 & 1.242218 & -0.972862 \\
\hline $\mathrm{H}$ & 2.917237 & 1.769931 & 0.705228 \\
\hline $\mathrm{C}$ & 5.362057 & -0.205669 & -0.256354 \\
\hline $\mathrm{C}$ & 6.670420 & -0.356071 & 0.482328 \\
\hline $\mathrm{H}$ & 6.461784 & -0.594801 & 1.529509 \\
\hline $\mathrm{H}$ & 7.239967 & 0.576727 & 0.434809 \\
\hline $\mathrm{H}$ & 7.259892 & -1.165414 & 0.041395 \\
\hline $\mathrm{C}$ & 5.586903 & 0.122771 & -1.726727 \\
\hline $\mathrm{H}$ & 6.153153 & -0.686308 & -2.199028 \\
\hline $\mathrm{H}$ & 6.159725 & 1.052119 & -1.808155 \\
\hline $\mathrm{H}$ & 4.655847 & 0.251178 & -2.286663 \\
\hline $\mathrm{C}$ & 0.699962 & 3.224865 & -0.265717 \\
\hline $\mathrm{C}$ & 0.967241 & 4.111056 & -1.441823 \\
\hline $\mathrm{H}$ & 1.924484 & 3.829353 & -1.894239 \\
\hline $\mathrm{H}$ & 1.004988 & 5.151475 & -1.118592 \\
\hline $\mathrm{H}$ & 0.195854 & 3.977498 & -2.205603 \\
\hline $\mathrm{C}$ & -1.950293 & 0.372860 & -1.556452 \\
\hline $\mathrm{H}$ & -1.025812 & 0.770399 & -1.966306 \\
\hline $\mathrm{C}$ & -3.553316 & 0.225176 & 0.340668 \\
\hline $\mathrm{O}$ & -3.979020 & 1.566182 & 0.330858 \\
\hline $\mathrm{O}$ & -3.701201 & -0.376637 & 1.597294 \\
\hline $\mathrm{C}$ & -5.336633 & 1.825973 & 0.667363 \\
\hline $\mathrm{H}$ & -5.365953 & 2.859984 & 1.022256 \\
\hline $\mathrm{H}$ & -5.993306 & 1.722498 & -0.203857 \\
\hline $\mathrm{H}$ & -5.698833 & 1.151326 & 1.449649 \\
\hline $\mathrm{C}$ & -3.447663 & 0.366041 & 2.773682 \\
\hline $\mathrm{H}$ & -2.512362 & 0.042897 & 3.248866 \\
\hline $\mathrm{H}$ & -3.393916 & 1.442461 & 2.581549 \\
\hline $\mathrm{H}$ & -4.271359 & 0.176464 & 3.470779 \\
\hline
\end{tabular}




$\begin{array}{llll}\mathrm{C} & -4.468407 & -0.605979 & -0.595438 \\ \mathrm{O} & -5.611605 & -0.855771 & -0.279353 \\ \mathrm{H} & -4.592738 & -1.535468 & -2.537558 \\ \mathrm{C} & -2.836066 & -0.287043 & -2.411523 \\ \mathrm{H} & -2.608461 & -0.348888 & -3.472345 \\ \mathrm{C} & -3.024474 & -2.671039 & -1.004916 \\ \mathrm{H} & -3.953916 & -3.073536 & -0.605005 \\ \mathrm{H} & -2.693368 & -3.084167 & -1.955699\end{array}$

\section{TSc}

SCF done: -1611.940175

$\begin{array}{lccc}\mathrm{O} & -0.068995 & 1.819656 & -0.126100 \\ \mathrm{O} & 0.002487 & 3.141464 & -1.952584 \\ \mathrm{O} & 1.692414 & 0.202665 & 1.242986 \\ \mathrm{O} & 2.850671 & 1.835424 & 0.207055 \\ \mathrm{O} & 4.498619 & 0.240438 & 1.999552 \\ \mathrm{C} & -0.942678 & -2.685205 & -1.136504 \\ \mathrm{H} & -0.501332 & -3.680727 & -1.225834 \\ \mathrm{C} & -2.239904 & -2.450878 & -1.844443 \\ \mathrm{H} & -2.981809 & -3.150224 & -1.424544 \\ \mathrm{H} & -2.157667 & -2.732251 & -2.903504 \\ \mathrm{C} & -2.747599 & -1.018304 & -1.715860 \\ \mathrm{H} & -3.824871 & -0.977286 & -1.910044 \\ \mathrm{H} & -2.275520 & -0.391768 & -2.488382 \\ \mathrm{C} & -2.450354 & -0.404384 & -0.341933 \\ \mathrm{C} & -0.931343 & -0.410187 & -0.095469 \\ \mathrm{H} & -0.737158 & -0.145306 & 0.954392 \\ \mathrm{C} & -0.380355 & -1.799297 & -0.307858 \\ \mathrm{C} & 0.756763 & -2.189823 & 0.587390 \\ \mathrm{C} & 3.390132 & -0.651057 & -1.763537 \\ \mathrm{H} & 3.221845 & -0.756156 & -2.836146 \\ \mathrm{C} & 2.356914 & -0.176923 & -0.969432 \\ \mathrm{C} & 1.101371 & 0.279232 & -1.663538 \\ \mathrm{H} & 1.365622 & 1.166996 & -2.259604 \\ \mathrm{H} & 0.836044 & -0.493080 & -2.400432 \\ \mathrm{C} & -0.202152 & 0.648971 & -0.952236 \\ \mathrm{H} & -0.854527 & 0.935778 & -1.789854 \\ \mathrm{C} & 4.556453 & -1.166111 & -1.197322 \\ \mathrm{H} & 5.303707 & -1.637755 & -1.831611 \\ \mathrm{C} & 4.642963 & -1.274630 & 0.192434 \\ \mathrm{H} & 5.539588 & -1.684208 & 0.656181 \\ \mathrm{C} & 2.731284 & 0.435102 & 0.371468 \\ \mathrm{H} & -3.026568 & -0.178156 & 0.960875 \\ \mathrm{H} & -2.862989 & -2.252977 & 0.770617\end{array}$




\begin{tabular}{|c|c|c|c|}
\hline $\mathrm{H}$ & -2.805138 & -0.792375 & 1.754065 \\
\hline $\mathrm{C}$ & -3.037834 & 1.005761 & -0.249275 \\
\hline $\mathrm{H}$ & -2.690512 & 1.488051 & 0.683204 \\
\hline $\mathrm{H}$ & -2.714012 & 1.643467 & -1.082613 \\
\hline $\mathrm{C}$ & -5.085781 & 0.162191 & 0.641276 \\
\hline $\mathrm{C}$ & -6.510613 & 0.010286 & 0.165282 \\
\hline $\mathrm{H}$ & -6.505431 & -0.451770 & -0.826470 \\
\hline $\mathrm{H}$ & -6.992716 & 0.990218 & 0.100877 \\
\hline $\mathrm{H}$ & -7.072725 & -0.627265 & 0.854064 \\
\hline $\mathrm{C}$ & -5.023219 & 0.795493 & 2.025097 \\
\hline $\mathrm{H}$ & -5.567300 & 0.168390 & 2.738468 \\
\hline $\mathrm{H}$ & -5.489296 & 1.785563 & 1.993813 \\
\hline $\mathrm{H}$ & -4.001185 & 0.915411 & 2.395706 \\
\hline $\mathrm{C}$ & 0.039254 & 3.004697 & -0.751367 \\
\hline $\mathrm{C}$ & 0.224121 & 4.106978 & 0.243942 \\
\hline $\mathrm{H}$ & 0.105509 & 5.073370 & -0.247436 \\
\hline $\mathrm{H}$ & 1.232024 & 4.028601 & 0.666702 \\
\hline $\mathrm{H}$ & -0.486921 & 4.005020 & 1.069084 \\
\hline $\mathrm{C}$ & 1.628003 & 0.916325 & 2.466483 \\
\hline $\mathrm{H}$ & 0.945435 & 0.338416 & 3.096720 \\
\hline $\mathrm{H}$ & 1.219420 & 1.919052 & 2.297172 \\
\hline $\mathrm{H}$ & 2.606242 & 0.986453 & 2.949466 \\
\hline $\mathrm{C}$ & 3.972677 & 2.321485 & -0.493832 \\
\hline $\mathrm{H}$ & 3.914808 & 3.411928 & -0.447870 \\
\hline $\mathrm{H}$ & 3.967275 & 2.014692 & -1.550295 \\
\hline $\mathrm{H}$ & 4.919061 & 2.000929 & -0.035935 \\
\hline $\mathrm{C}$ & 2.038150 & -2.506043 & -0.059393 \\
\hline $\mathrm{H}$ & 1.981668 & -2.808484 & -1.103754 \\
\hline $\mathrm{C}$ & 3.195364 & -2.730286 & 0.673342 \\
\hline $\mathrm{H}$ & 3.908052 & -3.482560 & 0.349579 \\
\hline $\mathrm{H}$ & 3.123293 & -2.583217 & 1.752455 \\
\hline
\end{tabular}

\section{TSd}

SCF done: -1611.9468072

$\begin{array}{lrrr}\mathrm{O} & -4.543072 & -1.469968 & 0.591905 \\ \mathrm{O} & -4.664149 & 0.667216 & -0.335863 \\ \mathrm{O} & 1.489660 & -3.092116 & 0.370508 \\ \mathrm{O} & -0.627902 & 2.055627 & 0.063273 \\ \mathrm{O} & -0.850621 & 3.308087 & -1.802367 \\ \mathrm{C} & -0.768318 & -2.540844 & -1.062358 \\ \mathrm{H} & -0.199322 & -3.466323 & -1.150901 \\ \mathrm{C} & -2.043806 & -2.418656 & -1.817163 \\ \mathrm{H} & -2.725455 & -3.202992 & -1.446529 \\ \mathrm{H} & -1.883044 & -2.672838 & -2.874002 \\ \mathrm{C} & -2.693531 & -1.049853 & -1.683192\end{array}$




\begin{tabular}{|c|c|c|c|}
\hline $\mathrm{H}$ & -3.760867 & -1.103825 & -1.921186 \\
\hline $\mathrm{H}$ & -2.253876 & -0.359082 & -2.417786 \\
\hline $\mathrm{C}$ & -2.511532 & -0.454608 & -0.283177 \\
\hline $\mathrm{C}$ & -1.002936 & -0.305546 & 0.033583 \\
\hline $\mathrm{H}$ & -0.924471 & -0.043001 & 1.100742 \\
\hline $\mathrm{C}$ & -0.291508 & -1.627128 & -0.202631 \\
\hline $\mathrm{C}$ & 0.946429 & -2.012001 & 0.556357 \\
\hline $\mathrm{C}$ & 2.131392 & 0.691212 & -0.002201 \\
\hline $\mathrm{C}$ & 1.105078 & 0.796403 & -1.095075 \\
\hline $\mathrm{H}$ & 1.332070 & 1.691766 & -1.694900 \\
\hline $\mathrm{H}$ & 1.235390 & -0.051692 & -1.776349 \\
\hline $\mathrm{C}$ & -0.369260 & 0.874324 & -0.732993 \\
\hline $\mathrm{H}$ & -0.892469 & 1.008184 & -1.689350 \\
\hline $\mathrm{C}$ & 3.968939 & 0.259033 & 2.047242 \\
\hline $\mathrm{C}$ & 1.441198 & -1.072542 & 1.582565 \\
\hline $\mathrm{H}$ & 0.732558 & -0.358970 & 1.992922 \\
\hline $\mathrm{C}$ & -3.153689 & -1.346700 & 0.787590 \\
\hline $\mathrm{H}$ & -2.752300 & -2.365027 & 0.777034 \\
\hline $\mathrm{H}$ & -2.924921 & -0.926302 & 1.784851 \\
\hline $\mathrm{C}$ & -3.274465 & 0.871849 & -0.189666 \\
\hline $\mathrm{H}$ & -3.038570 & 1.374244 & 0.766647 \\
\hline $\mathrm{H}$ & -3.001963 & 1.558046 & -1.001877 \\
\hline $\mathrm{C}$ & -5.246174 & -0.241260 & 0.579508 \\
\hline $\mathrm{C}$ & -6.614756 & -0.558196 & 0.026171 \\
\hline $\mathrm{H}$ & -6.503411 & -0.990388 & -0.972727 \\
\hline $\mathrm{H}$ & -7.214015 & 0.354387 & -0.043811 \\
\hline $\mathrm{H}$ & -7.123963 & -1.279054 & 0.672680 \\
\hline $\mathrm{C}$ & -5.336290 & 0.361571 & 1.975960 \\
\hline $\mathrm{H}$ & -5.818173 & -0.352350 & 2.651554 \\
\hline $\mathrm{H}$ & -5.936536 & 1.276317 & 1.939281 \\
\hline $\mathrm{H}$ & -4.360546 & 0.618186 & 2.399652 \\
\hline $\mathrm{C}$ & -0.857454 & 3.209004 & -0.600087 \\
\hline $\mathrm{C}$ & -1.142888 & 4.316295 & 0.367281 \\
\hline $\mathrm{H}$ & -2.059594 & 4.089323 & 0.922419 \\
\hline $\mathrm{H}$ & -1.262739 & 5.255716 & -0.172830 \\
\hline $\mathrm{H}$ & -0.335478 & 4.404306 & 1.101392 \\
\hline $\mathrm{C}$ & 2.230459 & 1.597913 & 1.043622 \\
\hline $\mathrm{H}$ & 1.467606 & 2.365785 & 1.156403 \\
\hline $\mathrm{C}$ & 3.405589 & -0.032914 & -0.432958 \\
\hline $\mathrm{O}$ & 4.027746 & 0.735802 & -1.450263 \\
\hline $\mathrm{O}$ & 3.021132 & -1.212898 & -1.027047 \\
\hline $\mathrm{C}$ & 4.704680 & 1.913278 & -1.079803 \\
\hline $\mathrm{H}$ & 5.145871 & 2.314271 & -1.995372 \\
\hline $\mathrm{H}$ & 4.024233 & 2.668175 & -0.657001 \\
\hline $\mathrm{H}$ & 5.511737 & 1.721110 & -0.358055 \\
\hline
\end{tabular}




$\begin{array}{lrrr}\mathrm{C} & 4.005026 & -2.135204 & -1.464582 \\ \mathrm{H} & 4.811345 & -1.632213 & -2.007267 \\ \mathrm{H} & 4.427526 & -2.698616 & -0.625152 \\ \mathrm{H} & 3.476813 & -2.825450 & -2.126755 \\ \mathrm{C} & 4.387825 & -0.262660 & 0.738483 \\ \mathrm{O} & 5.453629 & -0.817549 & 0.557107 \\ \mathrm{H} & 4.691572 & 0.084564 & 2.843521 \\ \mathrm{C} & 3.171409 & 1.404545 & 2.056075 \\ \mathrm{H} & 3.163727 & 2.051947 & 2.930379 \\ \mathrm{C} & 2.589175 & -1.338271 & 2.314536 \\ \mathrm{H} & 3.193012 & -2.192407 & 2.002143 \\ \mathrm{H} & 2.623307 & -1.103678 & 3.373684\end{array}$




\section{III) Abbreviations}

\begin{tabular}{|c|c|}
\hline Ac & acetyl \\
\hline acac & acetylacetonate \\
\hline AIBN & 2,2'-azobis(iso-butyronitrile) \\
\hline $\mathrm{Bz}$ & benzoyl \\
\hline $\mathrm{Cp}$ & cyclopentadienyl \\
\hline DCE & 1,2-dichloroethane \\
\hline DDQ & 2,3-dichloro-5,6-dicyano-1,4-benzoquinone \\
\hline DIBAL-H & di-iso-butyl aluminium hydride \\
\hline dibm & 2,6-dimethyl-3,5-heptanedionate \\
\hline 4-DMAP & 4-dimethylaminopyridine \\
\hline DMF & $N, N^{\prime}$-dimethylformamide \\
\hline 2,2-DMP & 2,2-dimethoxypropane \\
\hline dpm & 2,2,6,6-dimethyl-3,5-heptanedionate \\
\hline LDA & Lithium di-iso-propylamide \\
\hline L-Selectride & Lithium tri-sec-butylborohydride \\
\hline Mes & 2,4,6-trimethylphenyl \\
\hline MS & molecular sieves \\
\hline NCS & $N$-chlorosuccinimide \\
\hline NMO & $N$-methylmorpholine $N$-oxide \\
\hline $\mathrm{Pc}$ & phthalocyanine \\
\hline PMB & $p$-methoxybenzyl \\
\hline
\end{tabular}




$\begin{array}{ll}\text { PPTS } & \text { pyridinium 4-toluenesulfonate } \\ \text { TBACl } & \text { tetra- } n \text {-butylammonium chloride } \\ \text { TBAF } & \text { tetra- } n \text {-butylammonium fluoride } \\ \text { TBHP } & \text { tert-butyl hydroperoxide } \\ \text { TBSOTf } & \text { tert-butyldimethylsilyl trifluoromethanesulfonate } \\ \text { TCDI } & 1,1^{\prime} \text {-thiocarbonyldiimidazole } \\ \text { TEMPO } & 2,2,6,6 \text {-tetramethyl-1-piperidinyloxy } \\ \text { tfa } & 1,1,1 \text {-trifluoropentane-2,4-dionato } \\ \text { TFA } & \text { trifluoroacetic acid } \\ \text { THF } & \text { tetrahydrofuran } \\ \text { TMDSO } & \text { tetramethyldisiloxane } \\ & \text { tetra- } n \text {-propylammonium perruthenate }\end{array}$

\section{IV) References}

(1) For preparation, see: Yu, K.; Yang, Z.-N.; Liu, C.-H.; Wu, S.-Q.; Hong, X.; Zhao, X.-L.; Ding, H. Angew. Chem., Int. Ed. 2019, 58, 8556-8560.

(2) Alternatively, $\mathbf{1 5}$ could also be prepared according to the following procedure: Biswas, G.; Ghorai, S.; Bhattacharjya, A. Org. Lett. 2006, 8, 313-316.

(3) For an analogous preparation procedure, see: Birman, V. B.; Li, X. Org. Lett. 2008, 10, $1115-1118$.

(4) For preparation of Co(Salen) complexes, see: (a) Ford, D. D.; Nielsen, L. P. C.; Zuend, S. J.; Musgrave, C. B.; Jacobsen, E. N. J. Am. Chem. Soc. 2013, 135, 15595-15608. (b) Schaus, S. E.; 
Brandes, B. D.; Larrow, J. F.; Tokunaga, M.; Hansen, K. B.; Gould, A. E.; Furrow, M. E.; Jacobsen, E. N. J. Am. Chem. Soc. 2002, 124, 1307-1315.

(5) Ulubelen, A.; Desai, H. K.; Srivastava, S. K.; Hart, B. P.; Park, J.-C.; Joshi, B. S.; Pelletier, S. W.; Meriçli, A. H.; Meriçli, F.; Ilarslan, R. J. Nat. Prod. 1996, 59, 360-366.

(6) Zhao, Y.; Truhlar, D. G. Theor. Chem. Acc. 2008, 120, 215-241.

(7) Zhao, Y.; Truhlar, D. G. J. Phys. Chem. A. 2006, 110, 5121-5129.

(8) Zhao, Y.; Truhlar, D. G. J. Phys. Chem. A. 2006, 110, 13126-13130.

(9) (a) Barone, V.; Cossi, M. J. Phys. Chem. A. 1998, 102, 1995-2001. (b) Cossi, M.; Rega, N.; Scalmani, G.; Barone, V. J. Comput. Chem. 2003, 24, 669-681.

(10)Gaussian 09, Revision A.1, Frisch, M. J.; Trucks, G. W.; Schlegel, H. B.; Scuseria, G. E.; Robb, M. A.; Cheeseman, J. R.; Scalmani, G.; Barone, V.; Mennucci, B.; Petersson, G. A.; Nakatsuji, H.; Caricato, M.; Li, X.; Hratchian, H. P.; Izmaylov, A. F.; Bloino, J.; Zheng, G.; Sonnenberg, J. L.; Hada, M.; Ehara, M.; Toyota, K.; Fukuda, R.; Hasegawa, J.; Ishida, M.; Nakajima, T.; Honda, Y.; Kitao, O.; Nakai, H.; Vreven, T.; Montgomery, J. A., Jr.; Peralta, J. E.; Ogliaro, F.; Bearpark, M.; Heyd, J. J.; Brothers, E.; Kudin, K. N.; Staroverov, V. N.; Kobayashi, R.; Normand, J.; Raghavachari, K.; Rendell, A.; Burant, J. C.; Iyengar, S. S.; Tomasi, J.; Cossi, M.; Rega, N.; Millam, J. M.; Klene, M.; Knox, J. E.; Cross, J. B.; Bakken, V.; Adamo, C.; Jaramillo, J.; Gomperts, R.; Stratmann, R. E.; Yazyev, O.; Austin, A. J.; Cammi, R.; Pomelli, C.; Ochterski, J. W.; Martin, R. L.; Morokuma, K.; Zakrzewski, V. G.; Voth, G. A.; Salvador, P.; Dannenberg, J. J.; Dapprich, S.; Daniels, A. D.; Farkas, Ö.; Foresman, J. B.; Ortiz, J. V.; Cioslowski, J.; Fox, D. J. Gaussian, Inc., Wallingford CT, 2009. 
V) ${ }^{1} \mathrm{H}$ and ${ }^{13} \mathrm{C}$ NMR Spectra of Compounds

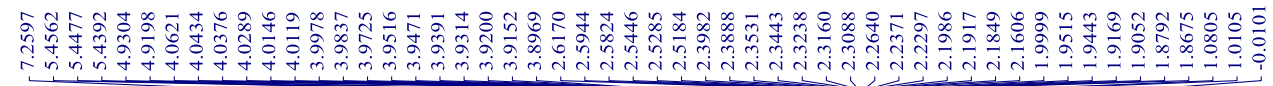

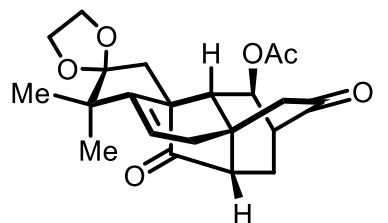

11'

${ }^{1} \mathrm{H}$ NMR spectrum $\left(400 \mathrm{MHz}, \mathrm{CDCl}_{3}\right)$

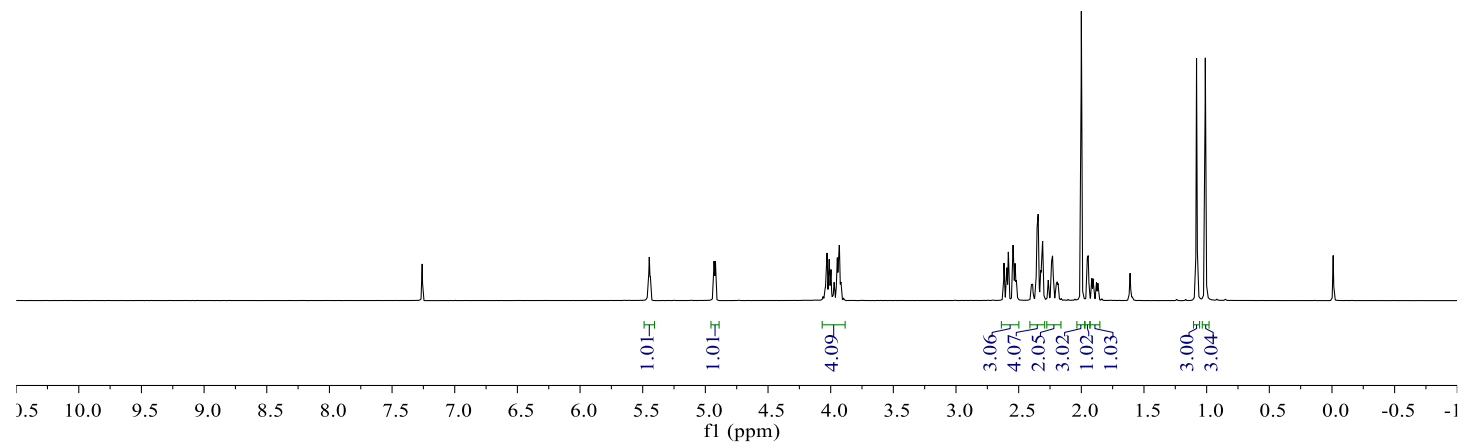

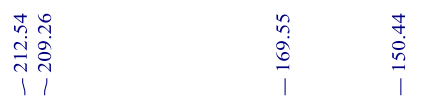

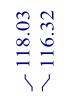

$\begin{array}{ll}1 & 1 \\ 0 & 1\end{array}$

${ }^{13} \mathrm{C}$ NMR spectrum $\left(100 \mathrm{MHz}, \mathrm{CDCl}_{3}\right)$

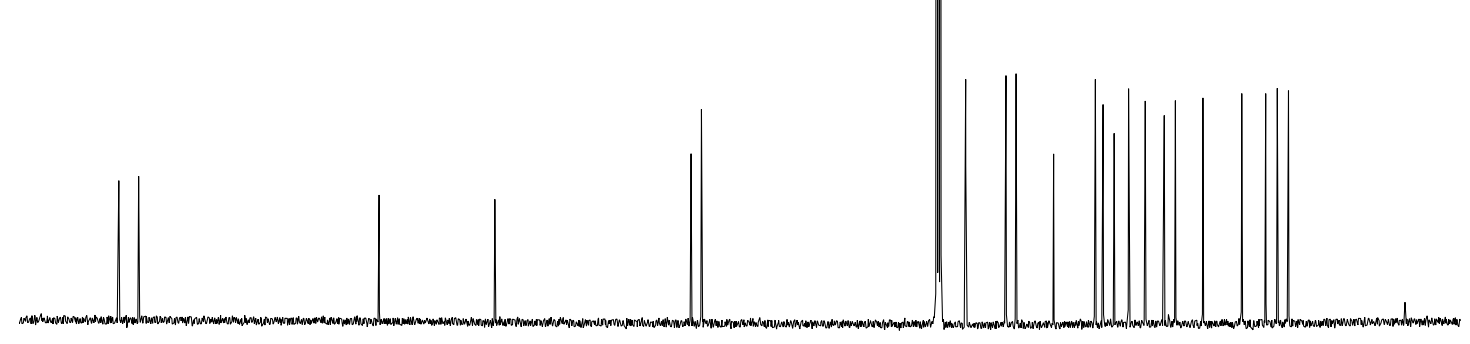

$\begin{array}{lllllllllllll}220 & 210 & 200 & 190 & 180 & 170 & 160 & 150 & 140 & 130 & 120 & 110 & 100\end{array}$ 


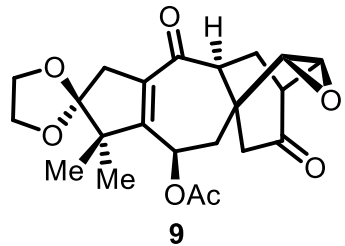

${ }^{1} \mathrm{H}$ NMR spectrum $\left(400 \mathrm{MHz}, \mathrm{CDCl}_{3}\right)$

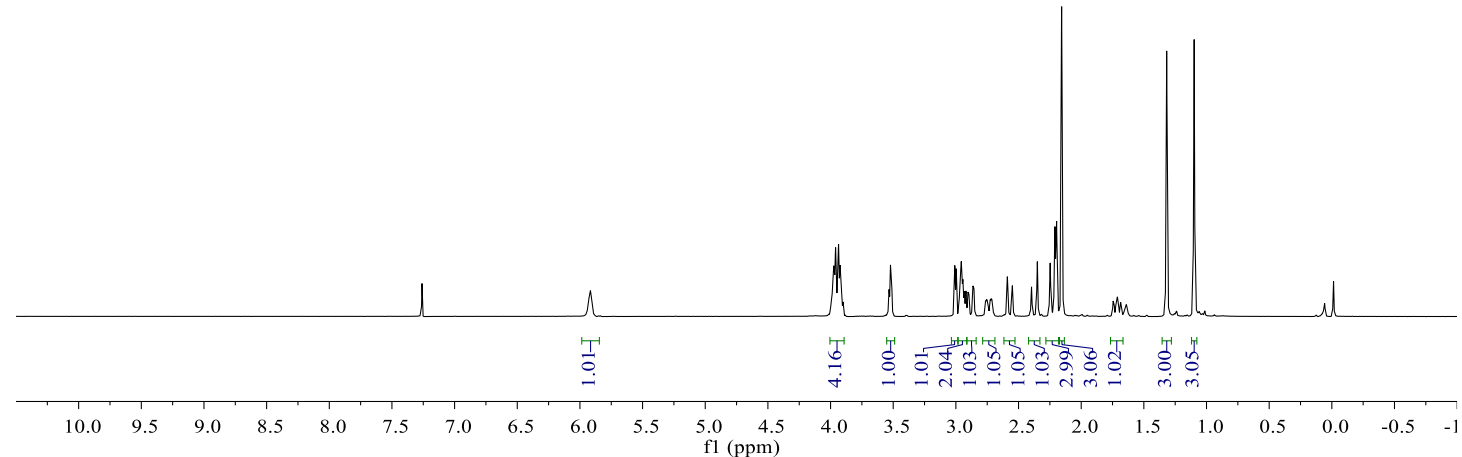

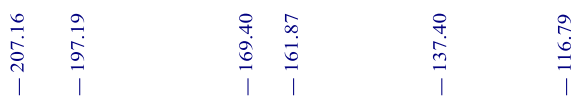

${ }^{13} \mathrm{C}$ NMR spectrum $\left(100 \mathrm{MHz}, \mathrm{CDCl}_{3}\right)$

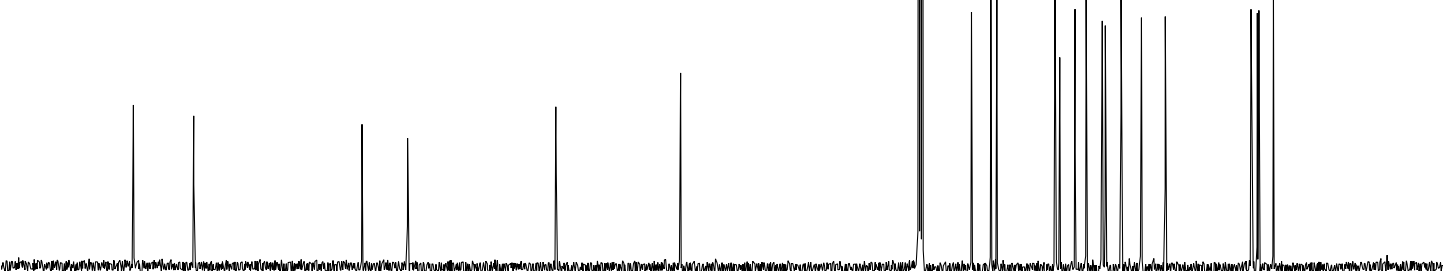

$\begin{array}{llllllllllllllllllllllllllllll}220 & 210 & 200 & 190 & 180 & 170 & 160 & 150 & 140 & 130 & 120 & 110 & 100 & 90 & 80 & 70 & 60 & 50 & 40 & 30 & 20 & 10 & 0\end{array}$ 

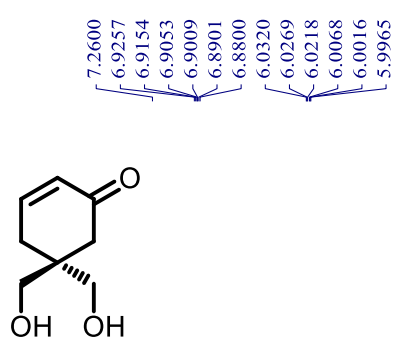

S-1

${ }^{1} \mathrm{H}$ NMR spectrum (400 MHz, $\mathrm{CDCl}_{3}$ )

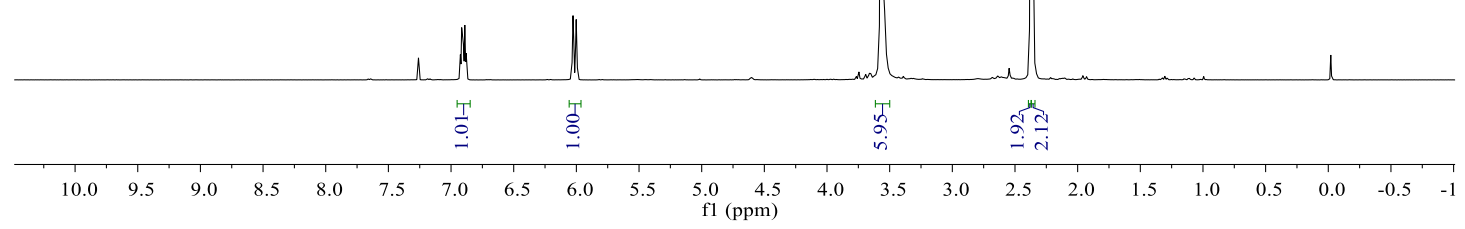

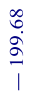

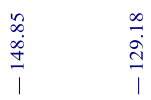

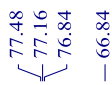

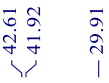

${ }^{13} \mathrm{C}$ NMR spectrum $\left(100 \mathrm{MHz}, \mathrm{CDCl}_{3}\right)$

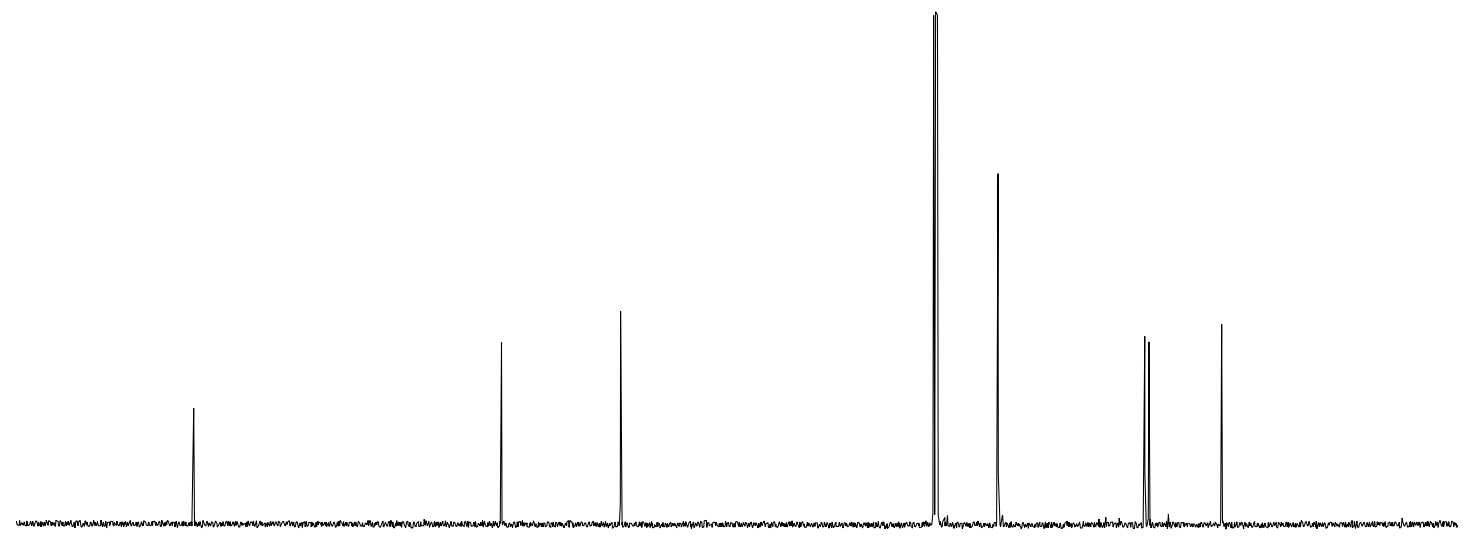

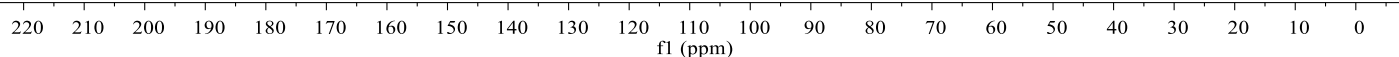




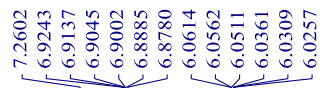

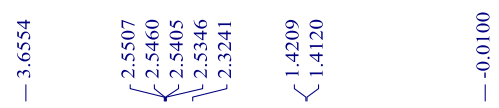

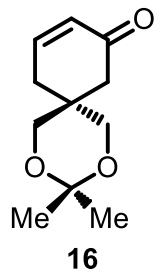

${ }^{1} \mathrm{H}$ NMR spectrum $\left(400 \mathrm{MHz}, \mathrm{CDCl}_{3}\right)$

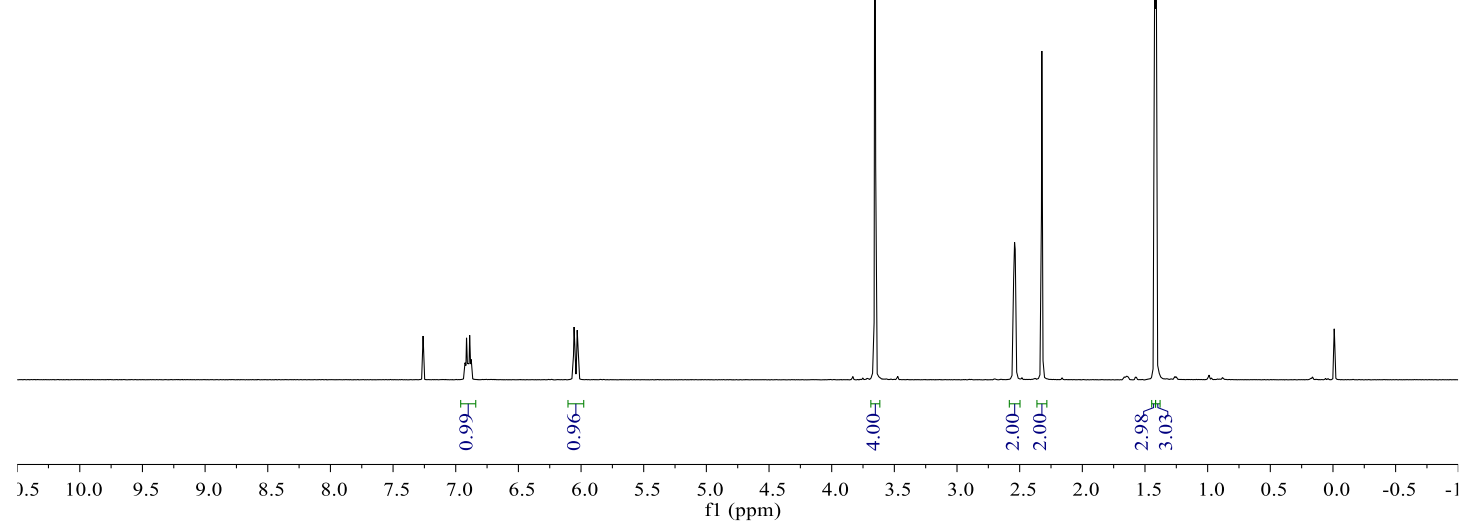

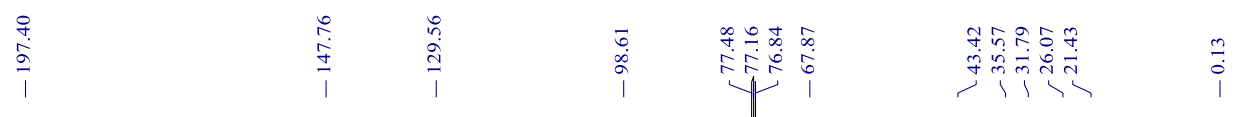

${ }^{13} \mathrm{C}$ NMR spectrum $\left(100 \mathrm{MHz}, \mathrm{CDCl}_{3}\right)$

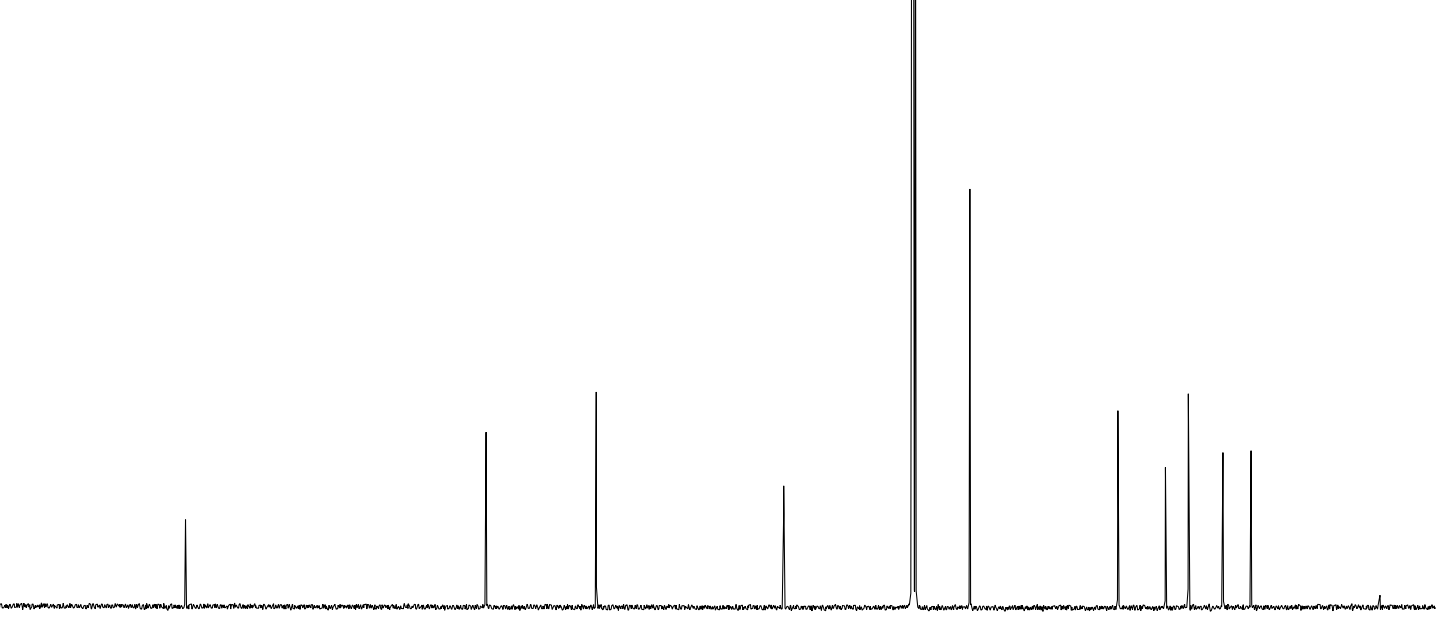

$\begin{array}{lllllllllllllllllllllll}220 & 210 & 200 & 190 & 180 & 170 & 160 & 150 & 140 & 130 & 120 & 110 & 100 & 90 & 80 & 70 & 60 & 50 & 40 & 30 & 20 & 10 & 0\end{array}$ 


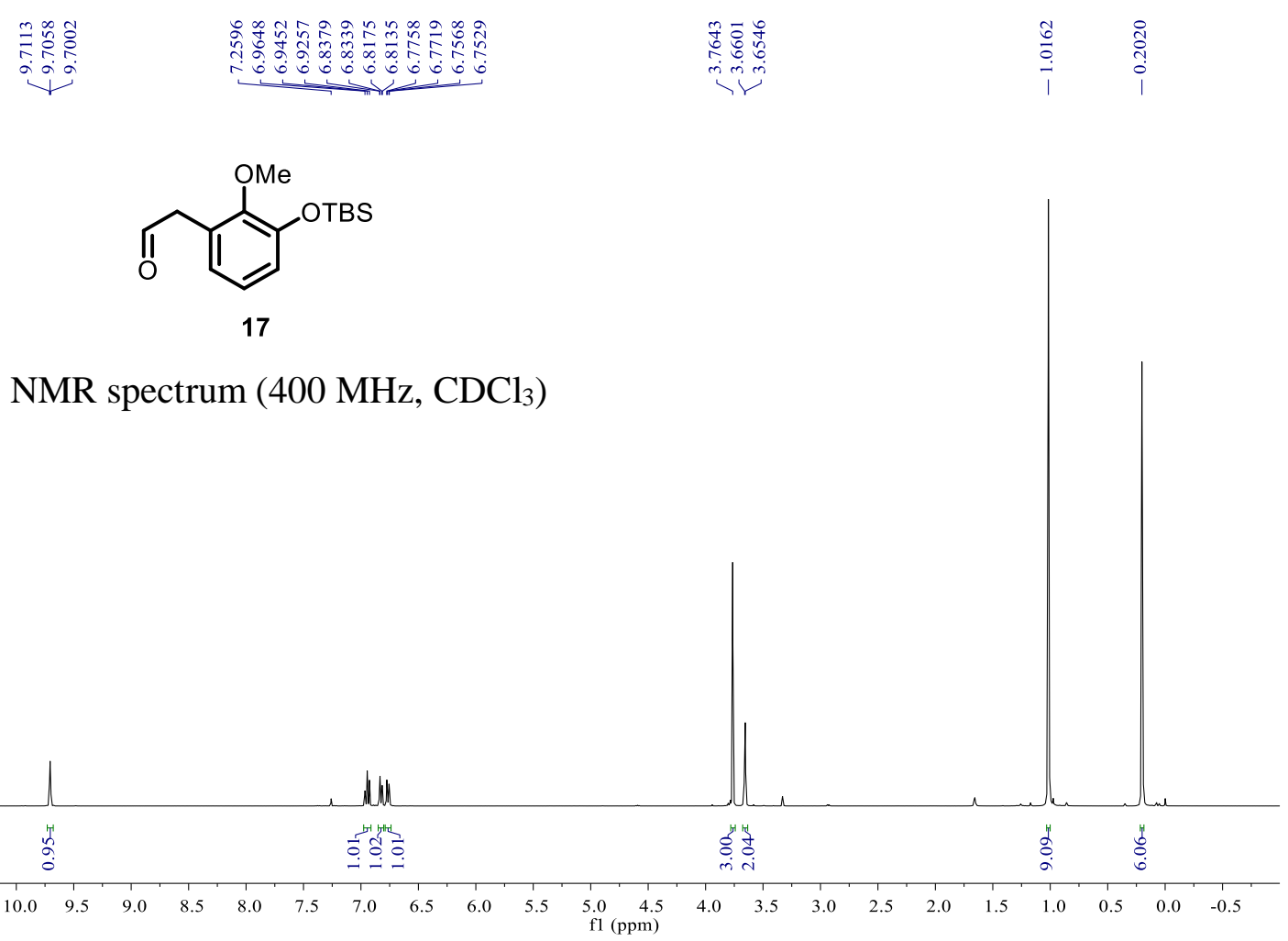

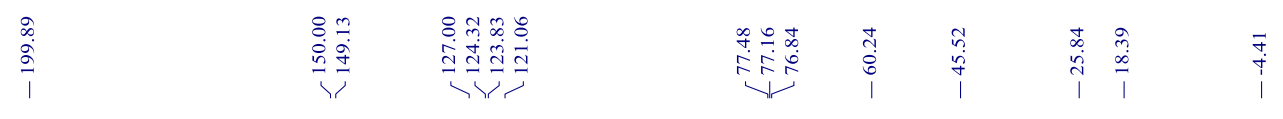

${ }^{13} \mathrm{C}$ NMR spectrum $\left(100 \mathrm{MHz}, \mathrm{CDCl}_{3}\right)$

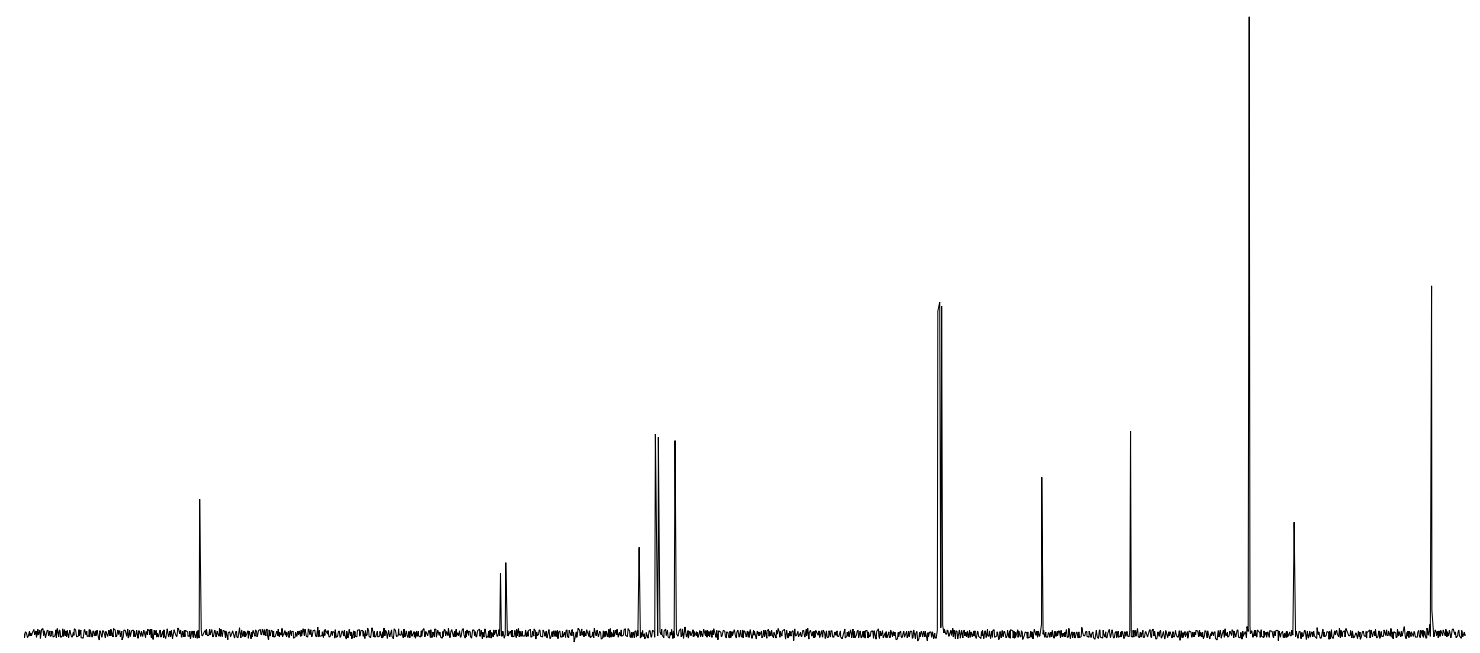

$\begin{array}{lllllllllllllllllllllllllllll}220 & 210 & 200 & 190 & 180 & 170 & 160 & 150 & 140 & 130 & 120 & 110 & 100 & 90 & 80 & 70 & 60 & 50 & 40 & 30 & 20 & 10 & 0 & -\end{array}$ 


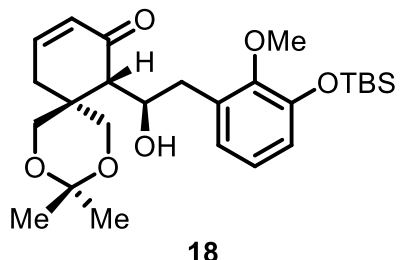

${ }^{1} \mathrm{H}$ NMR spectrum $\left(400 \mathrm{MHz}, \mathrm{CDCl}_{3}\right)$

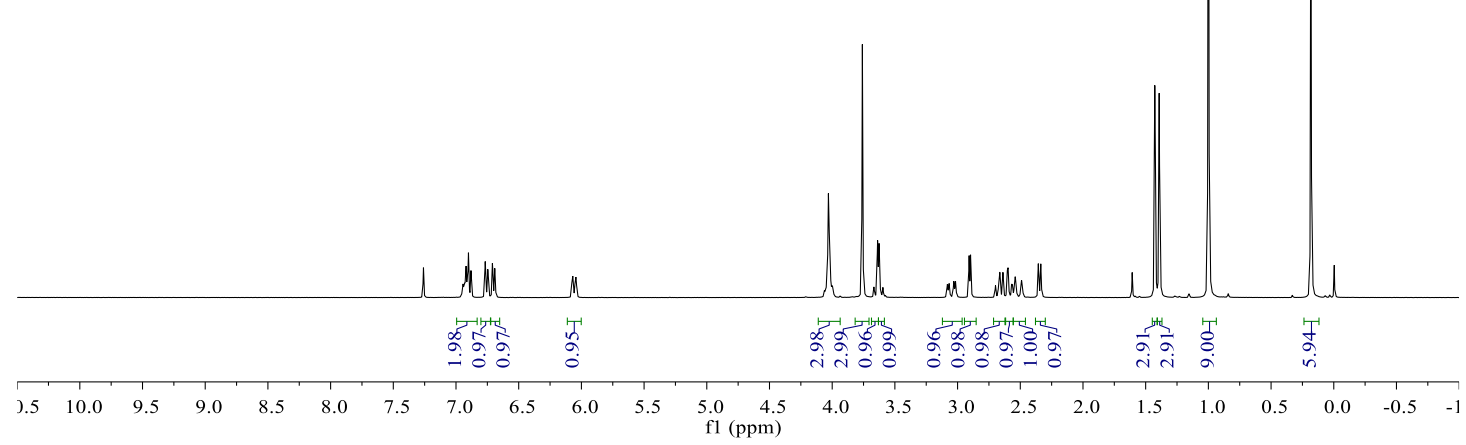

\begin{tabular}{|c|c|c|c|c|c|}
\hline \begin{tabular}{c}
\multirow{N}{N}{} \\
0
\end{tabular} & 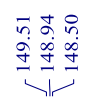 & 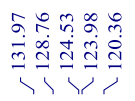 & $\begin{array}{c}0 \\
i \\
\infty \\
\infty \\
i\end{array}$ & 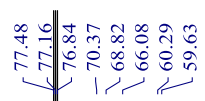 & 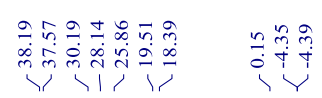 \\
\hline
\end{tabular}

${ }^{13} \mathrm{C}$ NMR spectrum $\left(100 \mathrm{MHz}, \mathrm{CDCl}_{3}\right)$

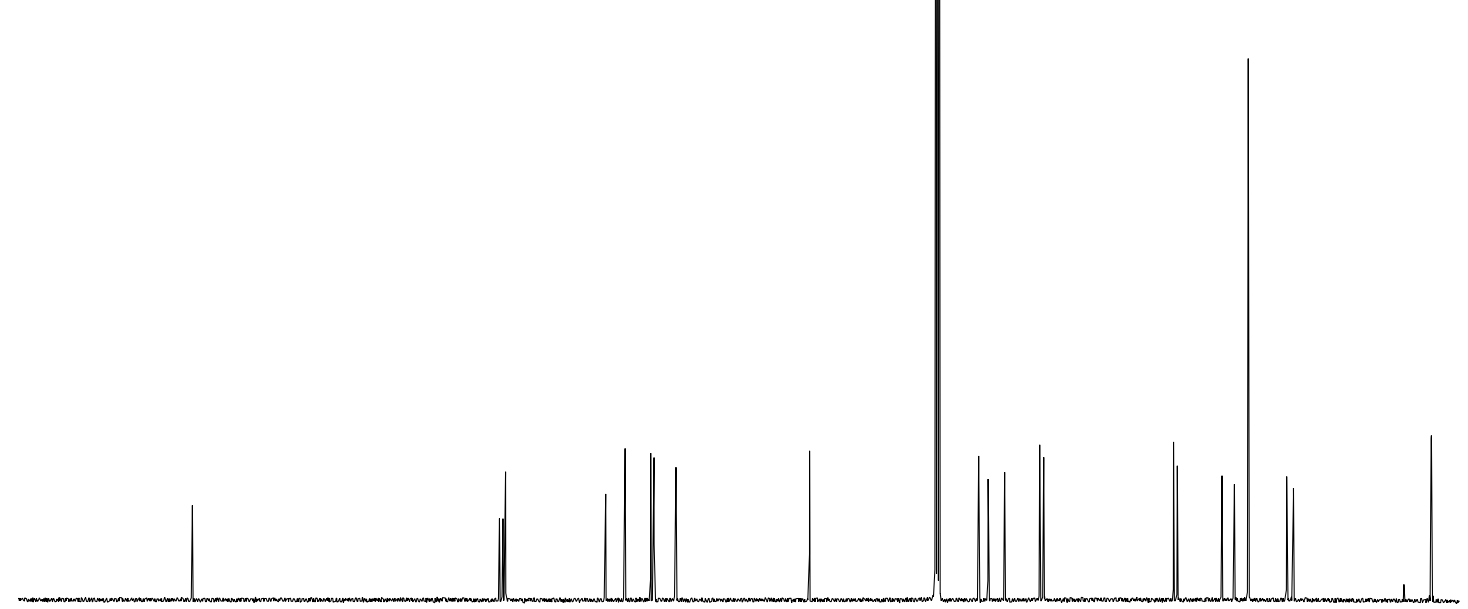

$\begin{array}{llllllllllllllllllllllll}220 & 210 & 200 & 190 & 180 & 170 & 160 & 150 & 140 & 130 & 120 & 110 & 100 & 90 & 80 & 70 & 60 & 50 & 40 & 30 & 20 & 10 & 0\end{array}$ 


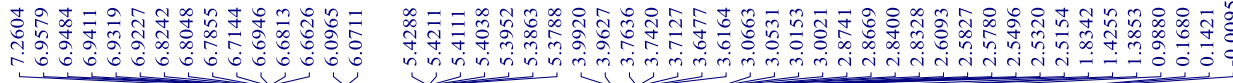

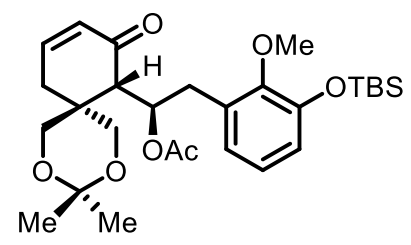

$(+)-20$

${ }^{1} \mathrm{H}$ NMR spectrum (400 MHz, $\mathrm{CDCl}_{3}$ )

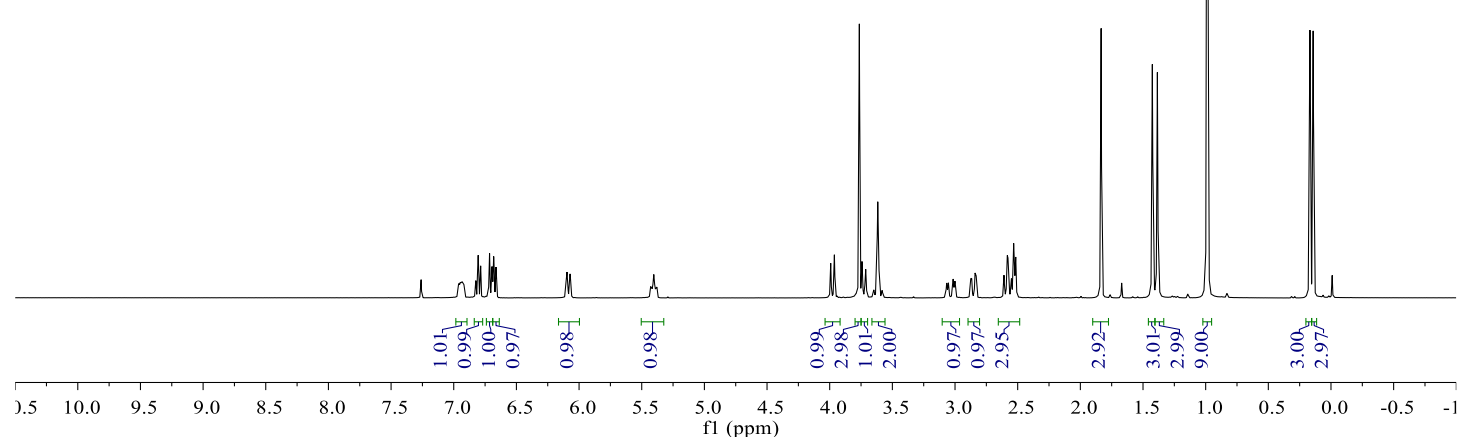

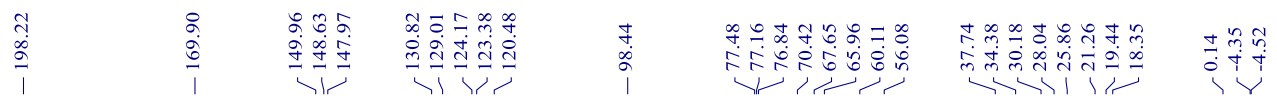

${ }^{13} \mathrm{C}$ NMR spectrum $\left(100 \mathrm{MHz}, \mathrm{CDCl}_{3}\right)$

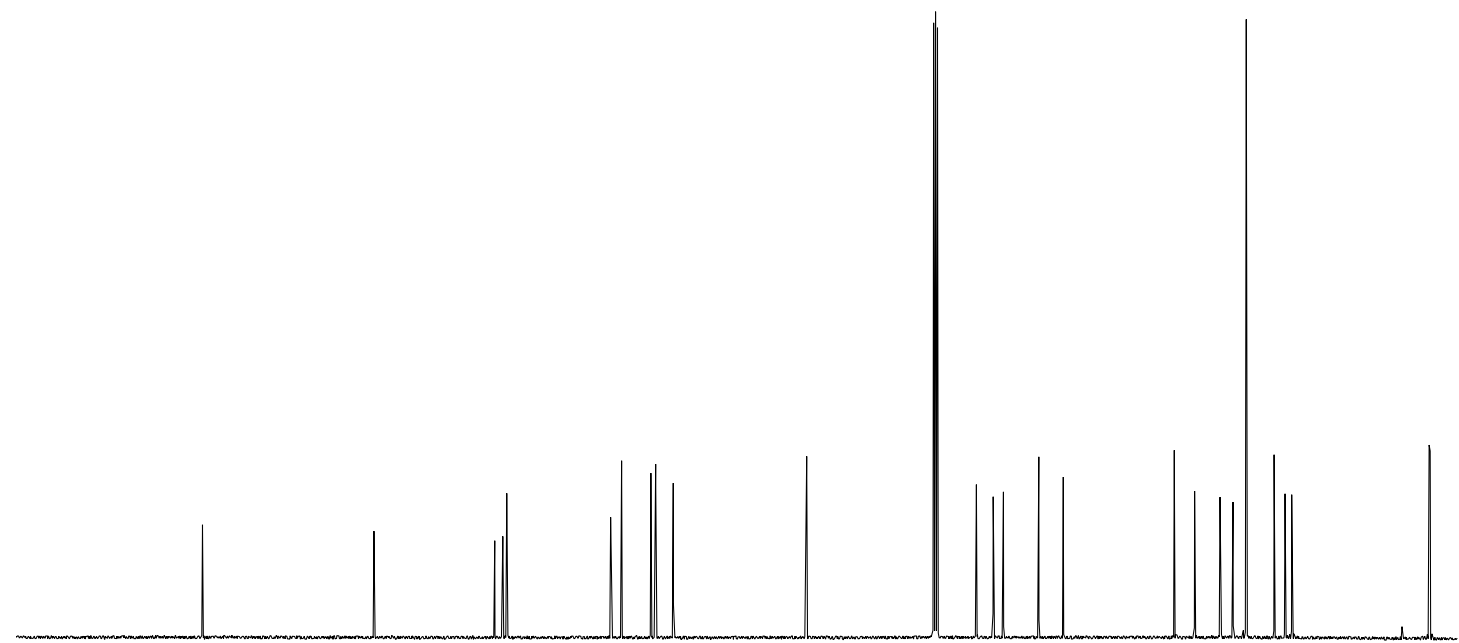

$\begin{array}{llllllllllllllllllllllll}220 & 210 & 200 & 190 & 180 & 170 & 160 & 150 & 140 & 130 & 120 & 110 & 100 & 90 & 80 & 70 & 60 & 50 & 40 & 30 & 20 & 10 & 0\end{array}$ 


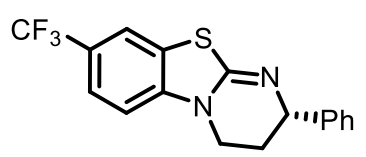

19

${ }^{1} \mathrm{H}$ NMR spectrum $\left(400 \mathrm{MHz}, \mathrm{CDCl}_{3}\right)$

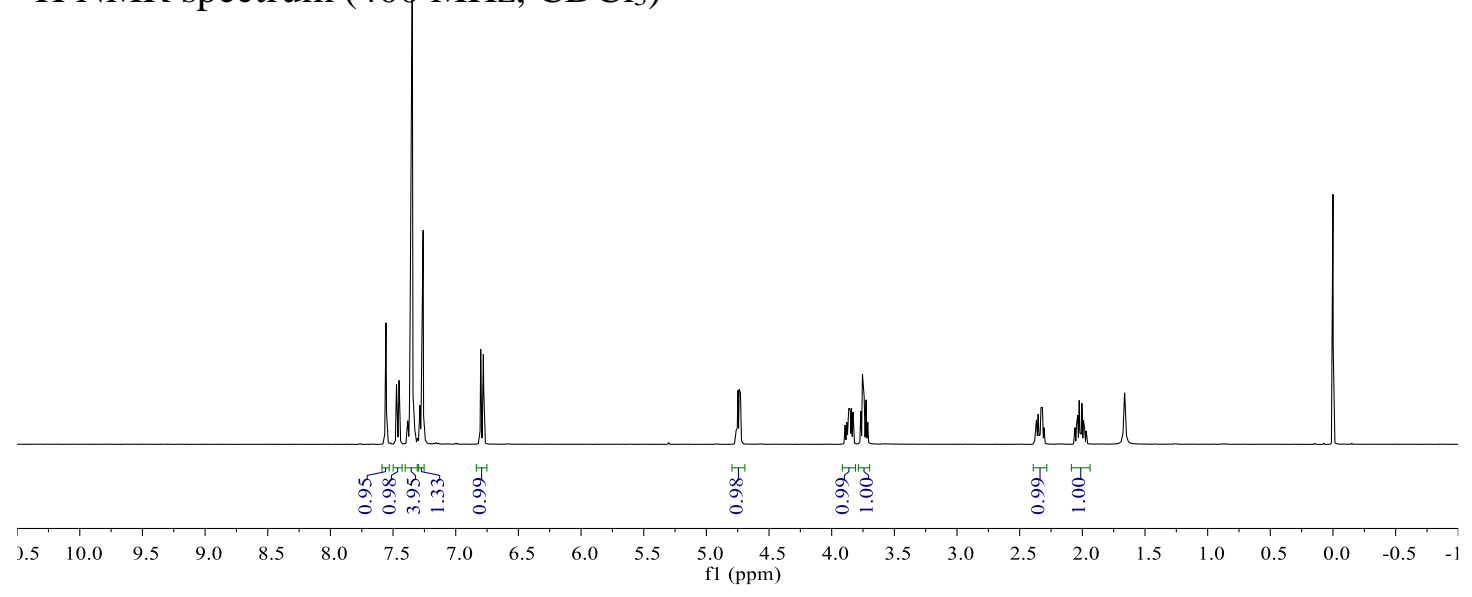

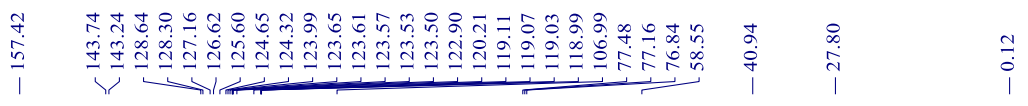

${ }^{13} \mathrm{C}$ NMR spectrum $\left(100 \mathrm{MHz}, \mathrm{CDCl}_{3}\right)$

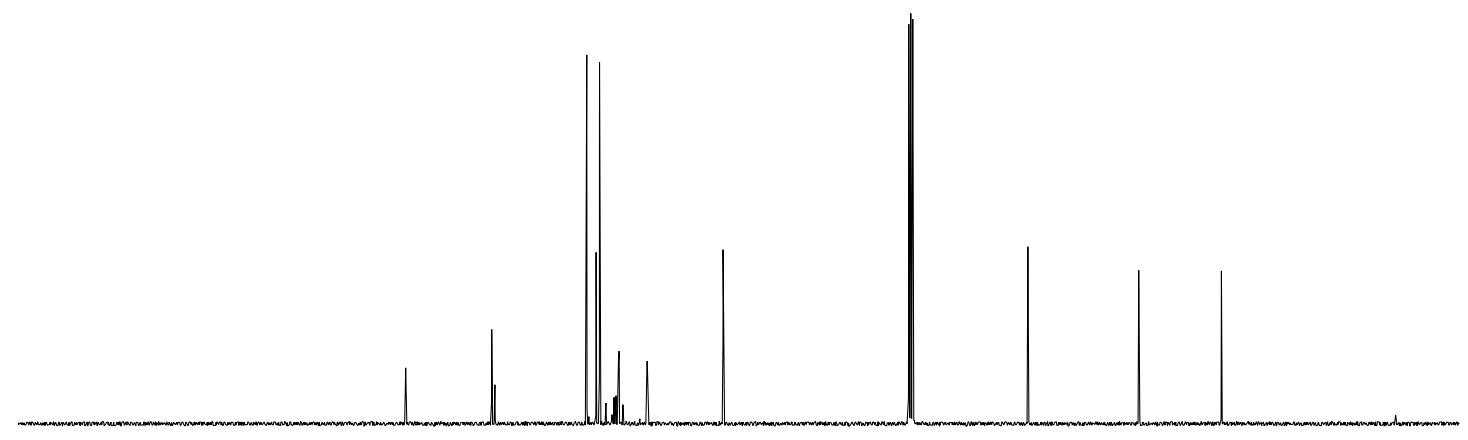

$\begin{array}{rlllllllllll}210 & 200 & 190 & 180 & 170 & 160 & 150 & 140 & 130 & 120 & 110 & 100 \\ \text { f1 (ppm) }\end{array}$ 


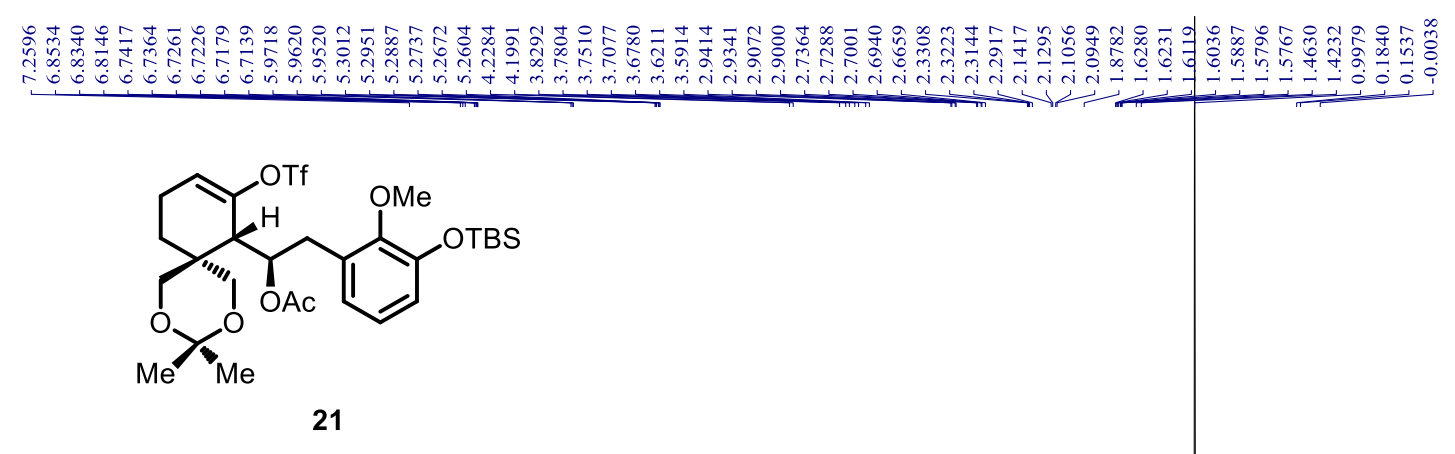

${ }^{1} \mathrm{H}$ NMR spectrum $\left(400 \mathrm{MHz}, \mathrm{CDCl}_{3}\right)$

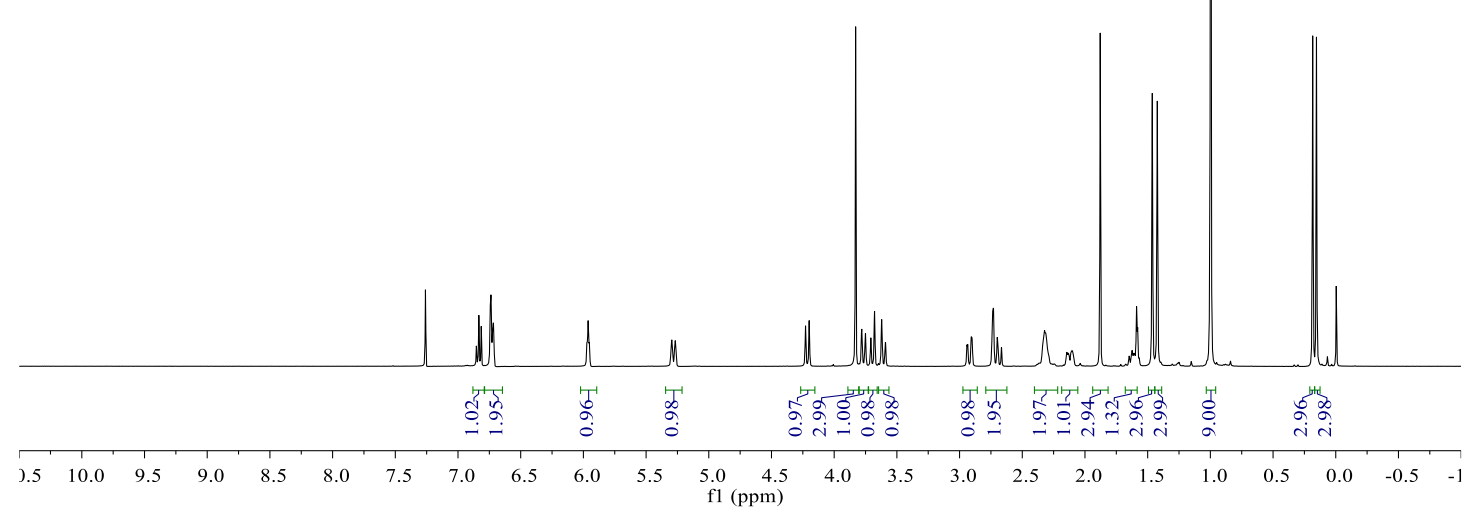

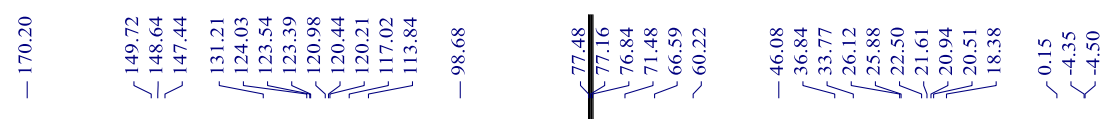

${ }^{13} \mathrm{C}$ NMR spectrum $\left(100 \mathrm{MHz}, \mathrm{CDCl}_{3}\right)$

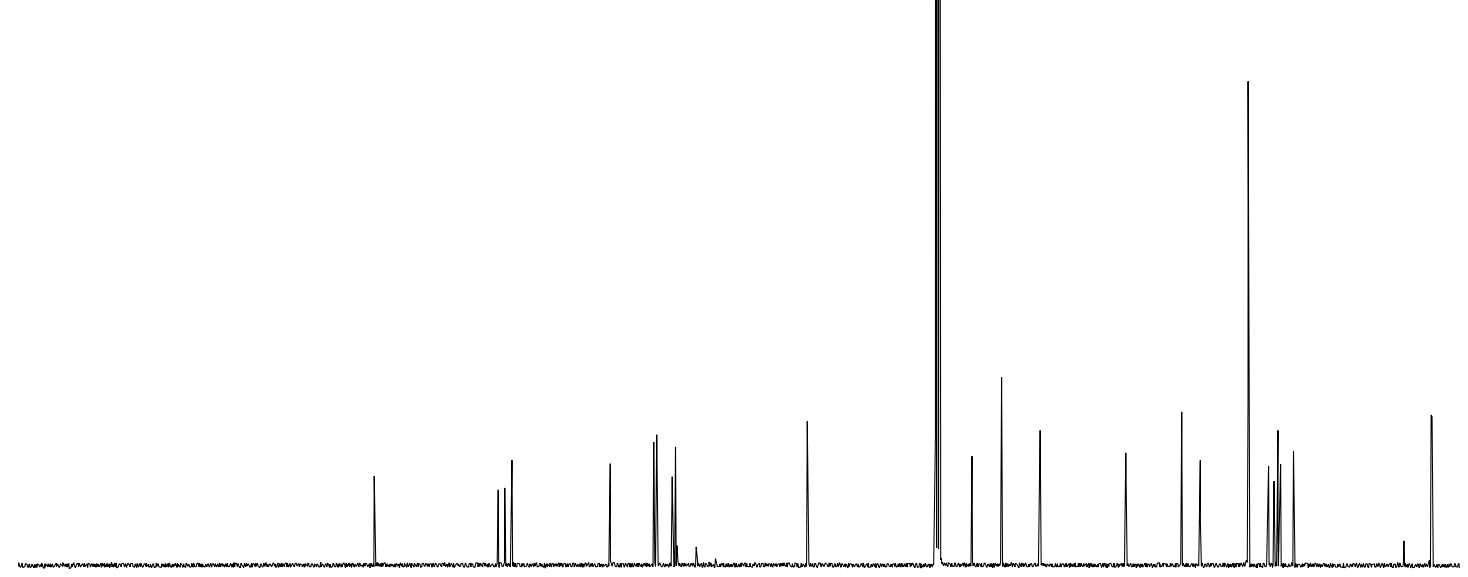

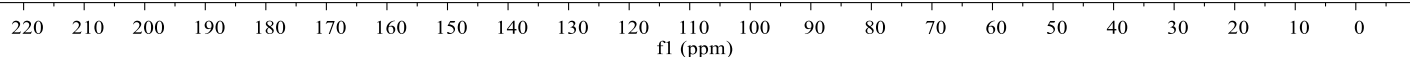


<smiles>C=CC(=O)C1=CCCC2(COC(C)(C)OC2)C1C(Cc1cccc(O)c1OC)OC</smiles>

${ }^{1} \mathrm{H}$ NMR spectrum (400 MHz, $\left.\mathrm{CDCl}_{3}\right)$

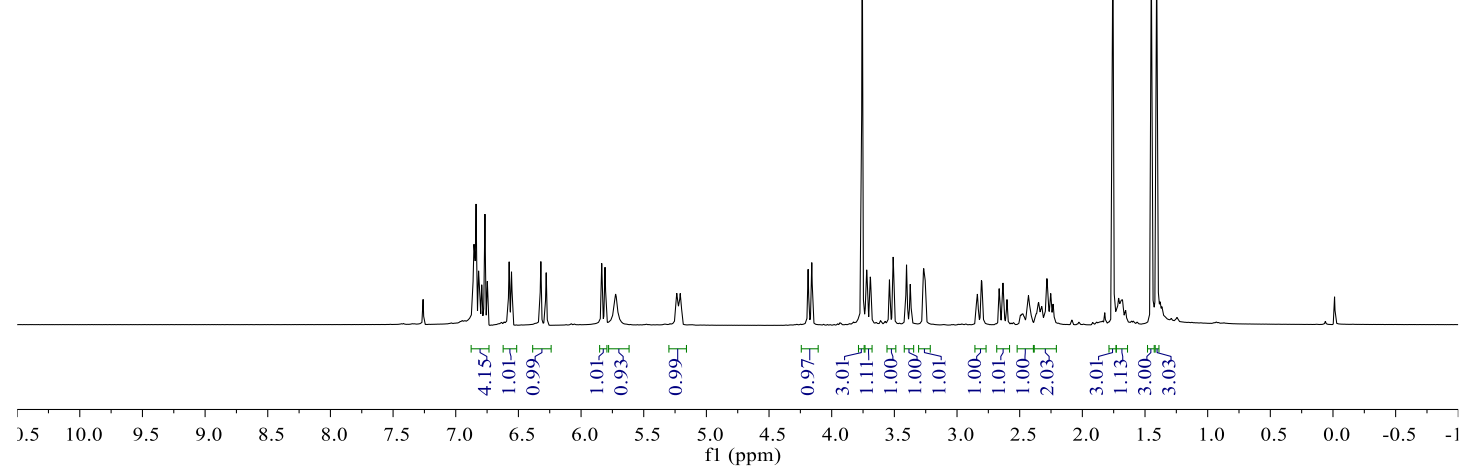

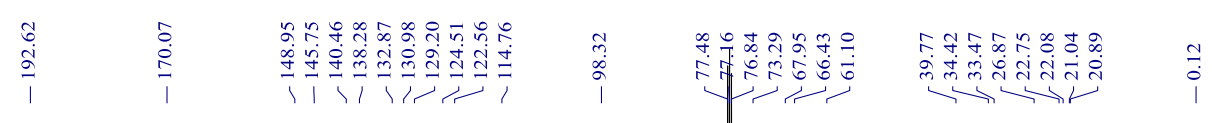

${ }^{13} \mathrm{C}$ NMR spectrum $\left(100 \mathrm{MHz}, \mathrm{CDCl}_{3}\right)$

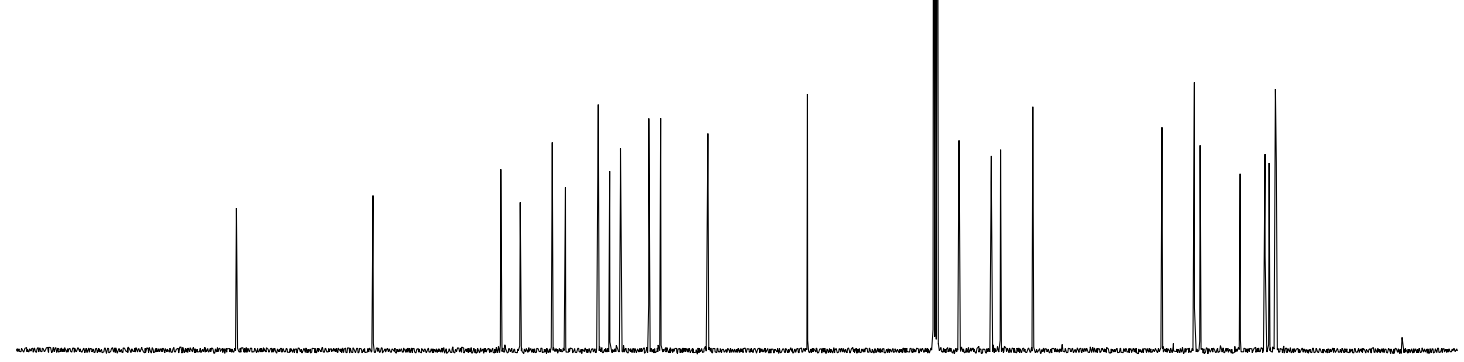

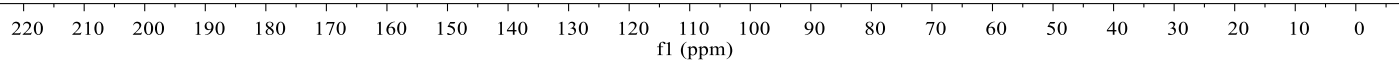




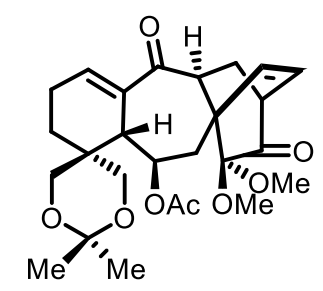

22

${ }^{1} \mathrm{H}$ NMR spectrum (400 MHz, $\mathrm{CDCl}_{3}$ )

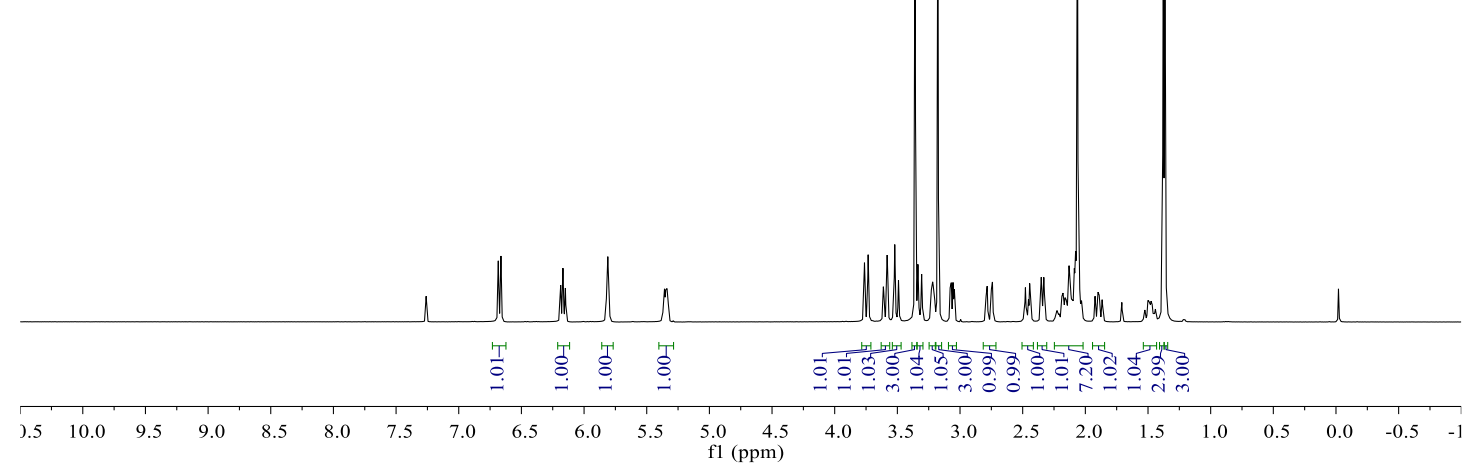

${ }^{13} \mathrm{C}$ NMR spectrum $\left(100 \mathrm{MHz}, \mathrm{CDCl}_{3}\right)$ लेखें

\section{C}

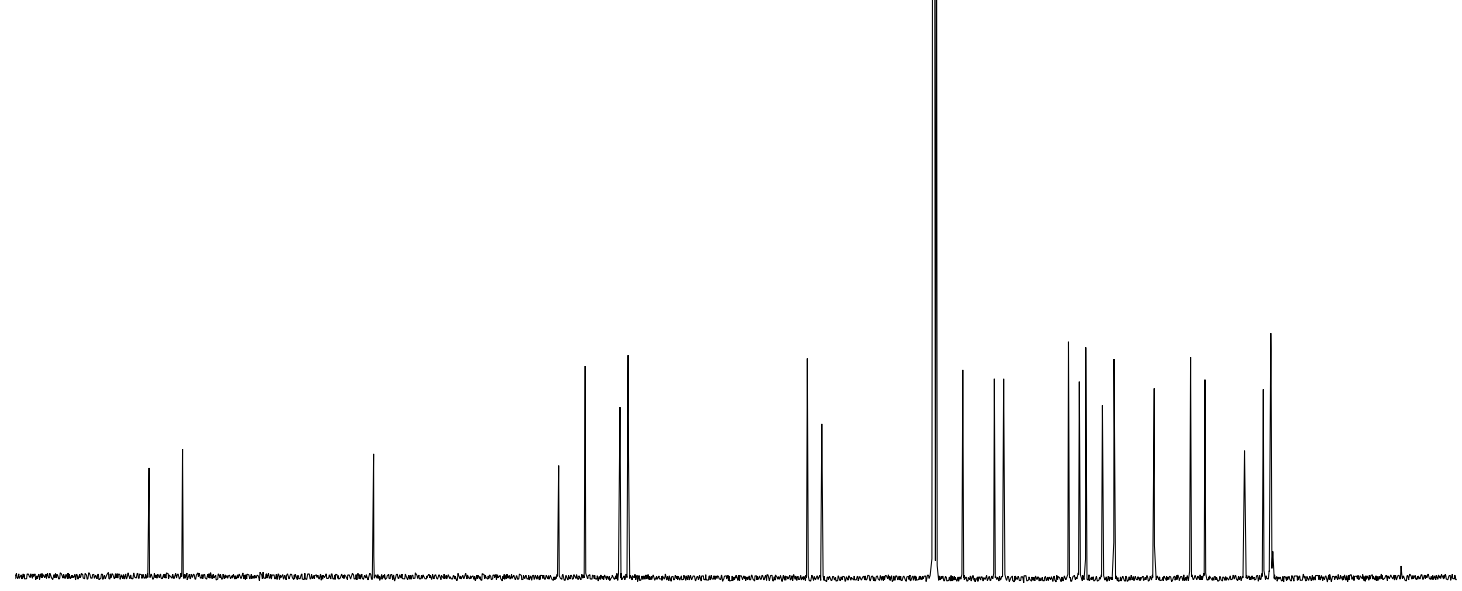

$\begin{array}{llllllllllllllllllllllllll}220 & 210 & 200 & 190 & 180 & 170 & 160 & 150 & 140 & 130 & 120 & 110 & 100 & 90 & 80 & 70 & 60 & 50 & 40 & 30 & 20 & 10 & 0\end{array}$ 


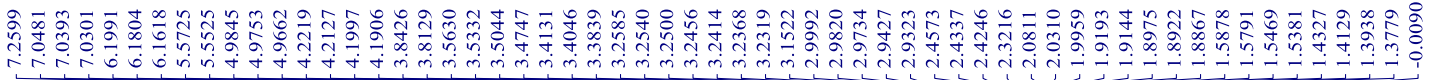

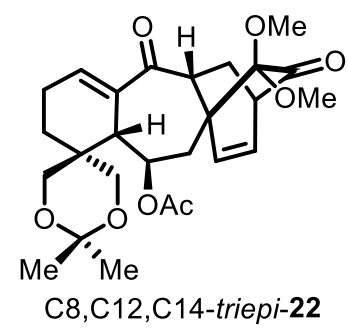

${ }^{1} \mathrm{H}$ NMR spectrum (400 MHz, $\mathrm{CDCl}_{3}$ )

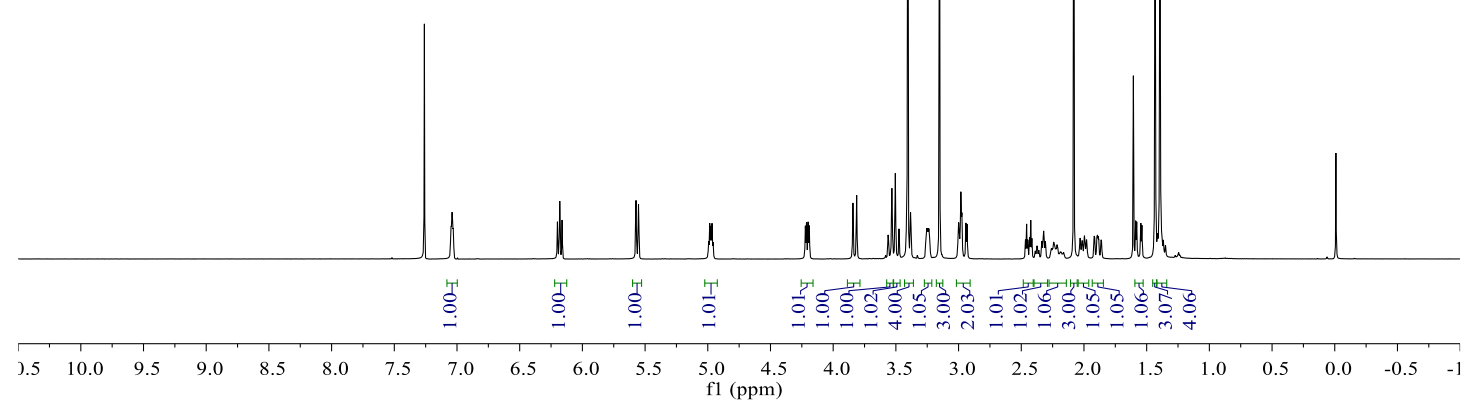

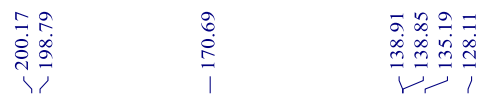

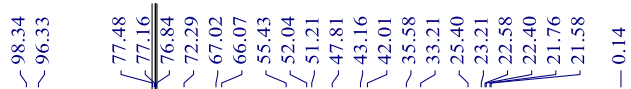

${ }^{13} \mathrm{C}$ NMR spectrum $\left(100 \mathrm{MHz}, \mathrm{CDCl}_{3}\right)$

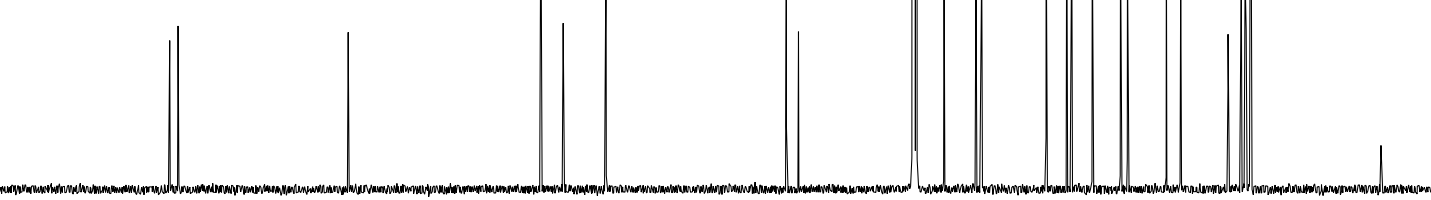

$\begin{array}{lllllllllllllllllllllll}220 & 210 & 200 & 190 & 180 & 170 & 160 & 150 & 140 & 130 & 120 & 110 & 100 & 90 & 80 & 70 & 60 & 50 & 40 & 30 & 20 & 10 & 0\end{array}$ 

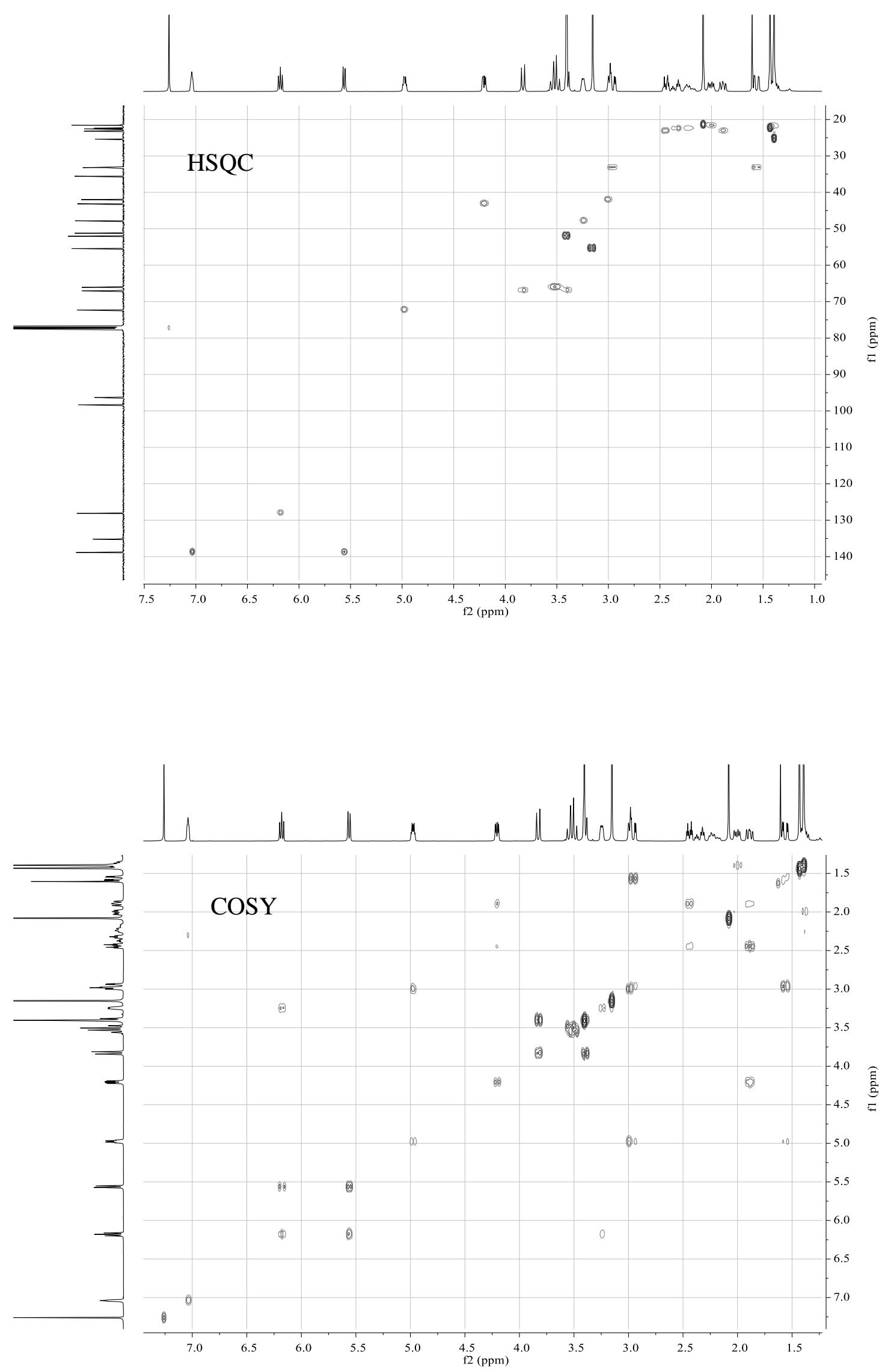

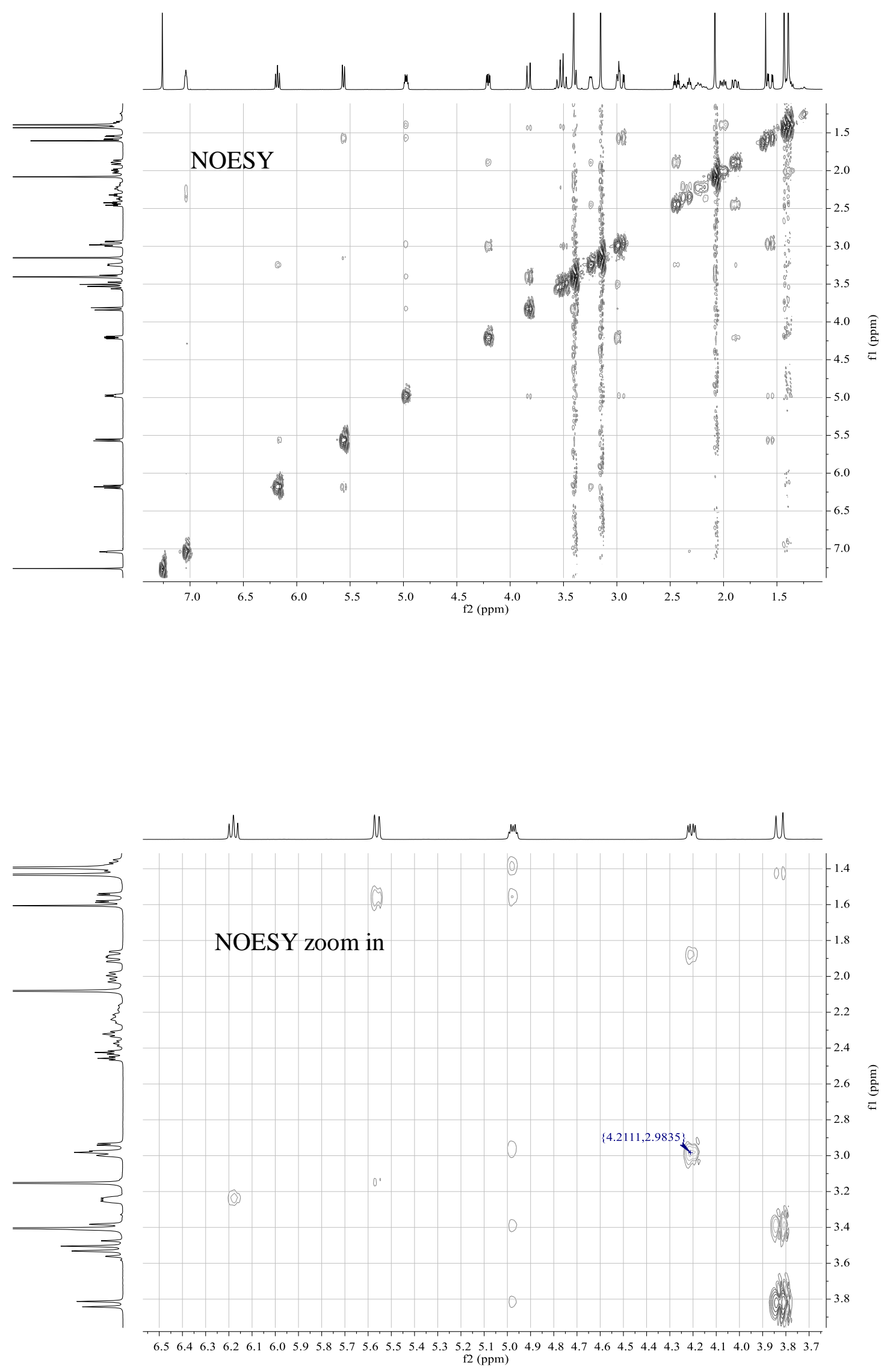


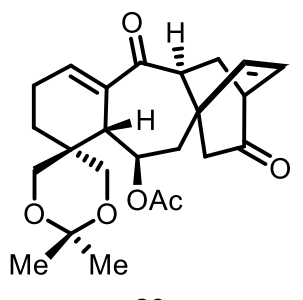

23

${ }^{1} \mathrm{H}$ NMR spectrum $\left(400 \mathrm{MHz}, \mathrm{CDCl}_{3}\right)$

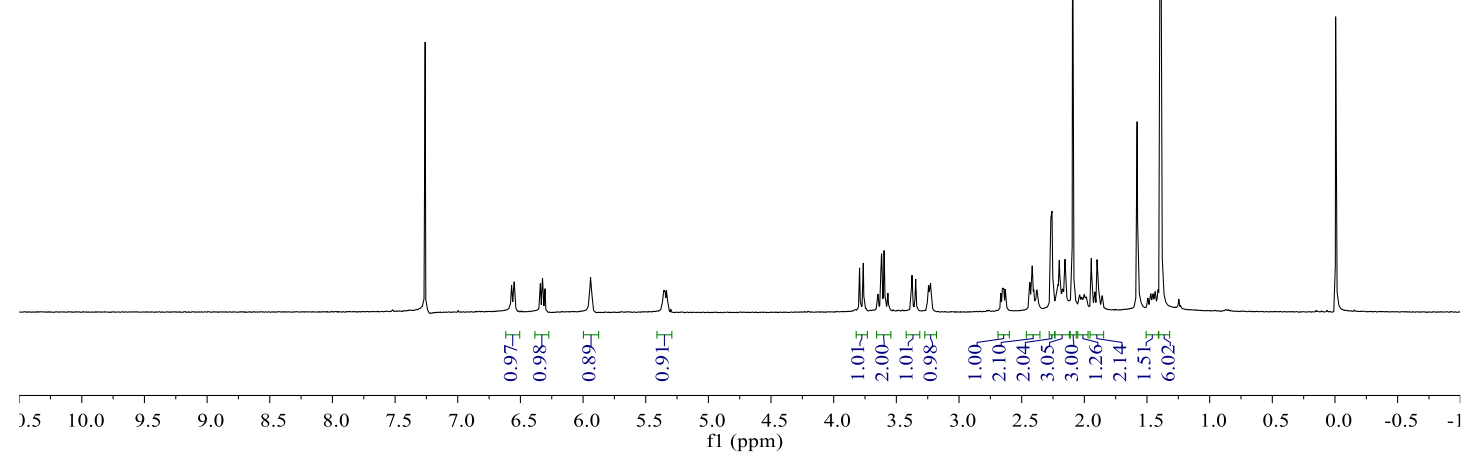

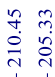
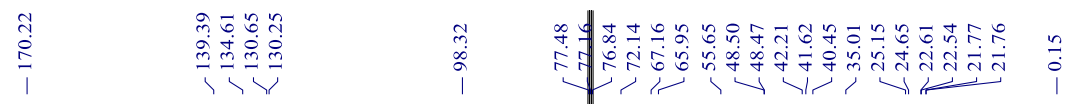

${ }^{13} \mathrm{C}$ NMR spectrum $\left(100 \mathrm{MHz}, \mathrm{CDCl}_{3}\right)$

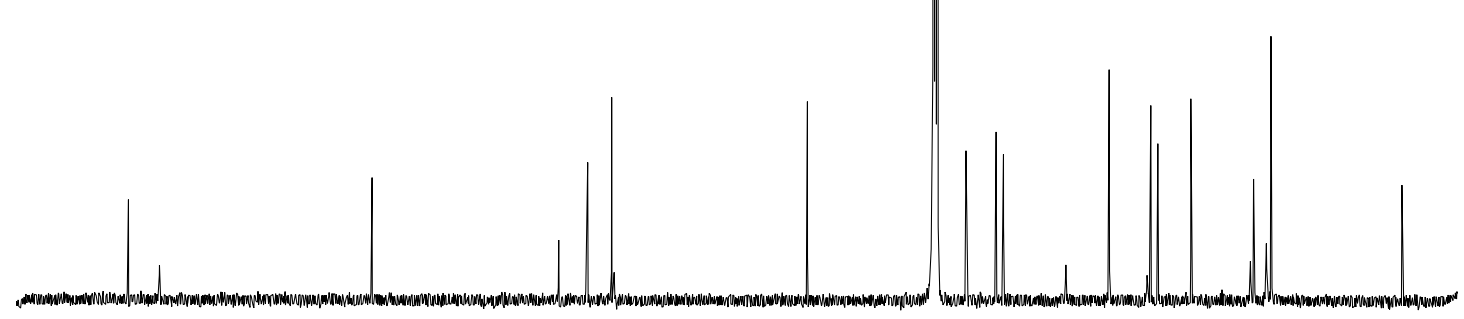

$\begin{array}{lllllllllllllllllllllll}220 & 210 & 200 & 190 & 180 & 170 & 160 & 150 & 140 & 130 & 120 & 110 & 100 & 90 & 80 & 70 & 60 & 50 & 40 & 30 & 20 & 10 & 0\end{array}$ 


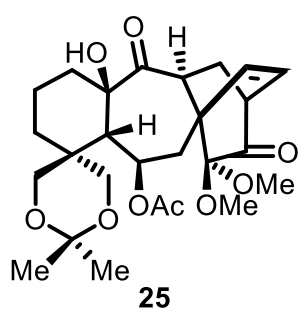

${ }^{1} \mathrm{H}$ NMR spectrum $\left(400 \mathrm{MHz}, \mathrm{CDCl}_{3}\right)$

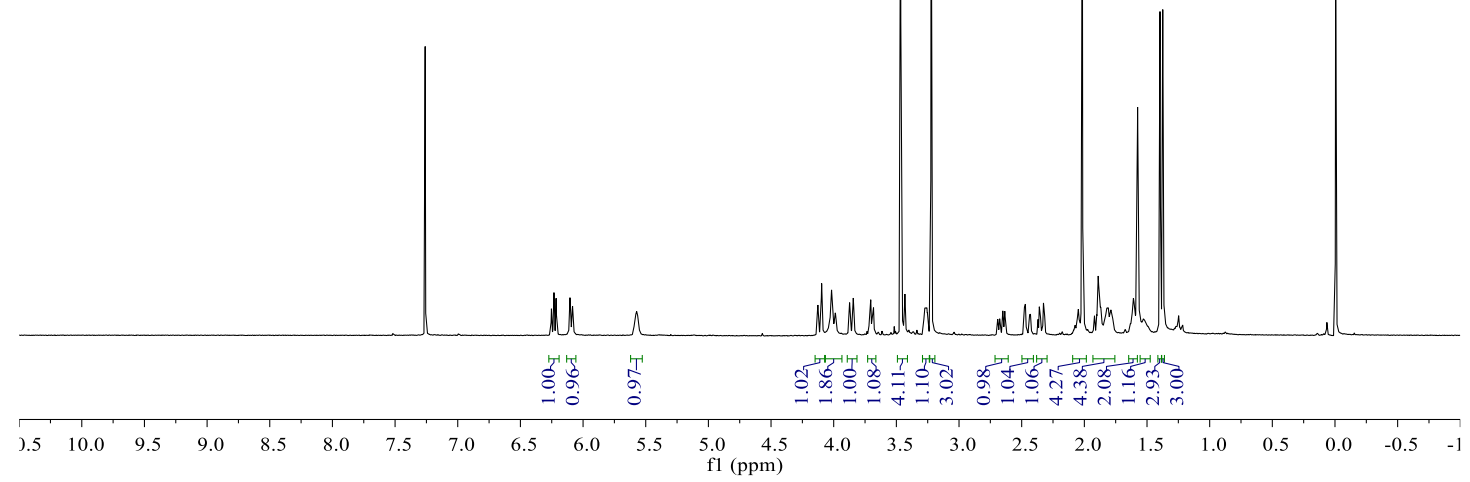

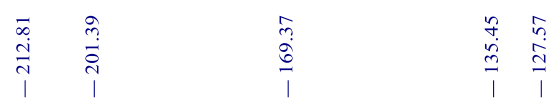

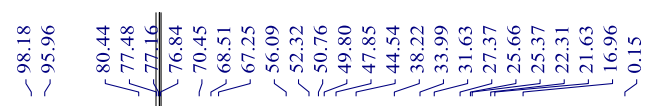

${ }^{13} \mathrm{C}$ NMR spectrum $\left(100 \mathrm{MHz}, \mathrm{CDCl}_{3}\right)$

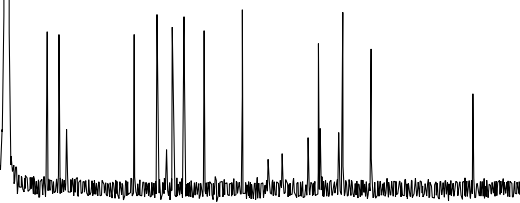

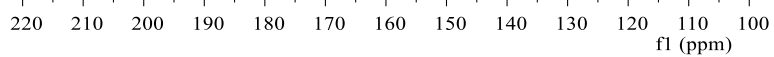



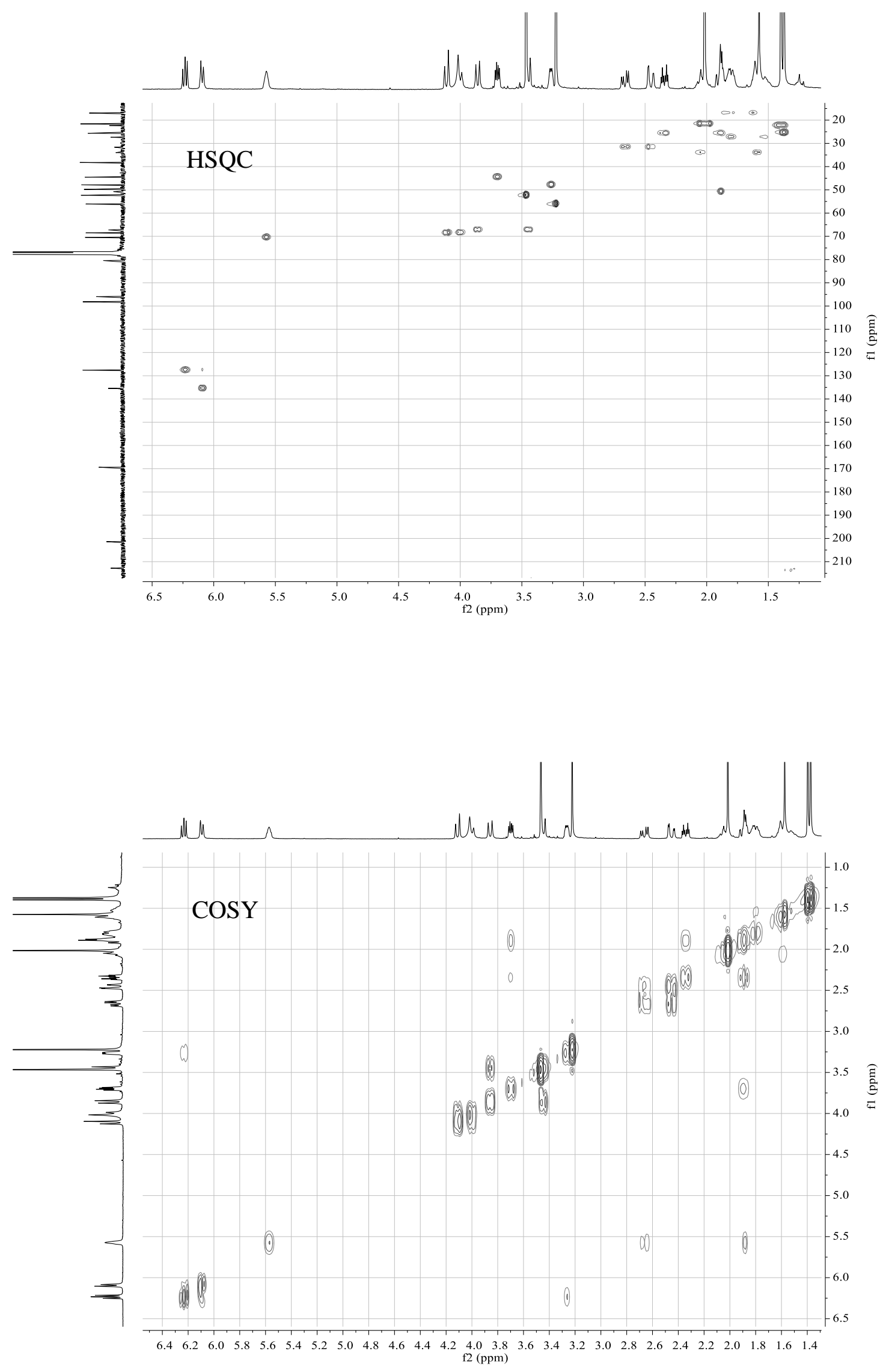

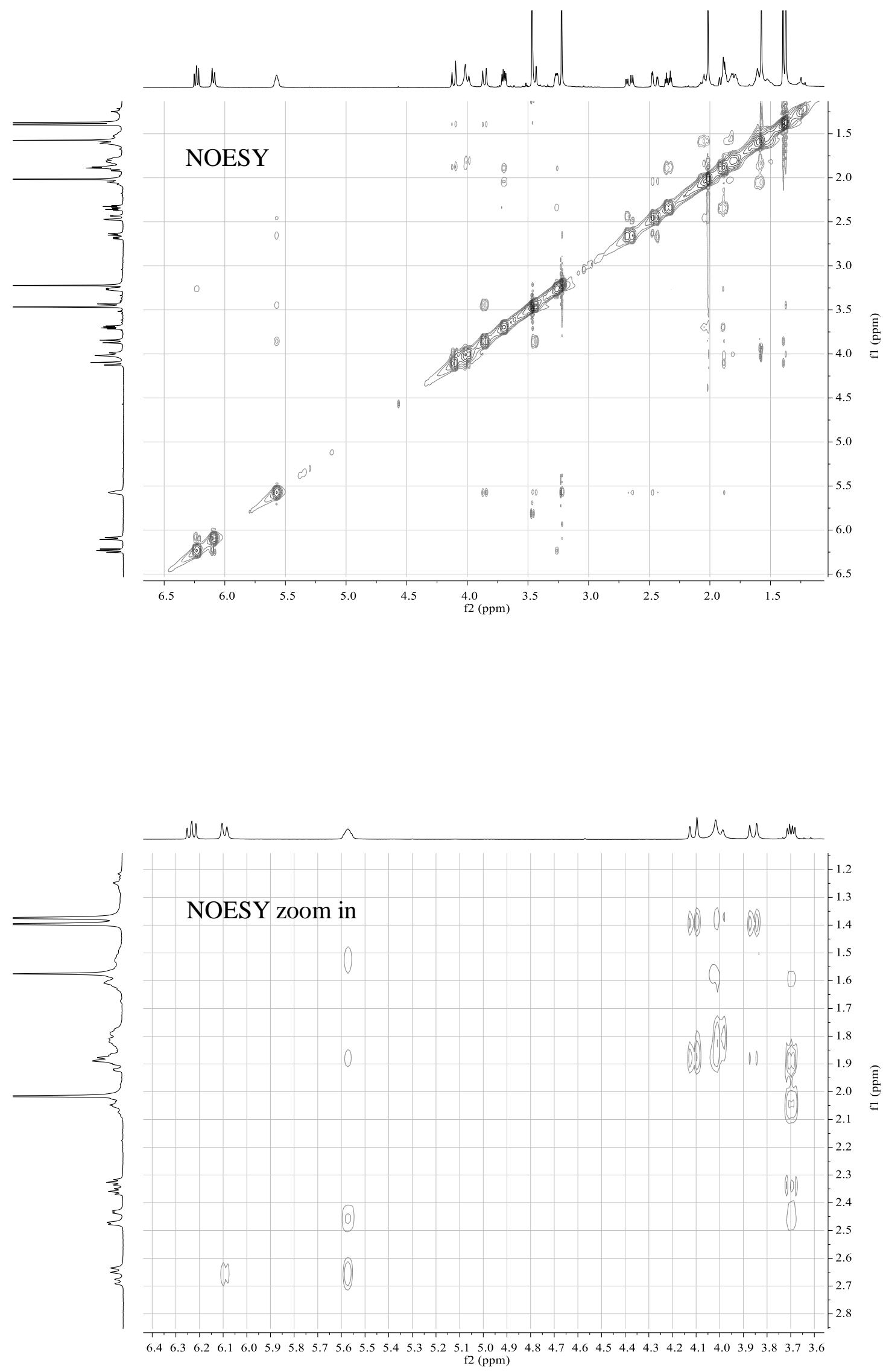


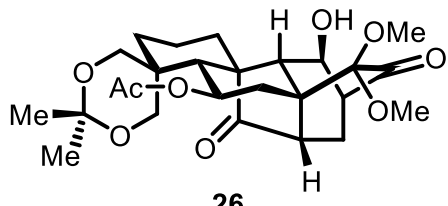

26

${ }^{1} \mathrm{H}$ NMR spectrum $\left(400 \mathrm{MHz}, \mathrm{CDCl}_{3}\right)$

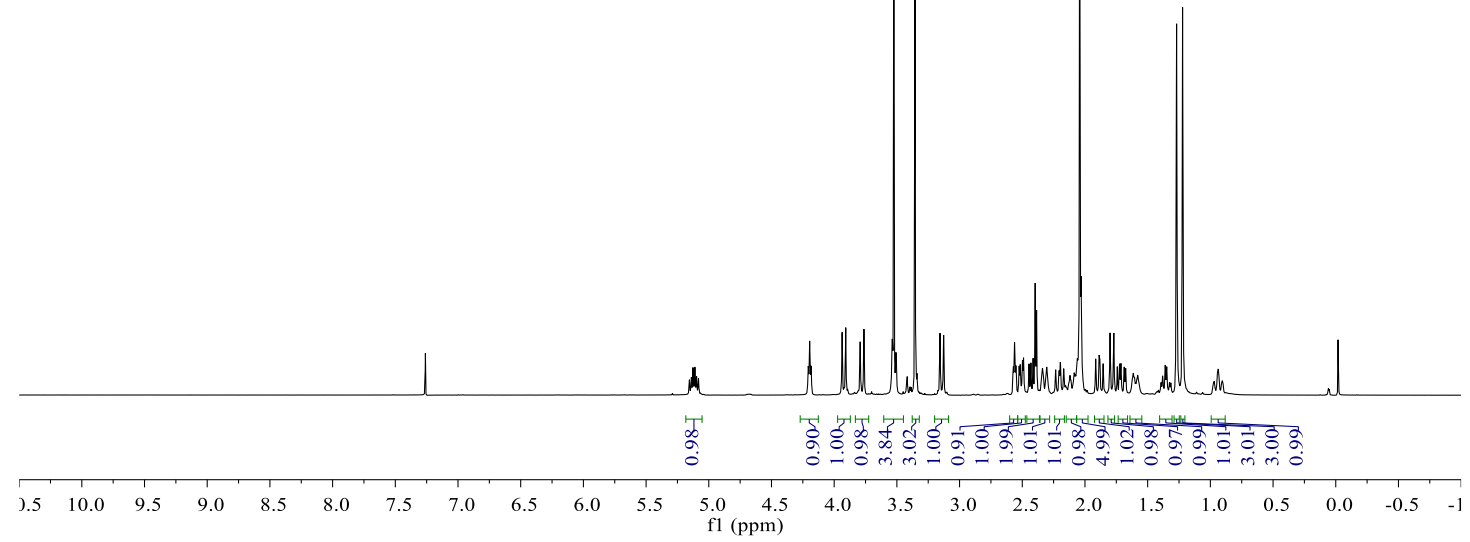

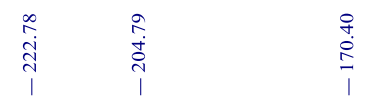

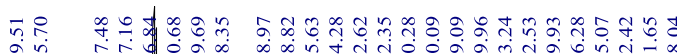

${ }^{13} \mathrm{C}$ NMR spectrum $\left(100 \mathrm{MHz}, \mathrm{CDCl}_{3}\right)$

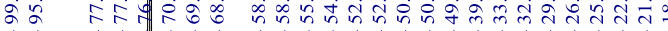

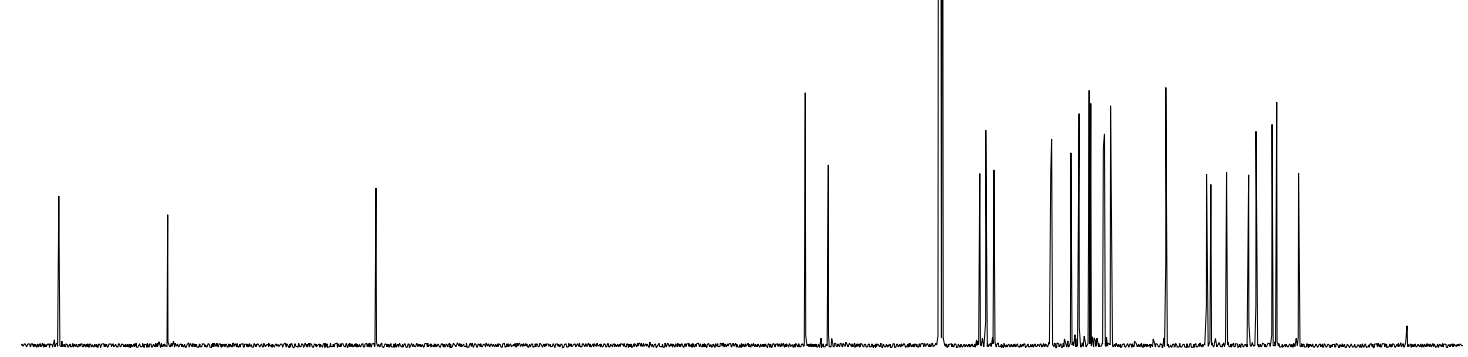

$\begin{array}{llllllllllllllllllllllllllll}220 & 210 & 200 & 190 & 180 & 170 & 160 & 150 & 140 & 130 & 120 & 110 & 100 & 90 & 80 & 70 & 60 & 50 & 40 & 30 & 20 & 10 & 0\end{array}$ 


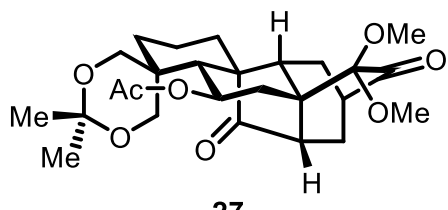

27

${ }^{1} \mathrm{H}$ NMR spectrum $\left(400 \mathrm{MHz}, \mathrm{CDCl}_{3}\right)$

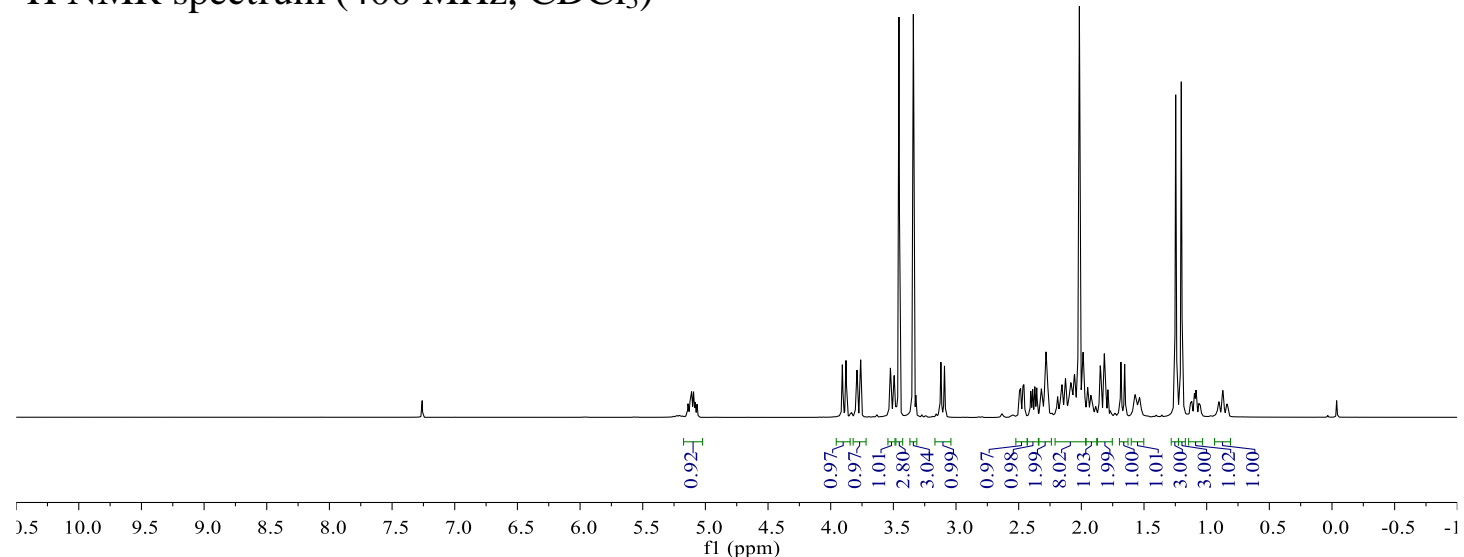

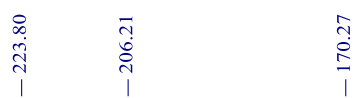

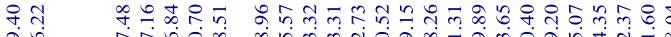

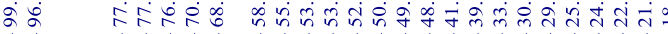

${ }^{13} \mathrm{C}$ NMR spectrum $\left(100 \mathrm{MHz}, \mathrm{CDCl}_{3}\right)$

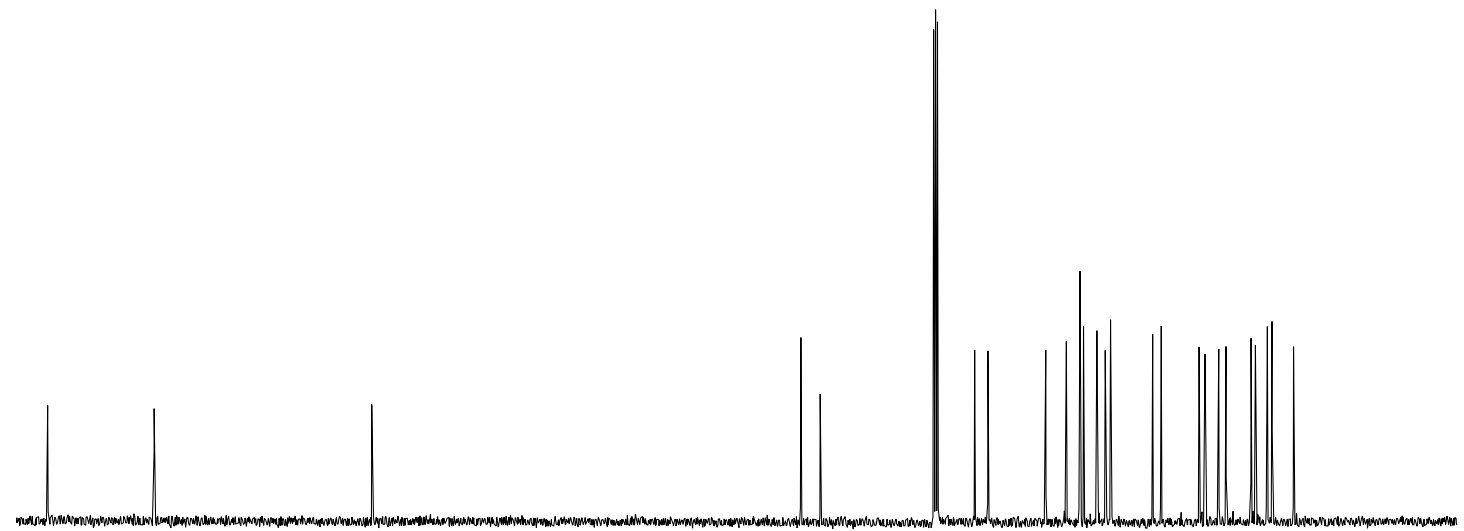

$\begin{array}{lllllllllllllllllllllll}220 & 210 & 200 & 190 & 180 & 170 & 160 & 150 & 140 & 130 & 120 & 110 & 100 & 90 & 80 & 70 & 60 & 50 & 40 & 30 & 20 & 10 & 0\end{array}$ 


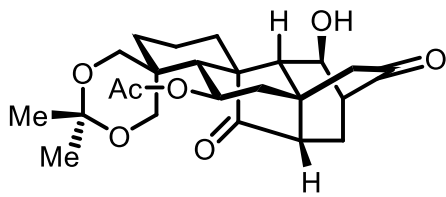

S-4

${ }^{1} \mathrm{H}$ NMR spectrum $\left(400 \mathrm{MHz}, \mathrm{CDCl}_{3}\right)$

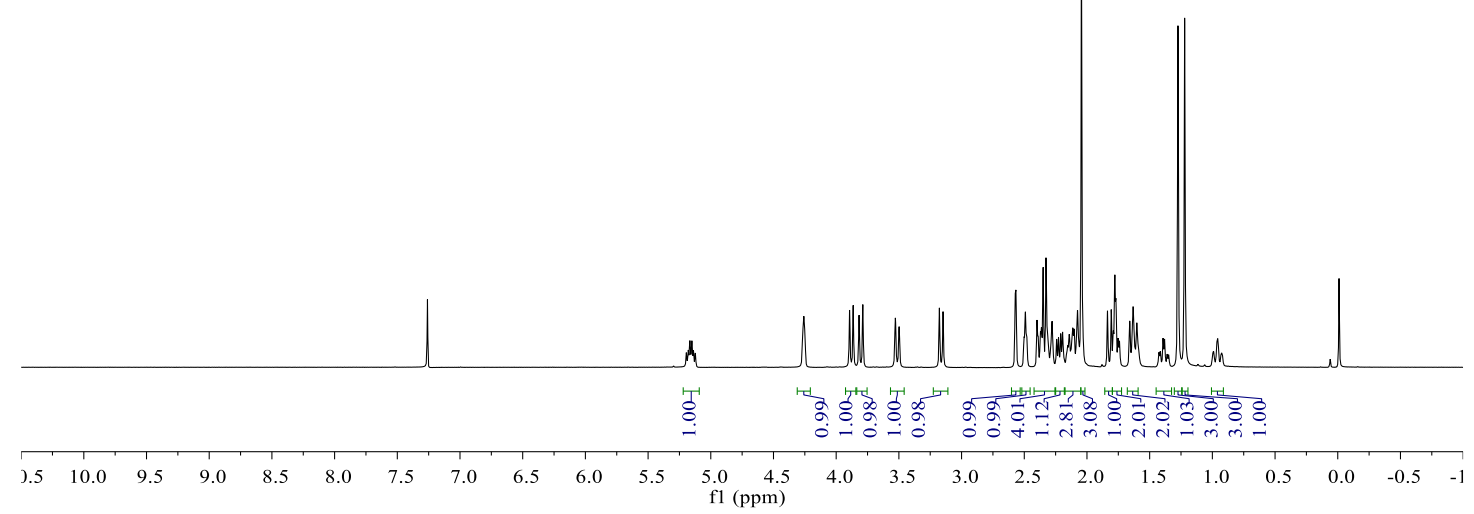

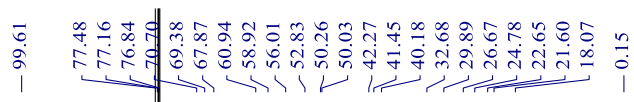

${ }^{13} \mathrm{C}$ NMR spectrum $\left(100 \mathrm{MHz}, \mathrm{CDCl}_{3}\right)$

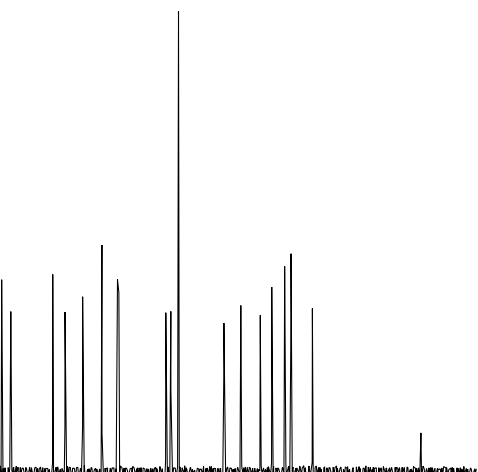

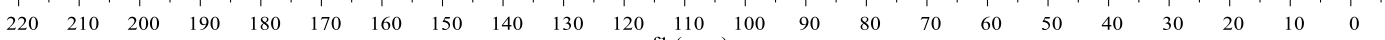




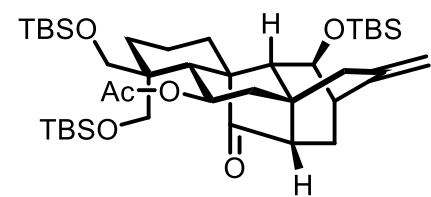

S-5

${ }^{1} \mathrm{H}$ NMR spectrum $\left(400 \mathrm{MHz}, \mathrm{CDCl}_{3}\right)$

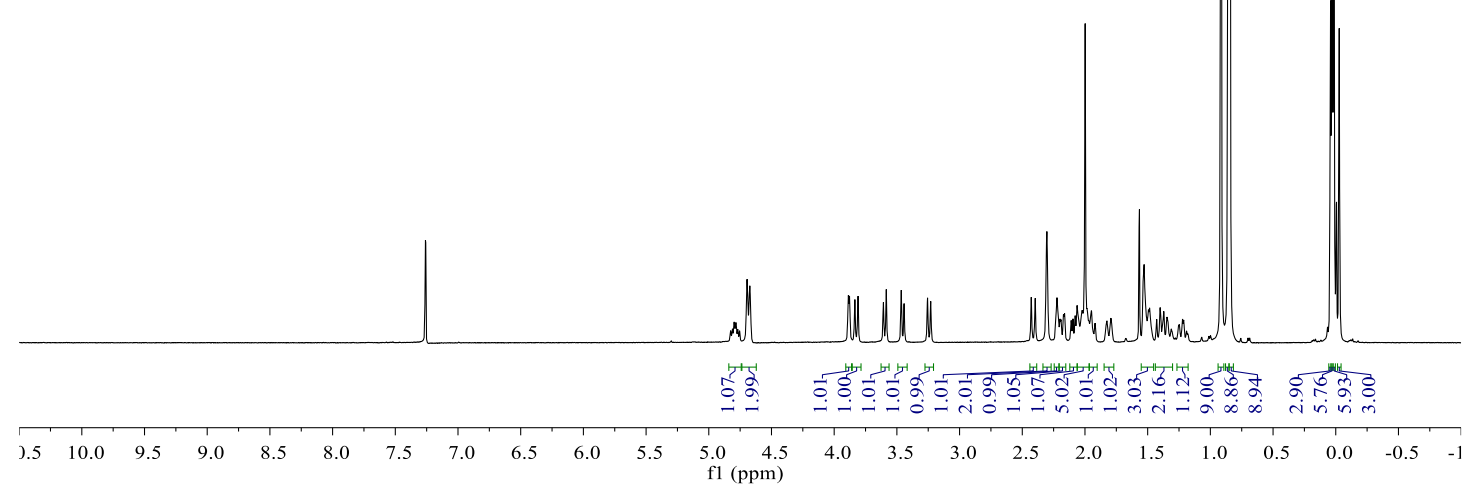

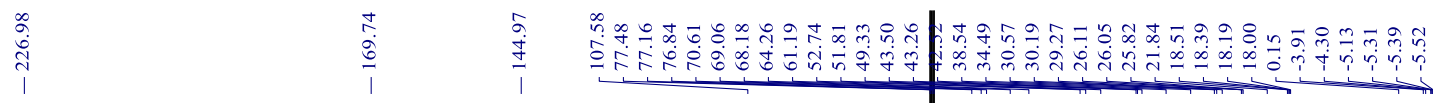

${ }^{13} \mathrm{C}$ NMR spectrum $\left(100 \mathrm{MHz}, \mathrm{CDCl}_{3}\right)$

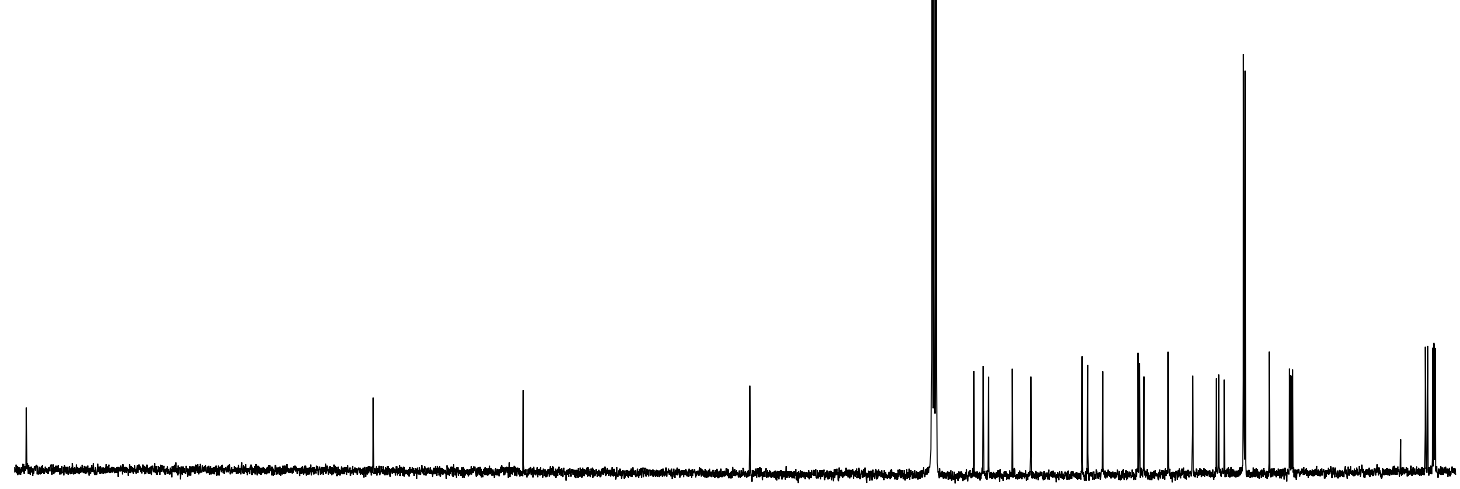

$\begin{array}{llllllllllllllllllllllll}220 & 210 & 200 & 190 & 180 & 170 & 160 & 150 & 140 & 130 & 120 & 110 & 100 & 90 & 80 & 70 & 60 & 50 & 40 & 30 & 20 & 10 & 0\end{array}$ 


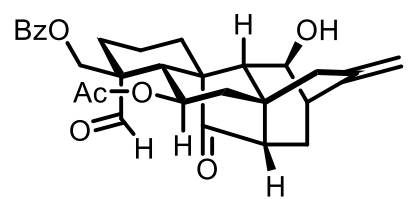

12

${ }^{1} \mathrm{H}$ NMR spectrum $\left(400 \mathrm{MHz}, \mathrm{CDCl}_{3}\right)$

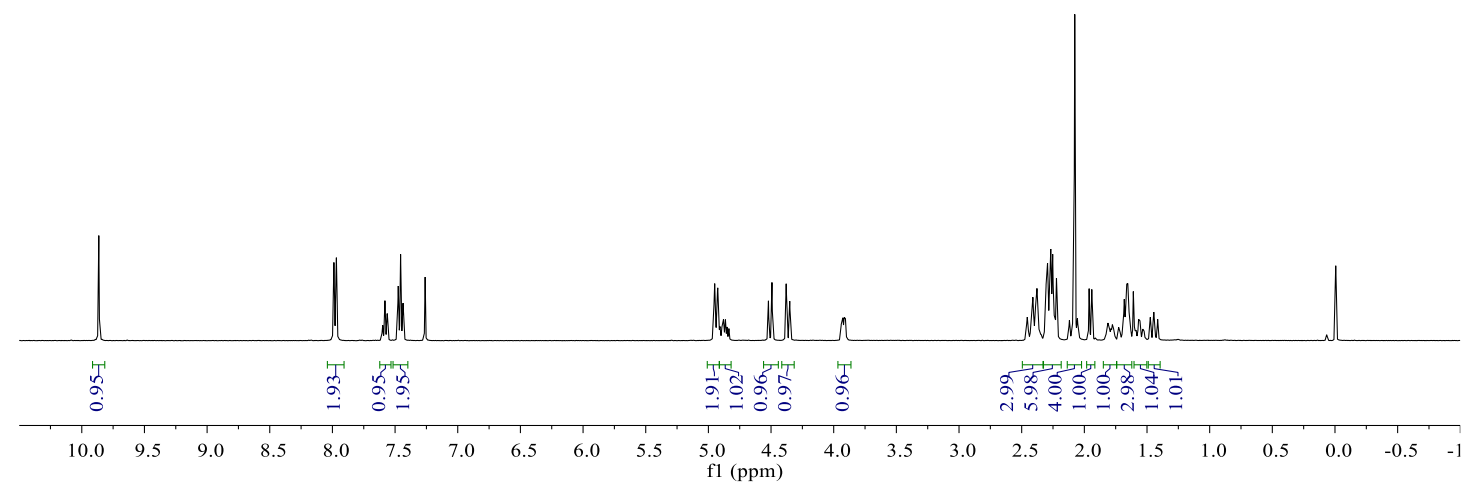

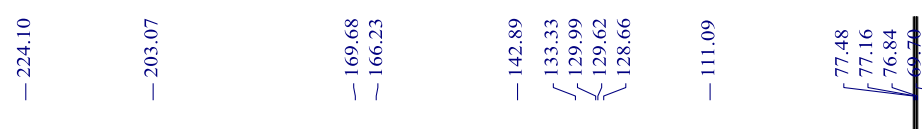

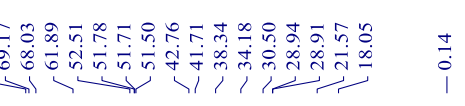

${ }^{13} \mathrm{C}$ NMR spectrum $\left(100 \mathrm{MHz}, \mathrm{CDCl}_{3}\right)$

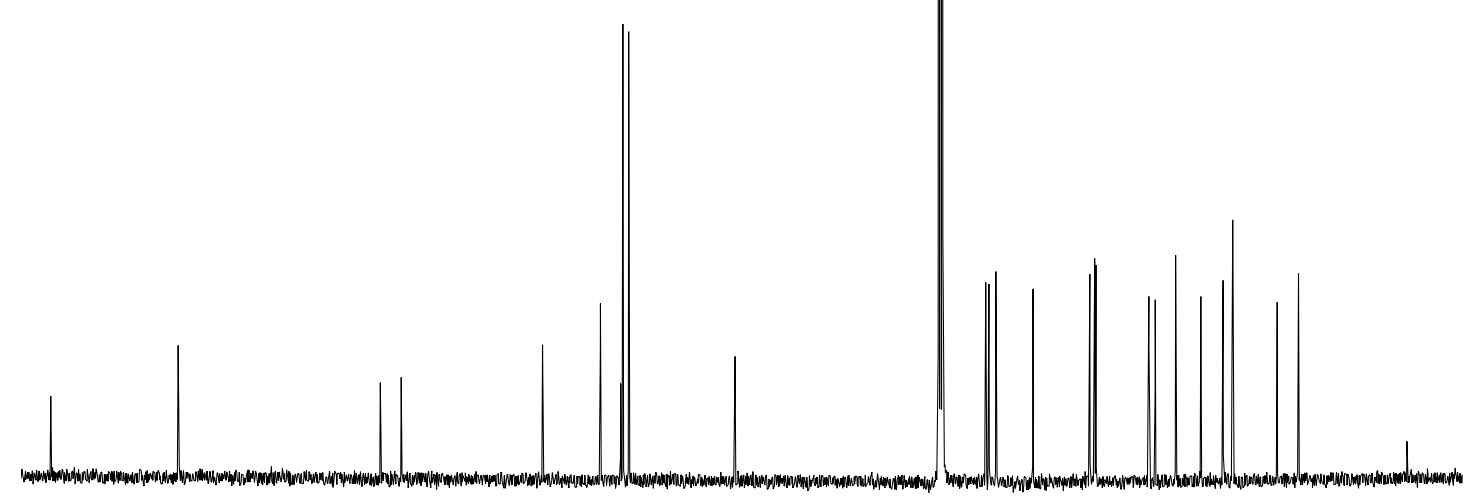

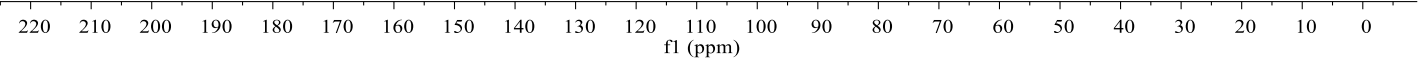



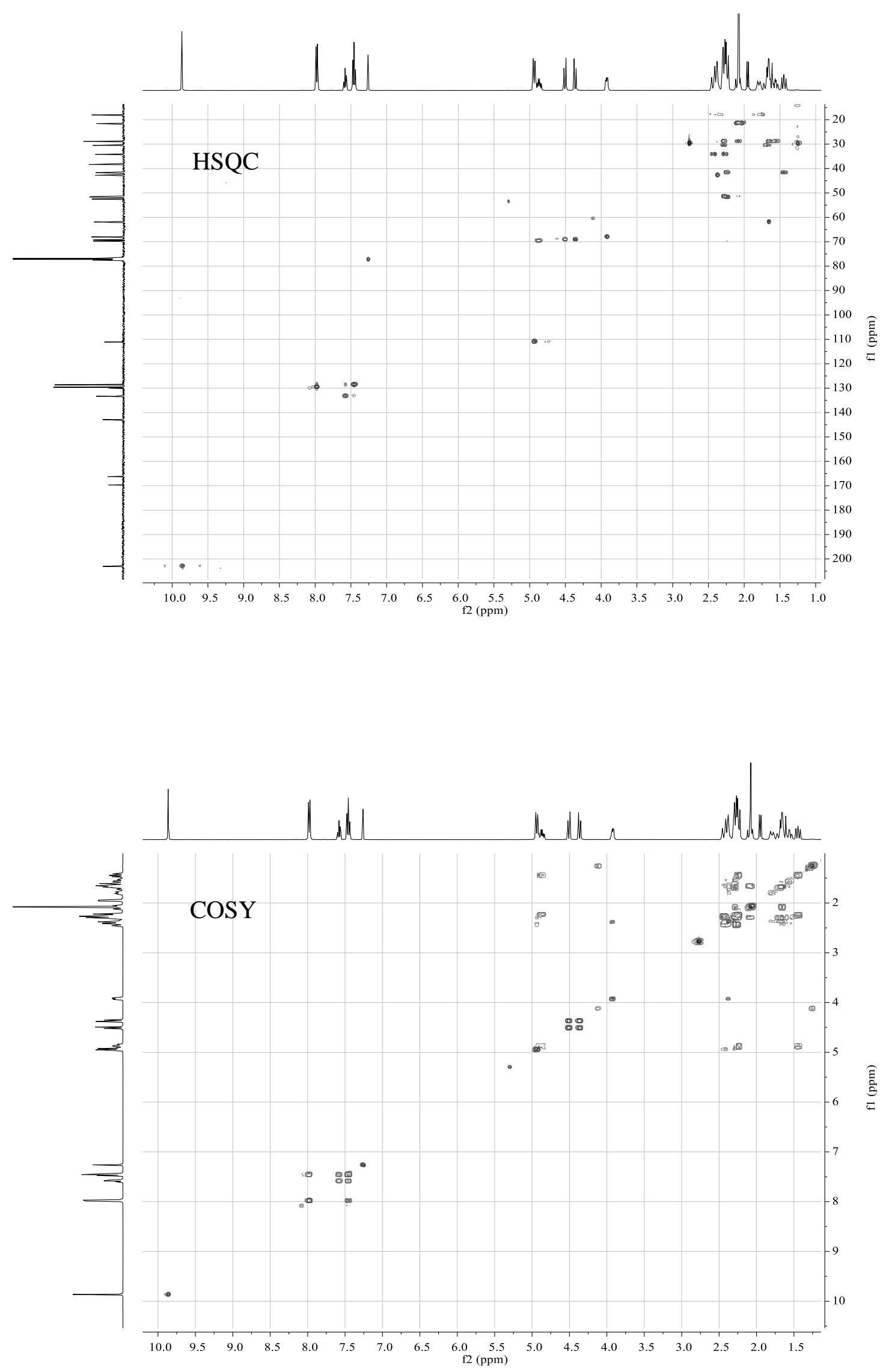

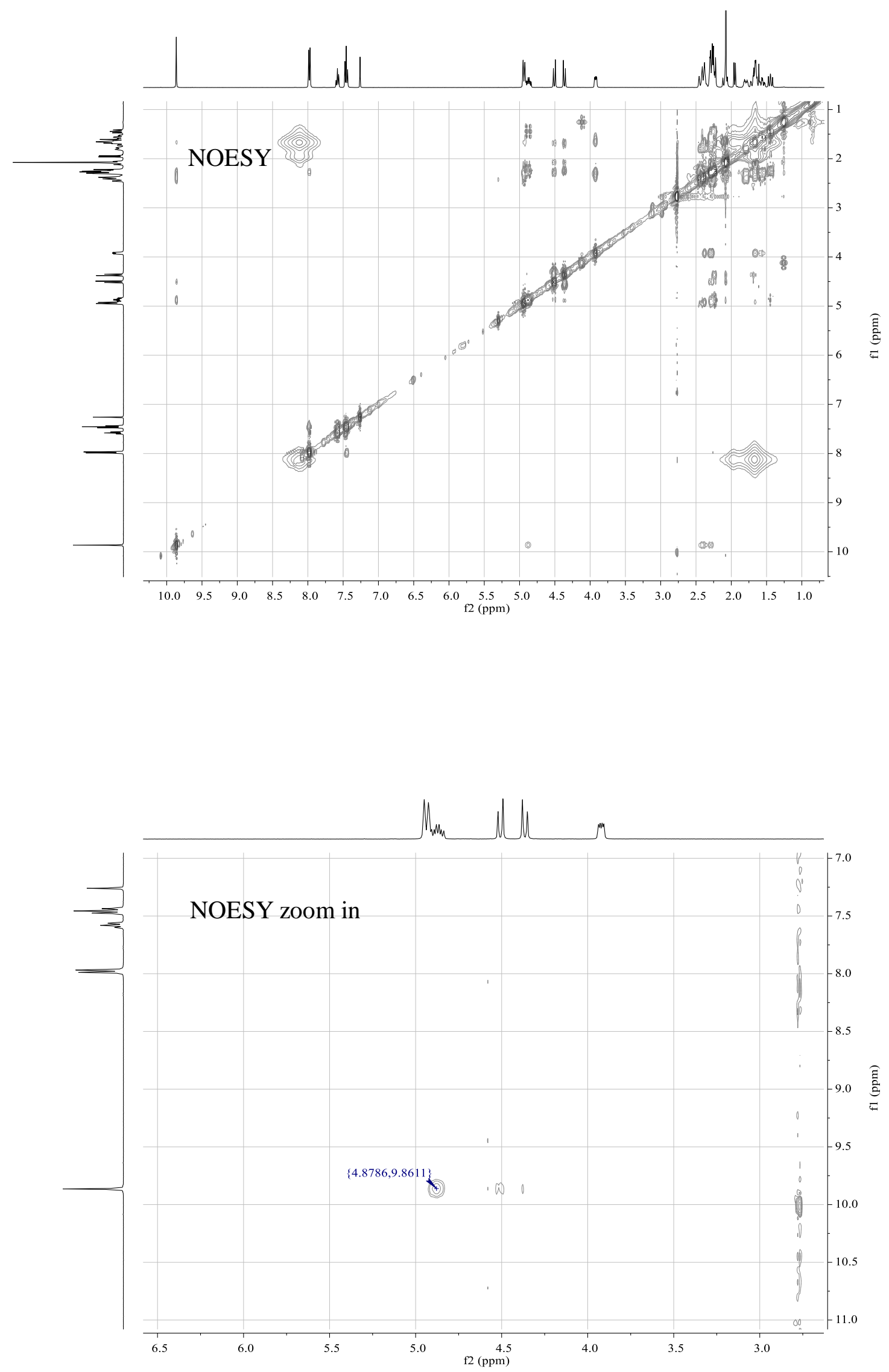


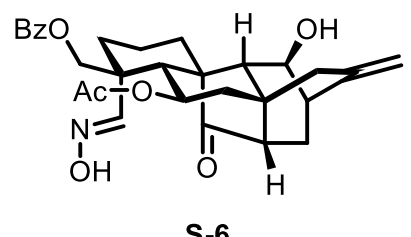

S-6

${ }^{1} \mathrm{H}$ NMR spectrum $\left(400 \mathrm{MHz}, \mathrm{CDCl}_{3}\right)$

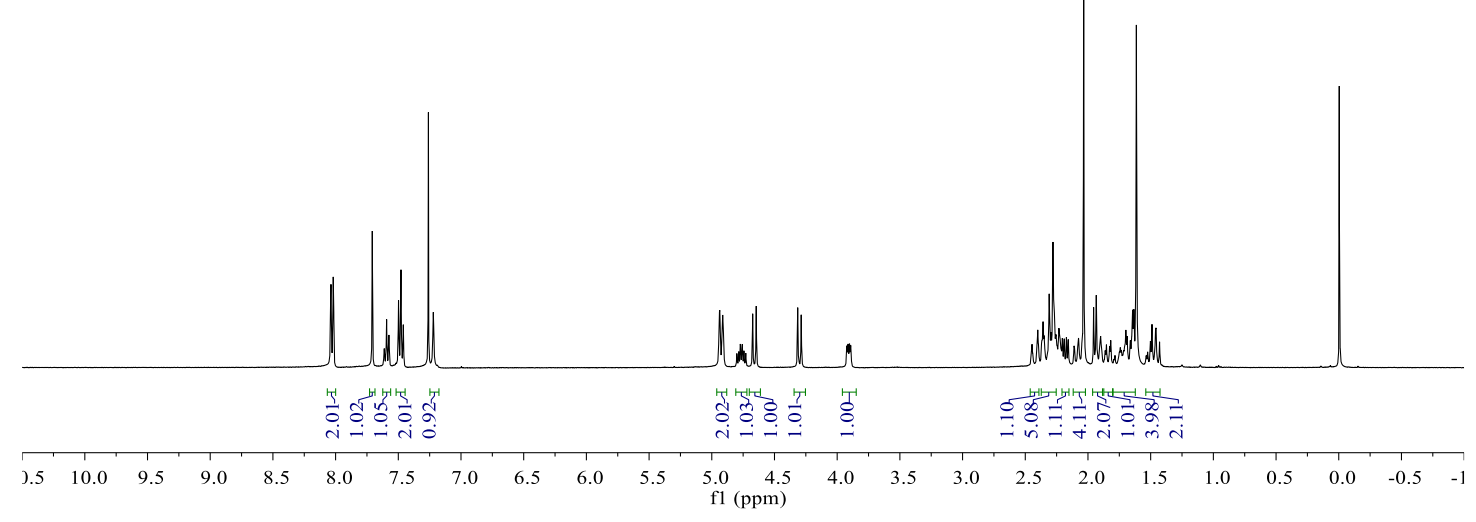

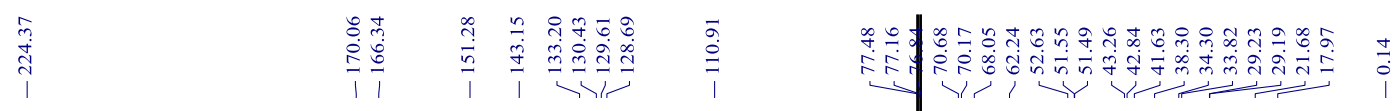

${ }^{13} \mathrm{C}$ NMR spectrum $\left(100 \mathrm{MHz}, \mathrm{CDCl}_{3}\right)$

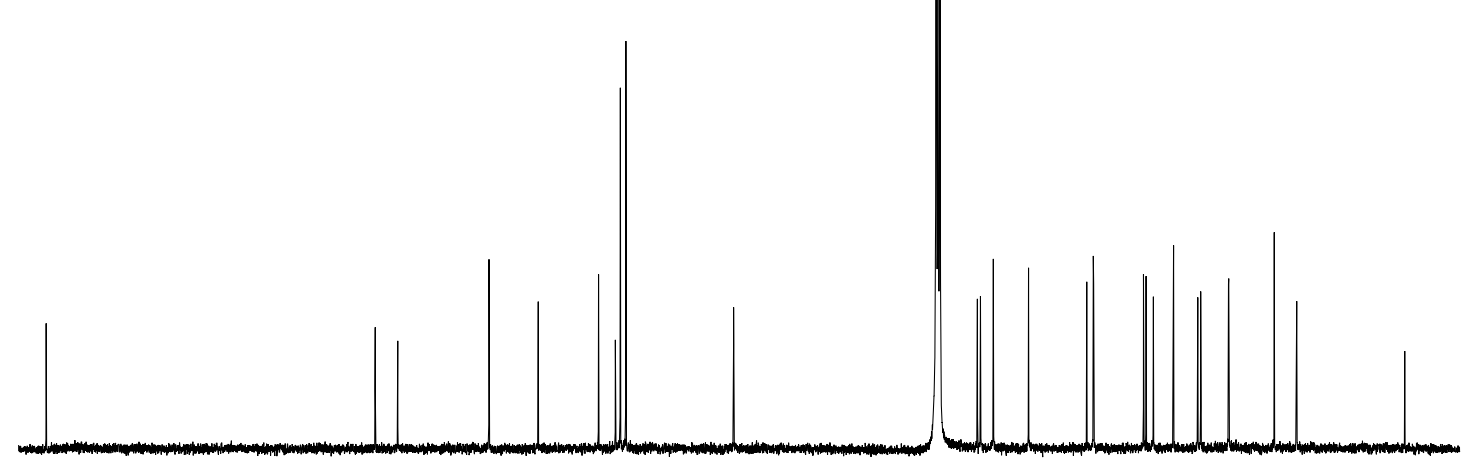

$\begin{array}{lllllllllllllllllllllll}220 & 210 & 200 & 190 & 180 & 170 & 160 & 150 & 140 & 130 & 120 & 110 & 100 & 90 & 80 & 70 & 60 & 50 & 40 & 30 & 20 & 10 & 0\end{array}$ 


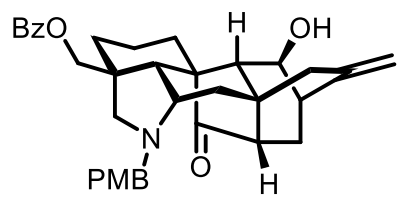

29

${ }^{1} \mathrm{H}$ NMR spectrum $\left(400 \mathrm{MHz}, \mathrm{CDCl}_{3}\right)$

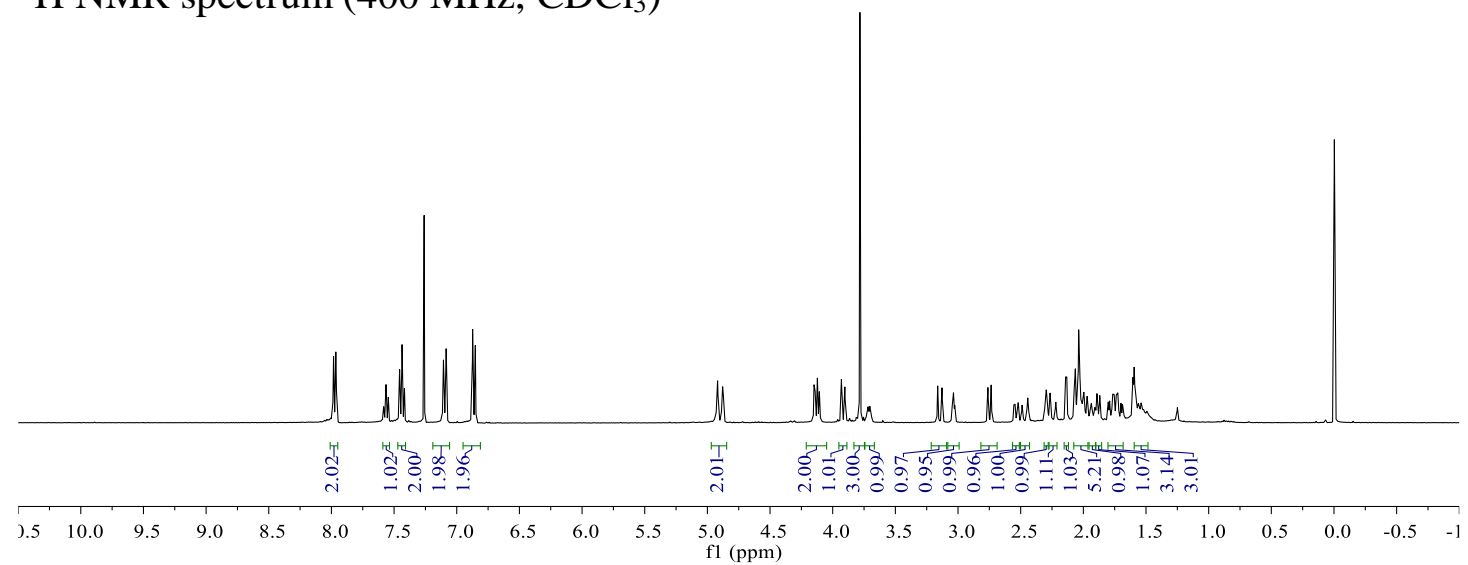

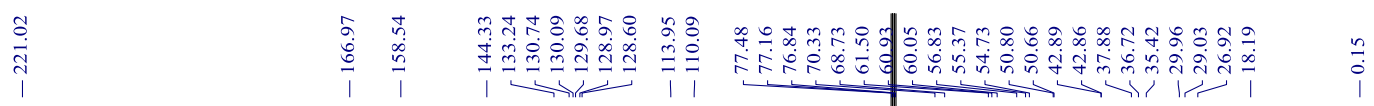

${ }^{13} \mathrm{C}$ NMR spectrum $\left(100 \mathrm{MHz}, \mathrm{CDCl}_{3}\right)$

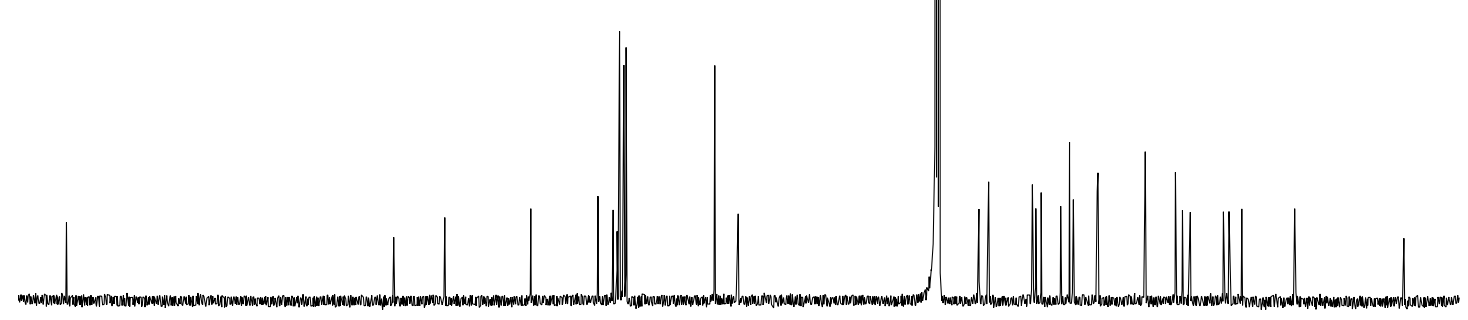

$\begin{array}{lllllllllllllllllllllllll}220 & 210 & 200 & 190 & 180 & 170 & 160 & 150 & 140 & 130 & 120 & 110 & 100 & 90 & 80 & 70 & 60 & 50 & 40 & 30 & 20 & 10 & 0\end{array}$ 


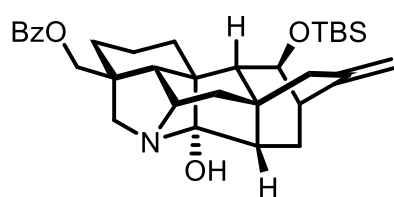

30

${ }^{1} \mathrm{H}$ NMR spectrum $\left(400 \mathrm{MHz}, \mathrm{CDCl}_{3}\right)$
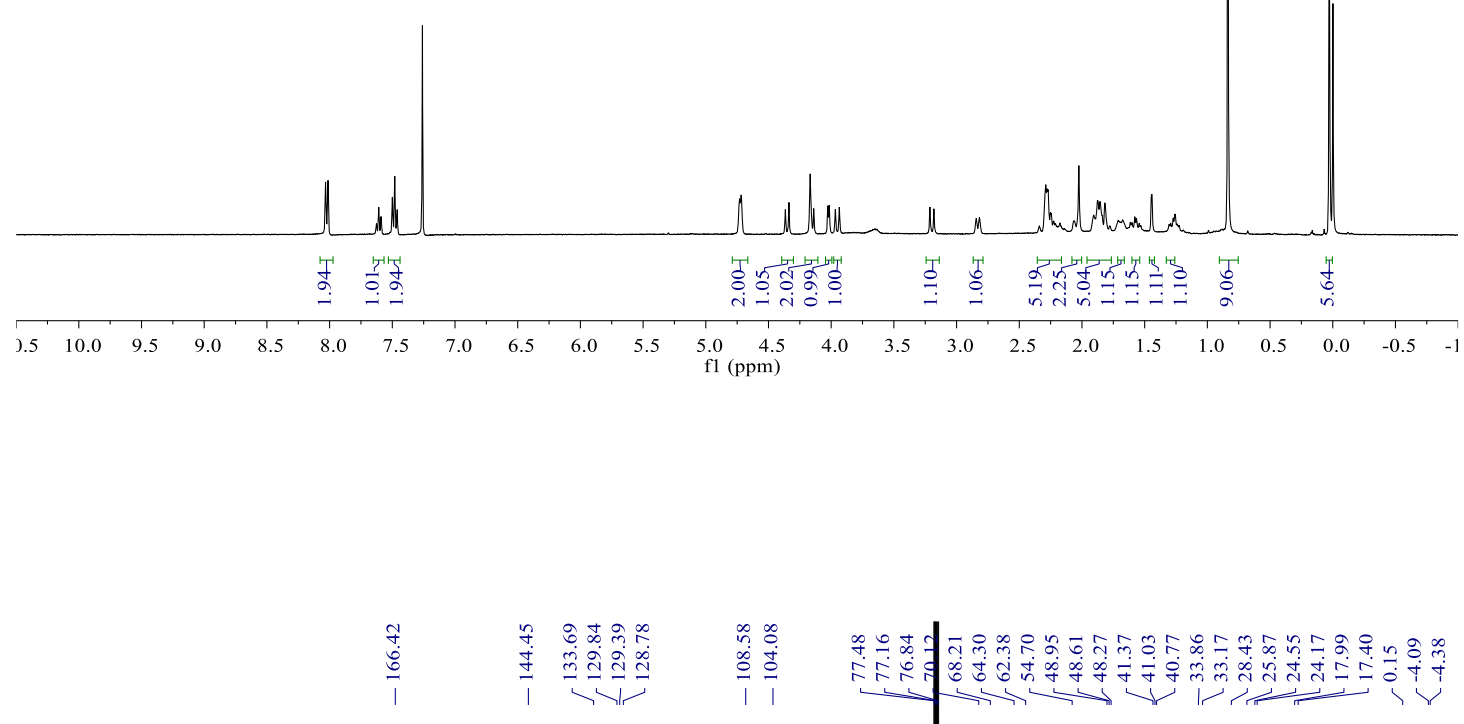

${ }^{13} \mathrm{C}$ NMR spectrum $\left(100 \mathrm{MHz}, \mathrm{CDCl}_{3}\right)$

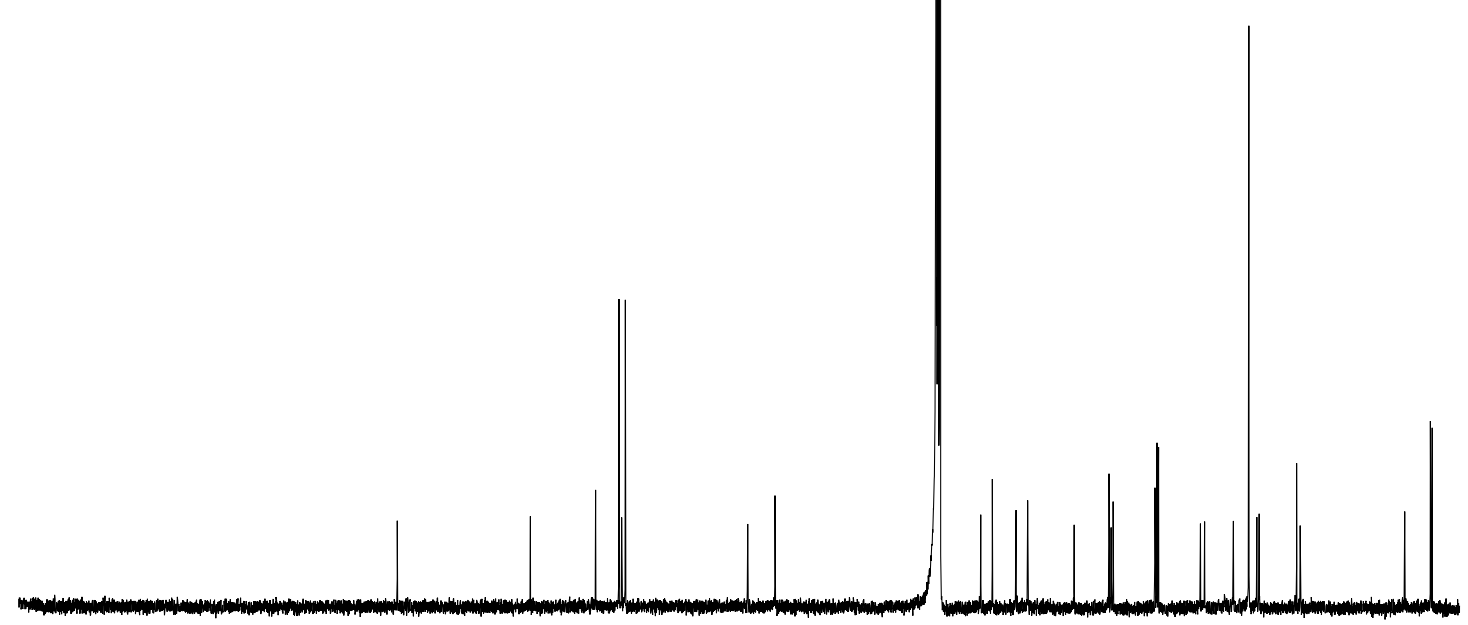

$\begin{array}{lllllllllllllllllllllllllllllllllll}220 & 210 & 200 & 190 & 180 & 170 & 160 & 150 & 140 & 130 & 120 & 110 & 100 & 90 & 80 & 70 & 60 & 50 & 40 & 30 & 20 & 10 & 0\end{array}$ 

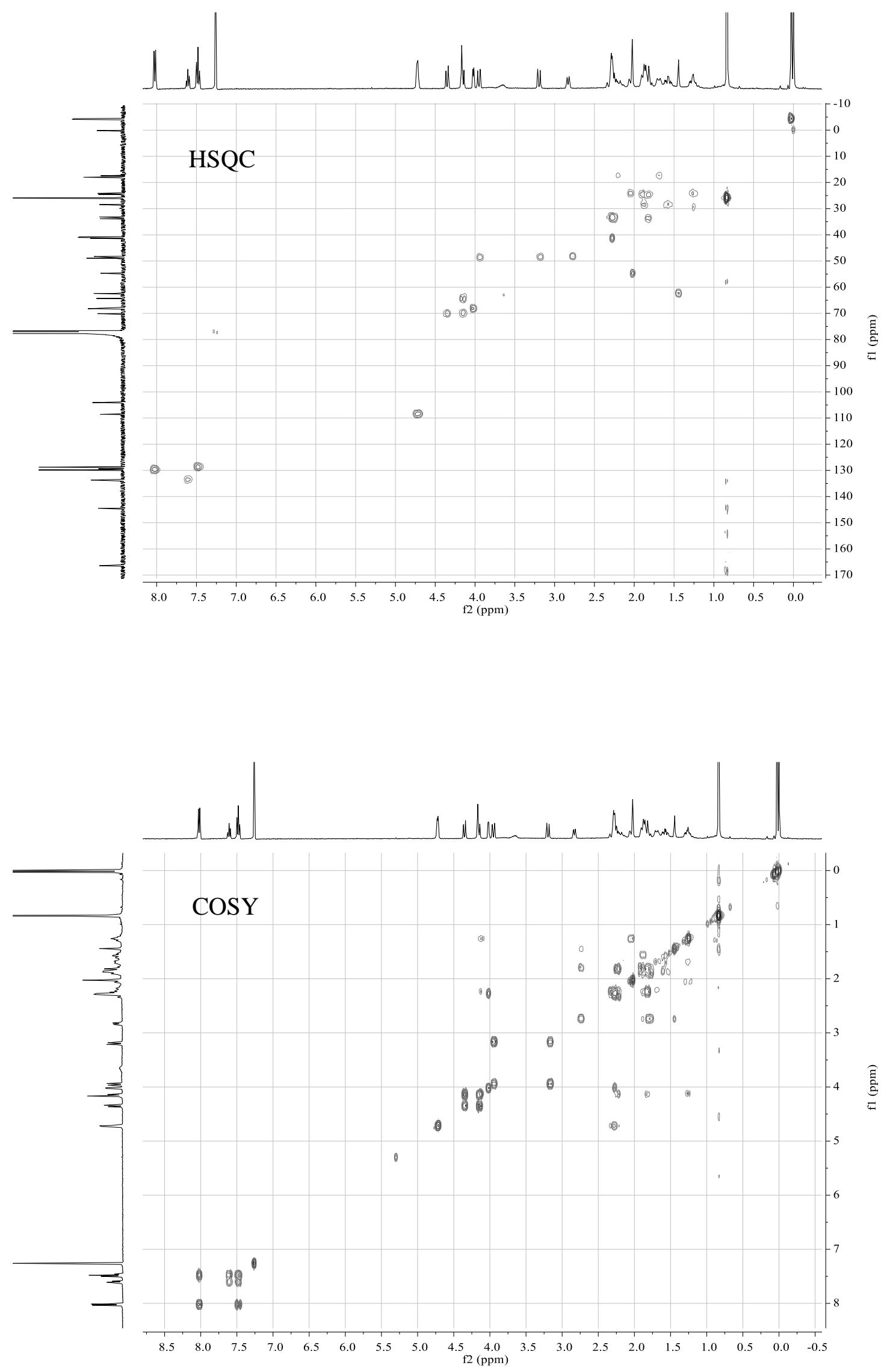


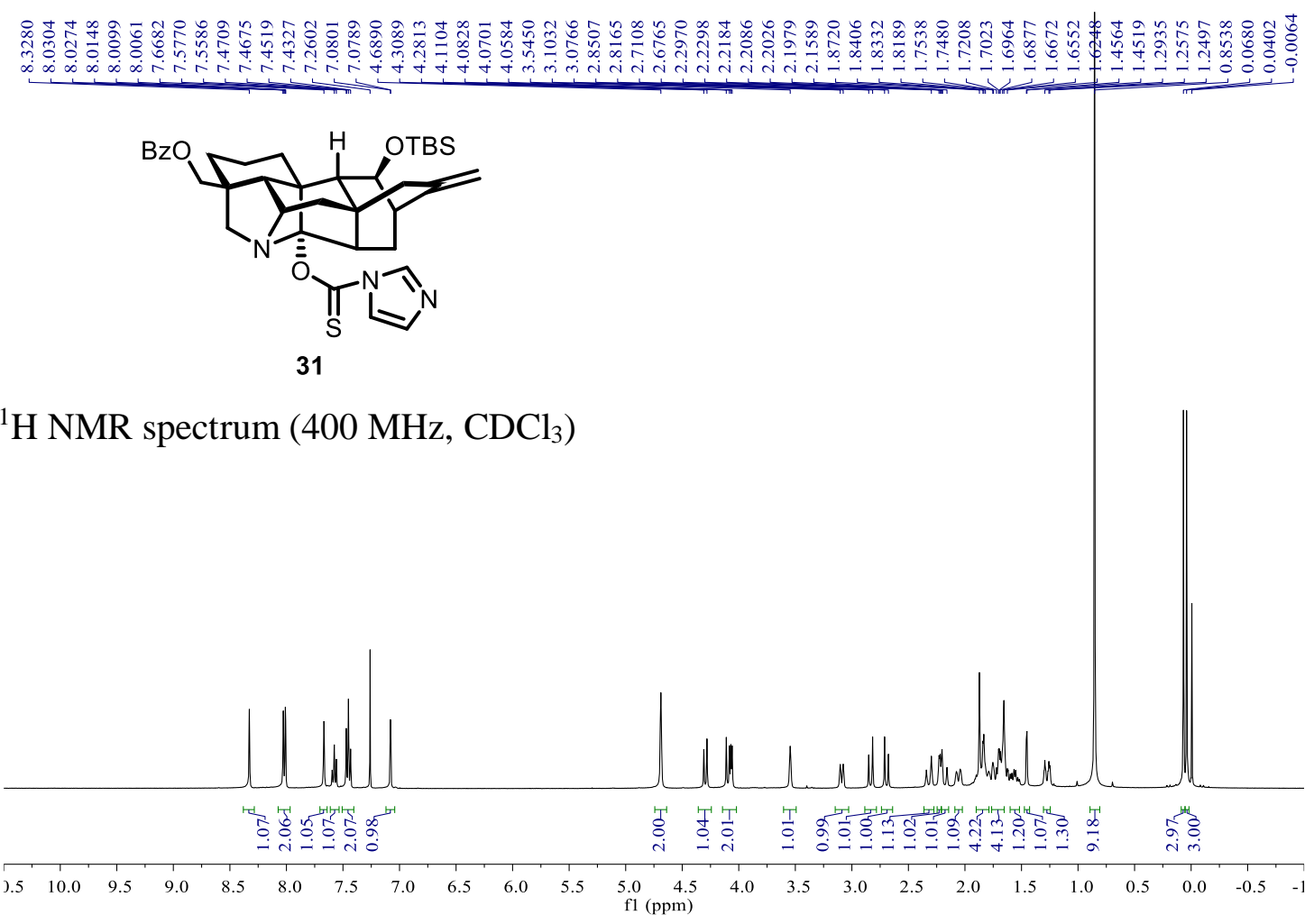

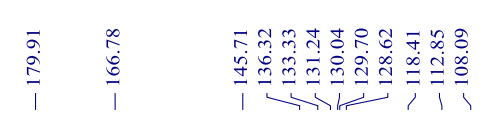

${ }^{13} \mathrm{C}$ NMR spectrum $\left(100 \mathrm{MHz}, \mathrm{CDCl}_{3}\right)$

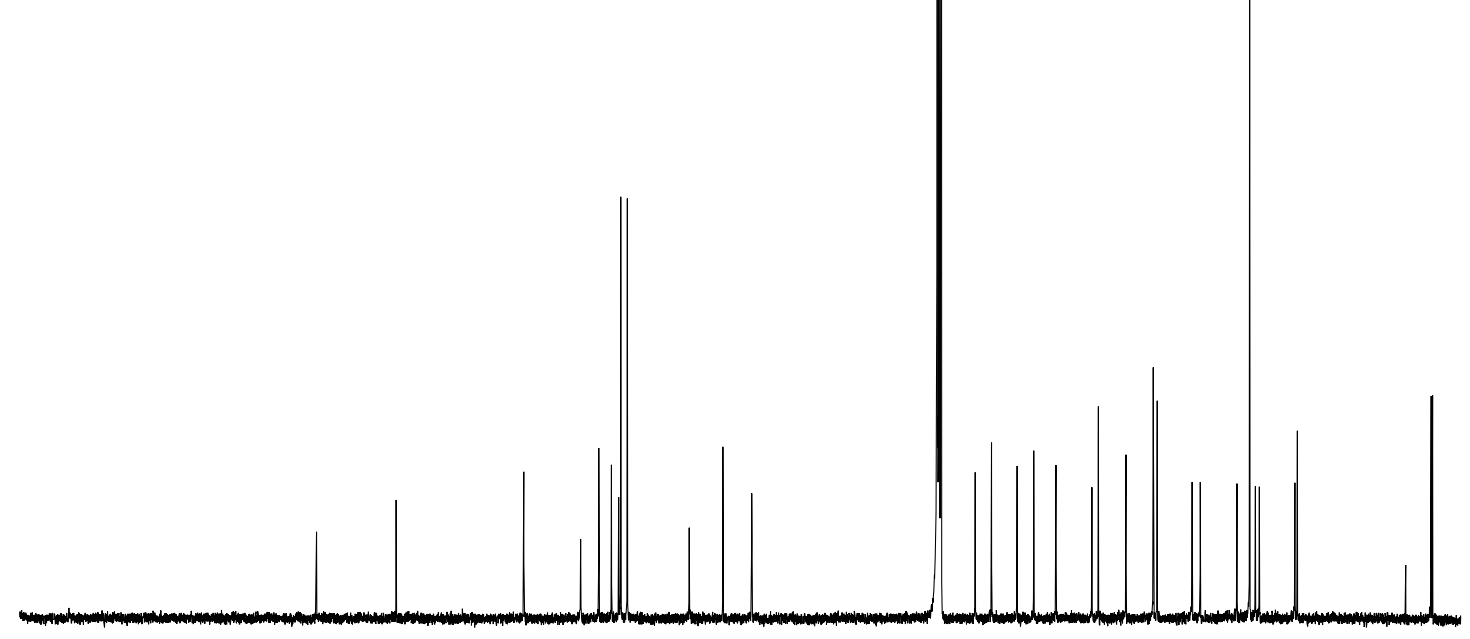

$\begin{array}{lllllllllllllllllllllllllllllll}220 & 210 & 200 & 190 & 180 & 170 & 160 & 150 & 140 & 130 & 120 & 110 & 100 & 90 & 80 & 70 & 60 & 50 & 40 & 30 & 20 & 10 & 0\end{array}$ 


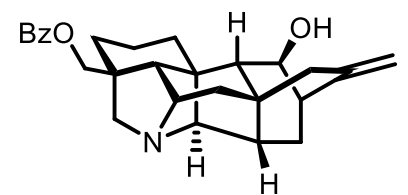

(+)-18-benzoyldavisinol (8)

${ }^{1} \mathrm{H}$ NMR spectrum $\left(400 \mathrm{MHz}, \mathrm{CDCl}_{3}\right)$

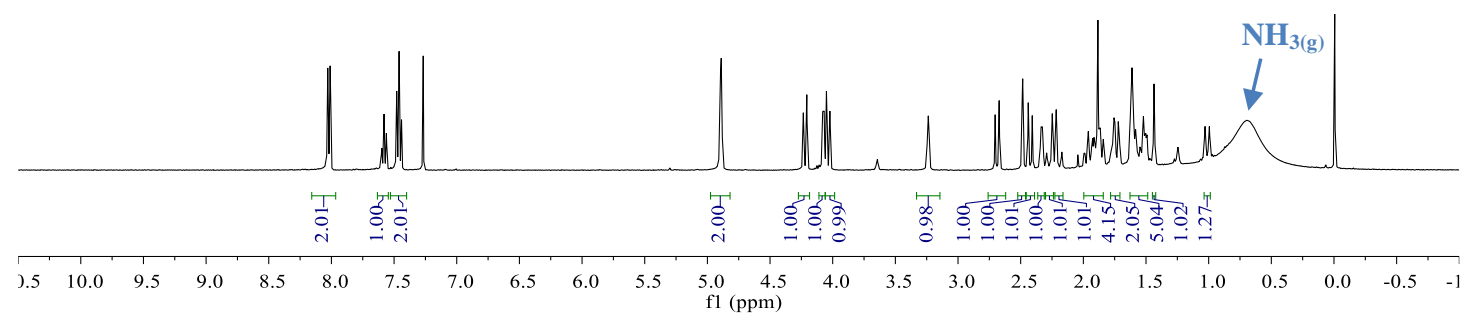

${ }^{13} \mathrm{C}$ NMR spectrum $\left(100 \mathrm{MHz}, \mathrm{CDCl}_{3}\right)$

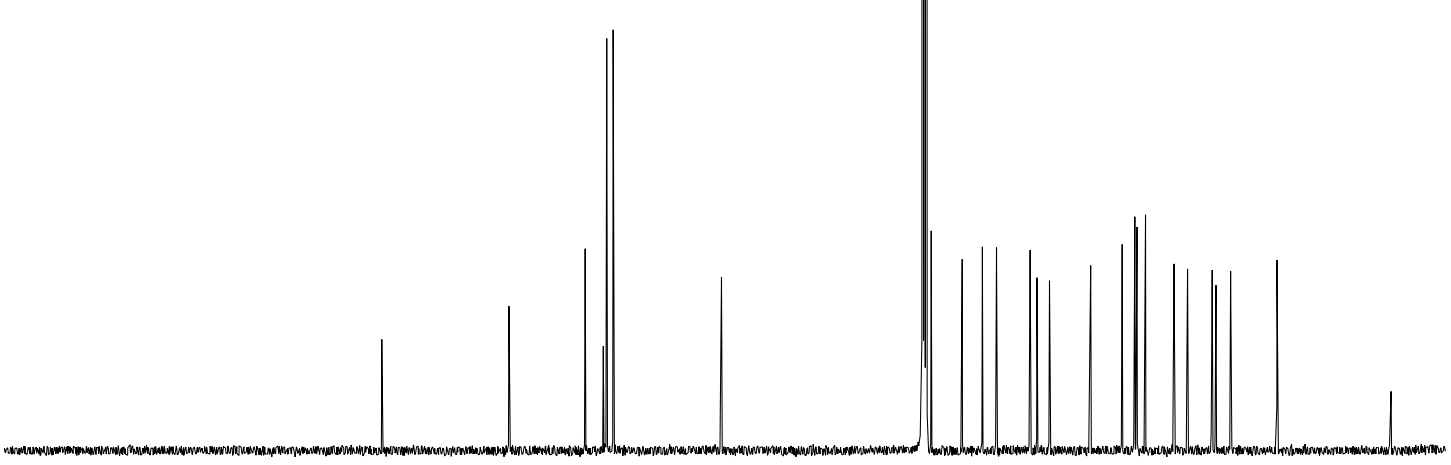

$\begin{array}{lllllllllllllllllllllllll}220 & 210 & 200 & 190 & 180 & 170 & 160 & 150 & 140 & 130 & 120 & 110 & 100 & 90 & 80 & 70 & 60 & 50 & 40 & 30 & 20 & 10 & 0\end{array}$ 

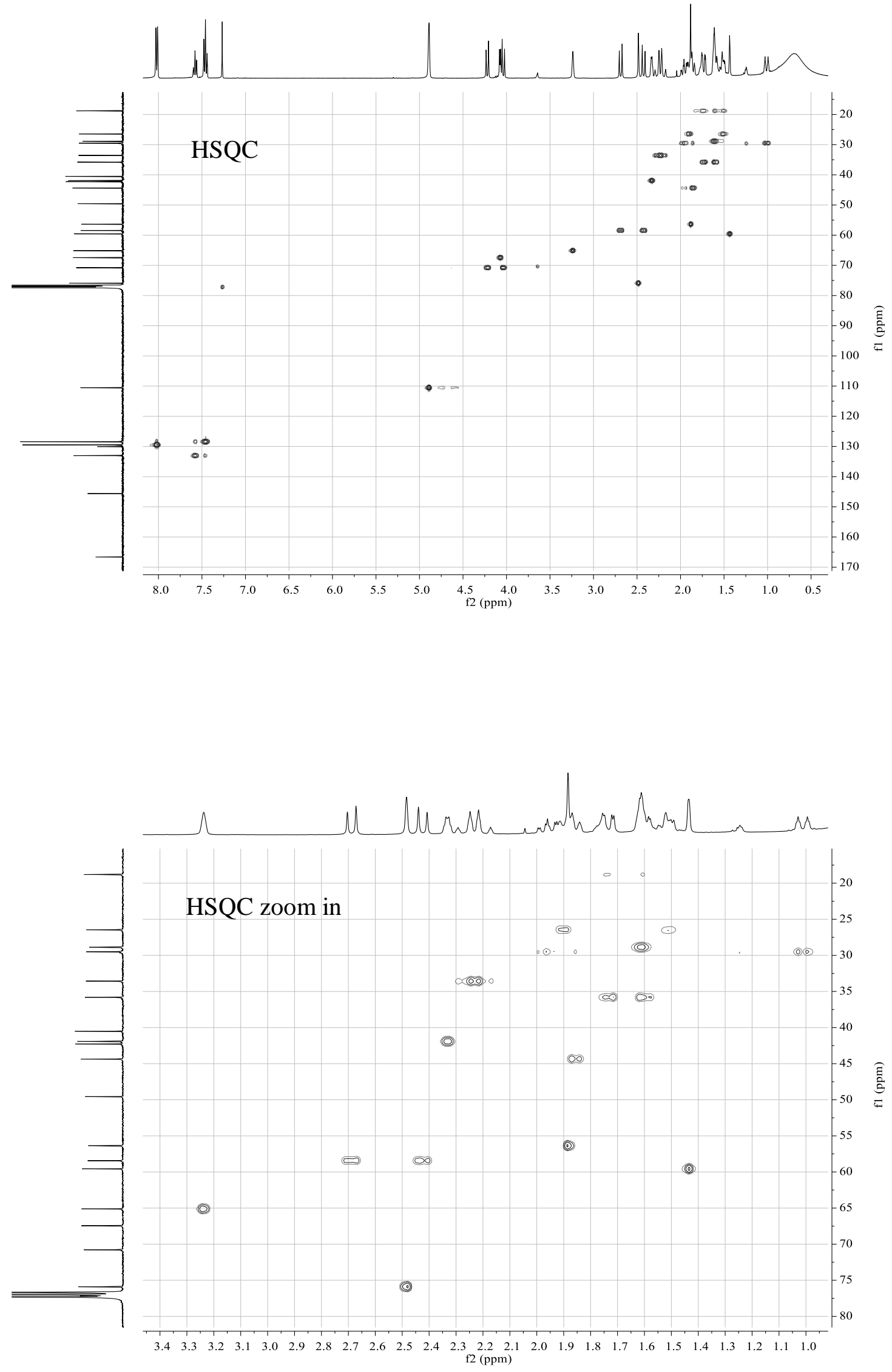

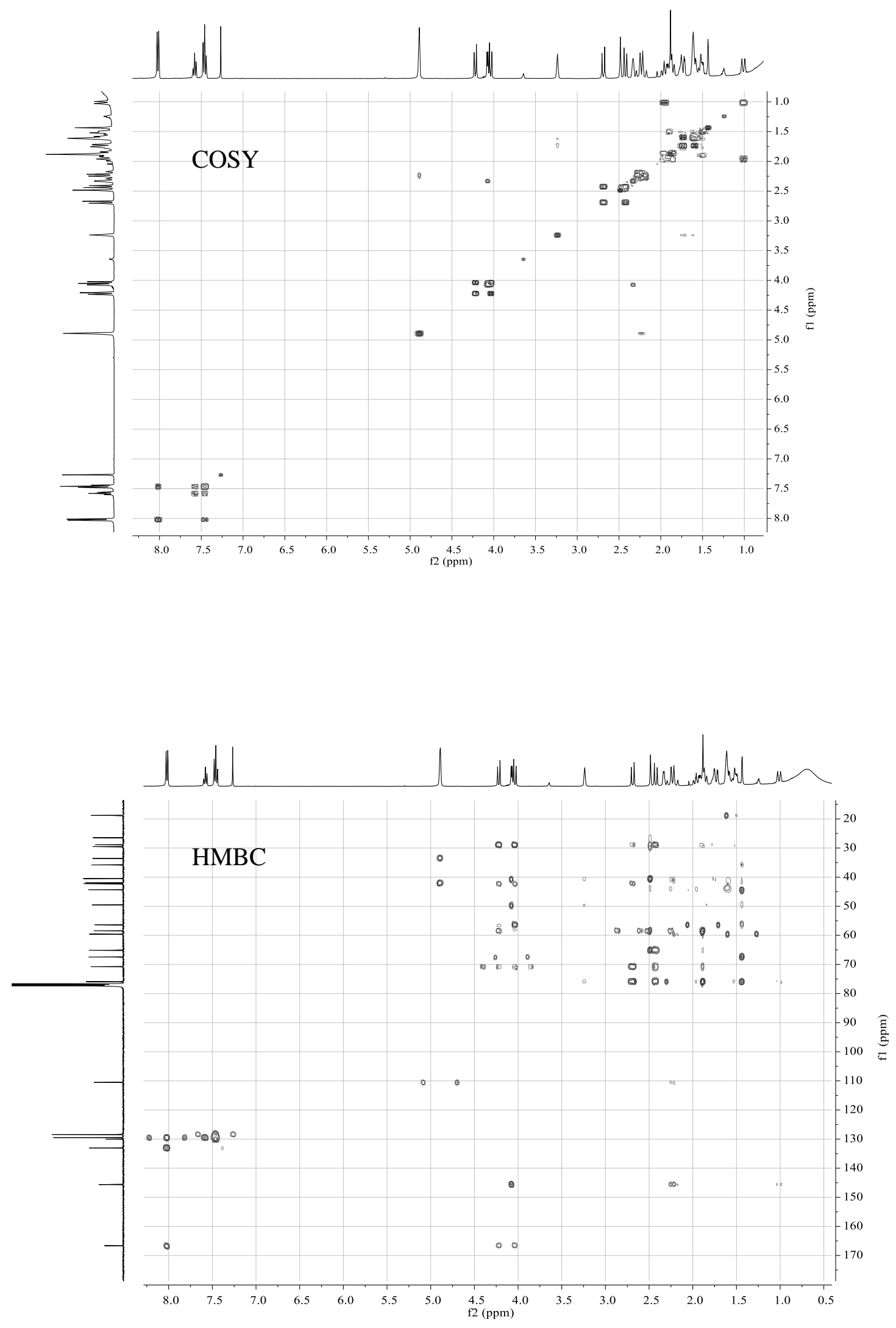


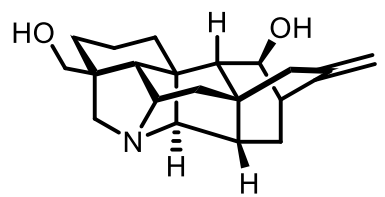

(+)-davisinol (7)

${ }^{1} \mathrm{H}$ NMR spectrum $\left(400 \mathrm{MHz}, \mathrm{CDCl}_{3}\right)$

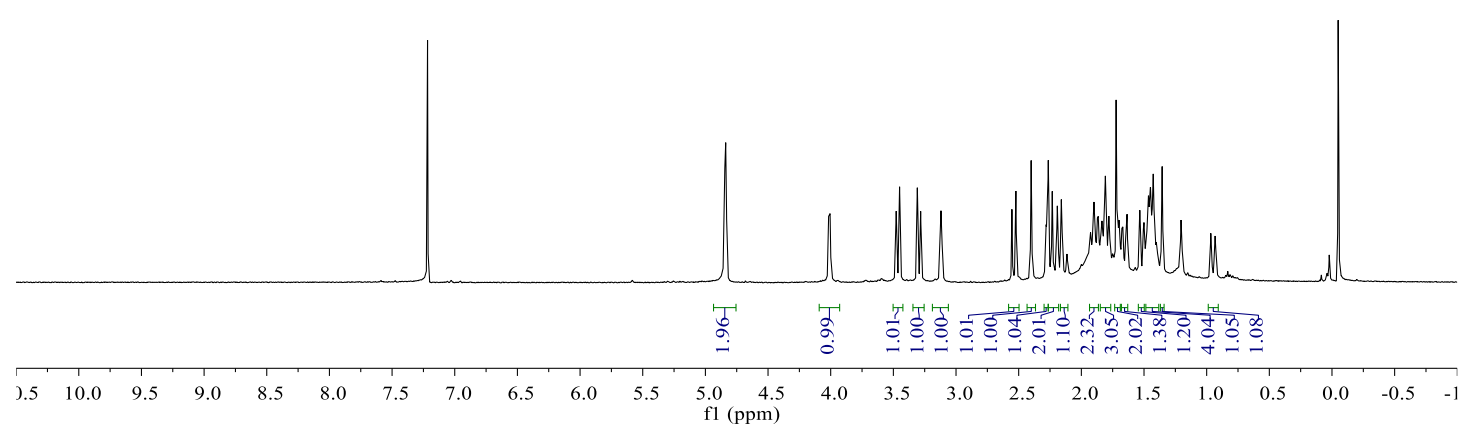

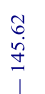

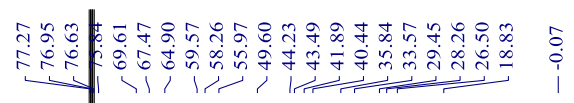

${ }^{13} \mathrm{C}$ NMR spectrum $\left(100 \mathrm{MHz}, \mathrm{CDCl}_{3}\right)$

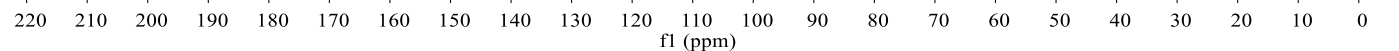



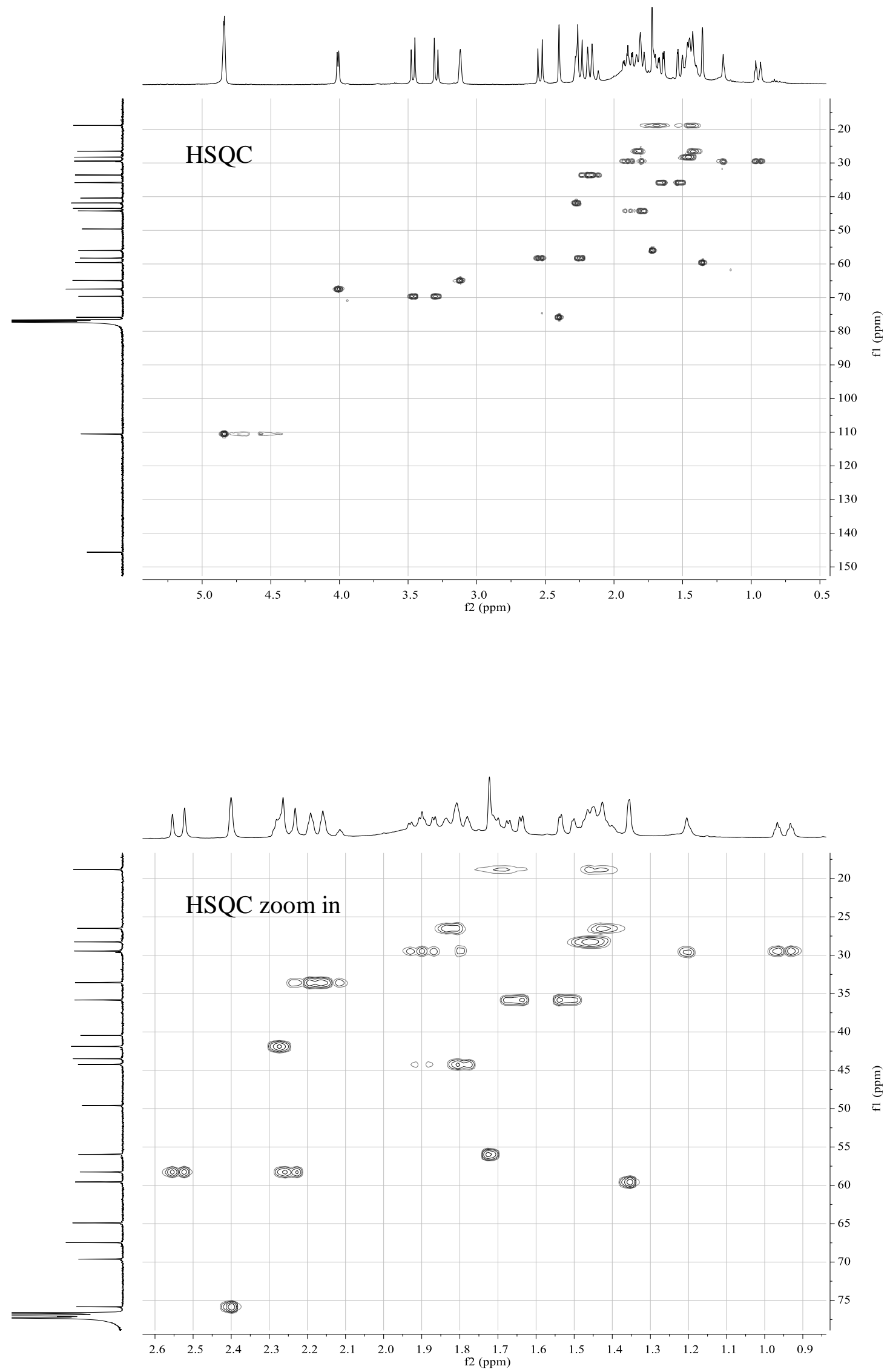

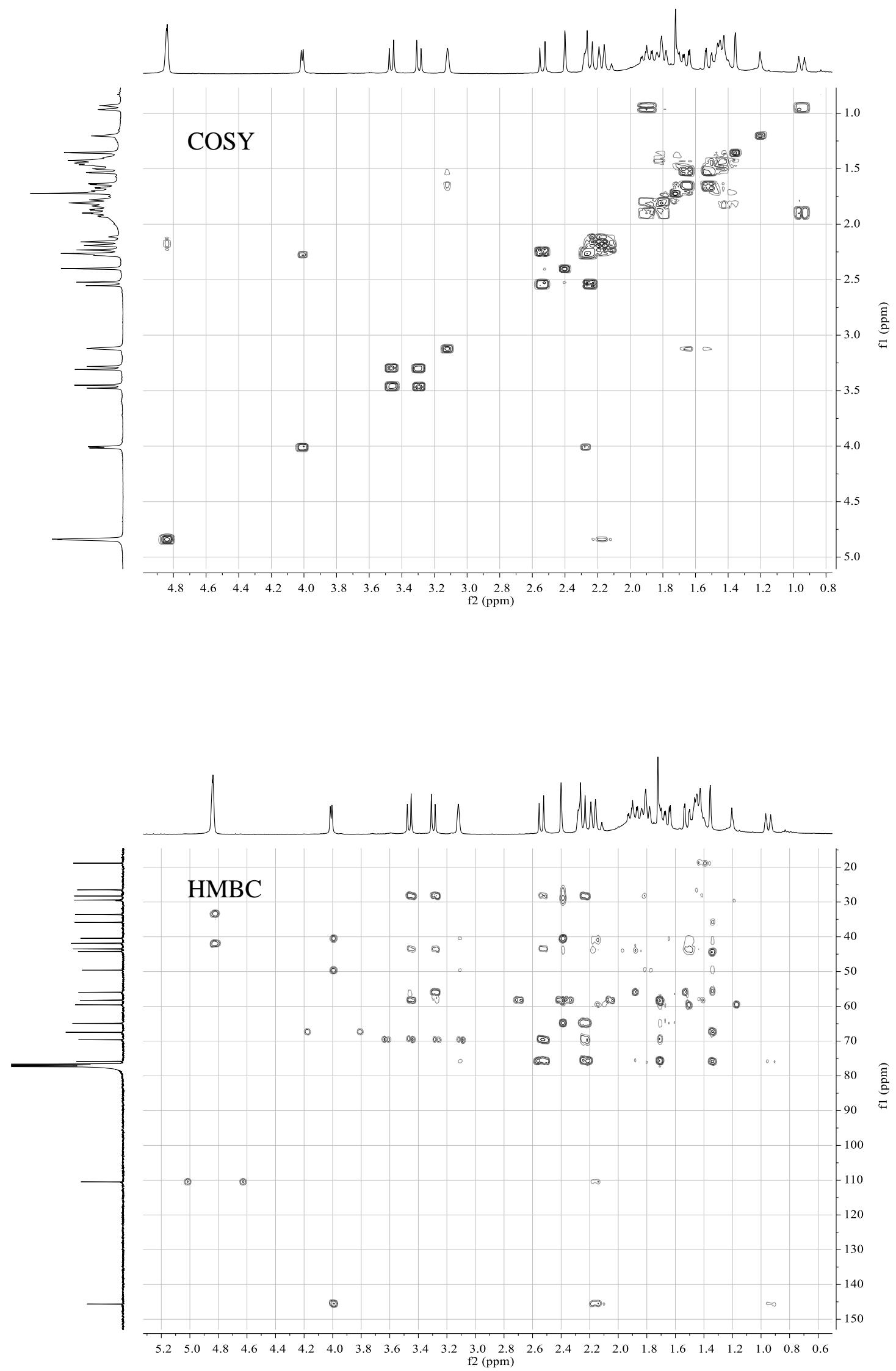


\section{VI) HPLC Chromatographs}

(1) Daicel Chiralpak IB $(0.46 \mathrm{~cm} \times 25 \mathrm{~cm}), n$-hexane/2-propanol $=99 / 1, \mathrm{v}=0.5$ $\mathrm{mL} \cdot \min ^{-1}, \lambda=210 \mathrm{~nm}$.

$\mathrm{mV}$

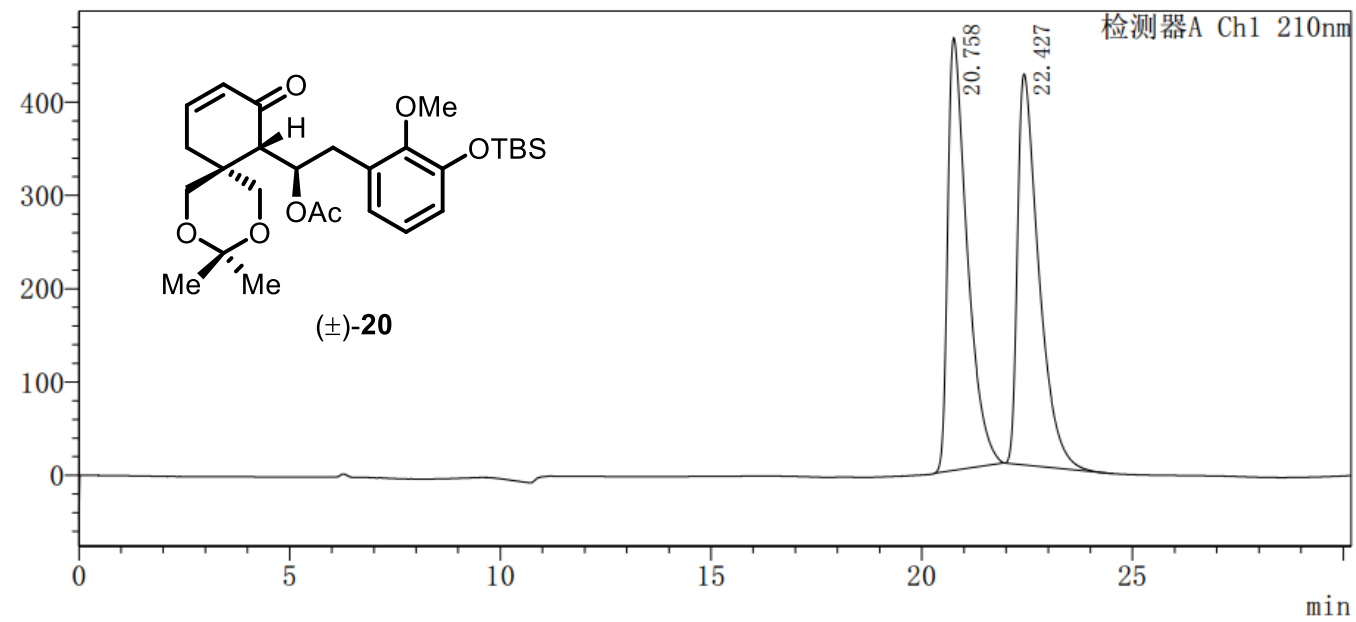

〈峰表〉

检测器A Ch1 210nm

\begin{tabular}{|r|r|c|r|r|r|r|r|}
\hline 峰号 & 保留时间 & 面积 & 高度 & 浓度 & 浓度单位 & 标记 & 化合物名 \\
\hline 1 & 20.758 & 14878689 & 463385 & 50.339 & & $\mathrm{M}$ & \\
\hline 2 & 22.427 & 14678031 & 418978 & 49.661 & & $\mathrm{M}$ & \\
\hline 总计 & & 29556720 & 882363 & & & & \\
\hline
\end{tabular}

$\mathrm{mV}$

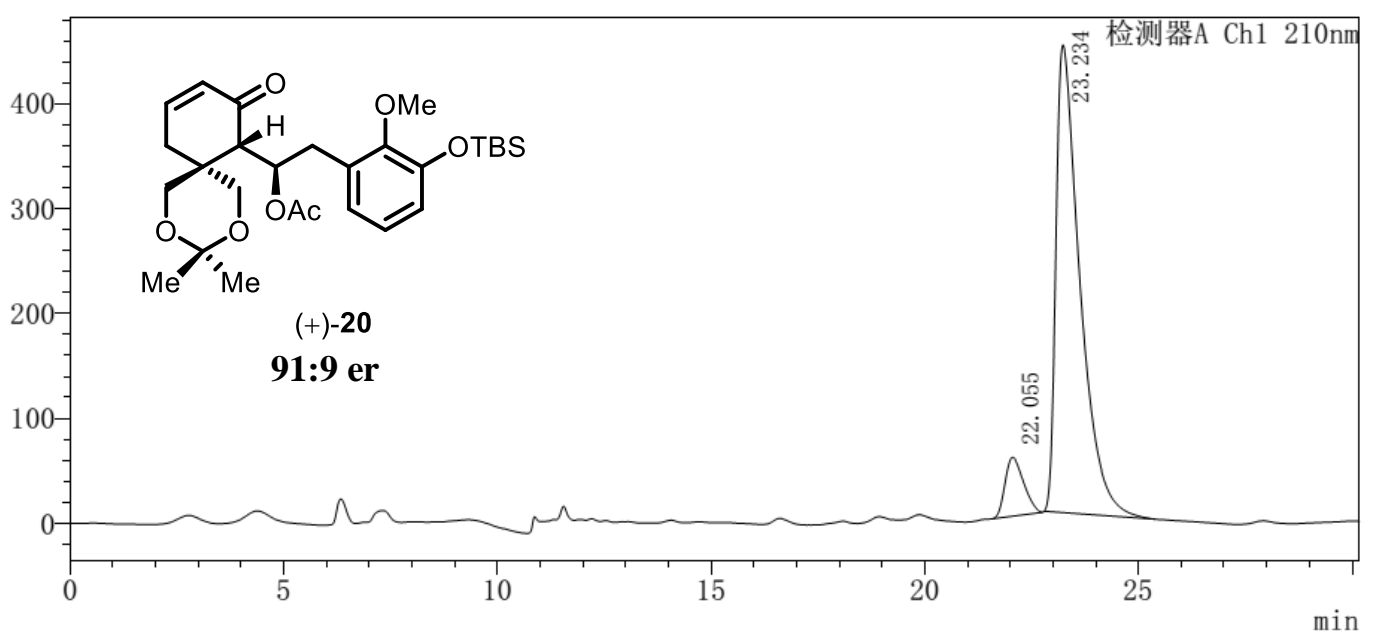

〈峰表〉

检测器A Ch1 210nm

\begin{tabular}{|r|r|r|r|r|r|r|r|}
\hline 峰号 & 保留时间 & 面积 & 高度 & 浓度 & 浓度单位 & 标记 & 化合物名 \\
\hline 1 & 22.055 & 1706836 & 56102 & 8.984 & & $\mathrm{M}$ & \\
\hline 2 & 23.234 & 17291492 & 445993 & 91.016 & & $\mathrm{M}$ & \\
\hline 总计 & & 18998328 & 502095 & & & & \\
\hline
\end{tabular}


(2) Daicel Chiralpak AD-H $(0.46 \mathrm{~cm} \times 25 \mathrm{~cm}), n$-hexane/2-propanol $=90 / 10, \mathrm{v}=1.0$ $\mathrm{mL} \cdot \min ^{-1}, \lambda=210 \mathrm{~nm}$.

$\mathrm{mV}$

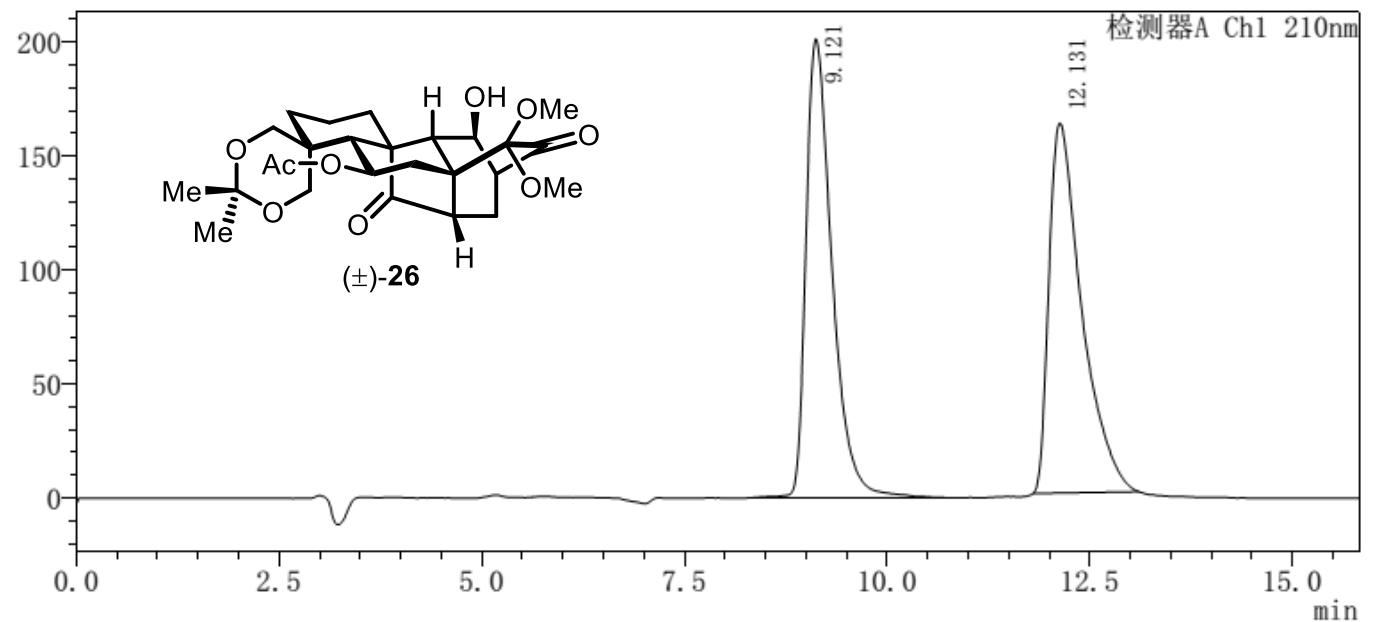

〈峰表〉

检测器A Ch1 210nm

峰号 保留时间

\begin{tabular}{|r|r|l|l|l|l|r|r|}
\hline 峰号 & 保留时间 & 面积 & 高度 & 浓度 & 浓度单位 & 标记 & 化合物名 \\
\hline 1 & 9.121 & 4502902 & 201189 & 49.722 & & $\mathrm{M}$ & \\
\hline 2 & 12.131 & 4553235 & 162185 & 50.278 & & $\mathrm{M}$ & \\
\hline 总计 & & 9056136 & 363374 & & & &
\end{tabular}

$\mathrm{mV}$

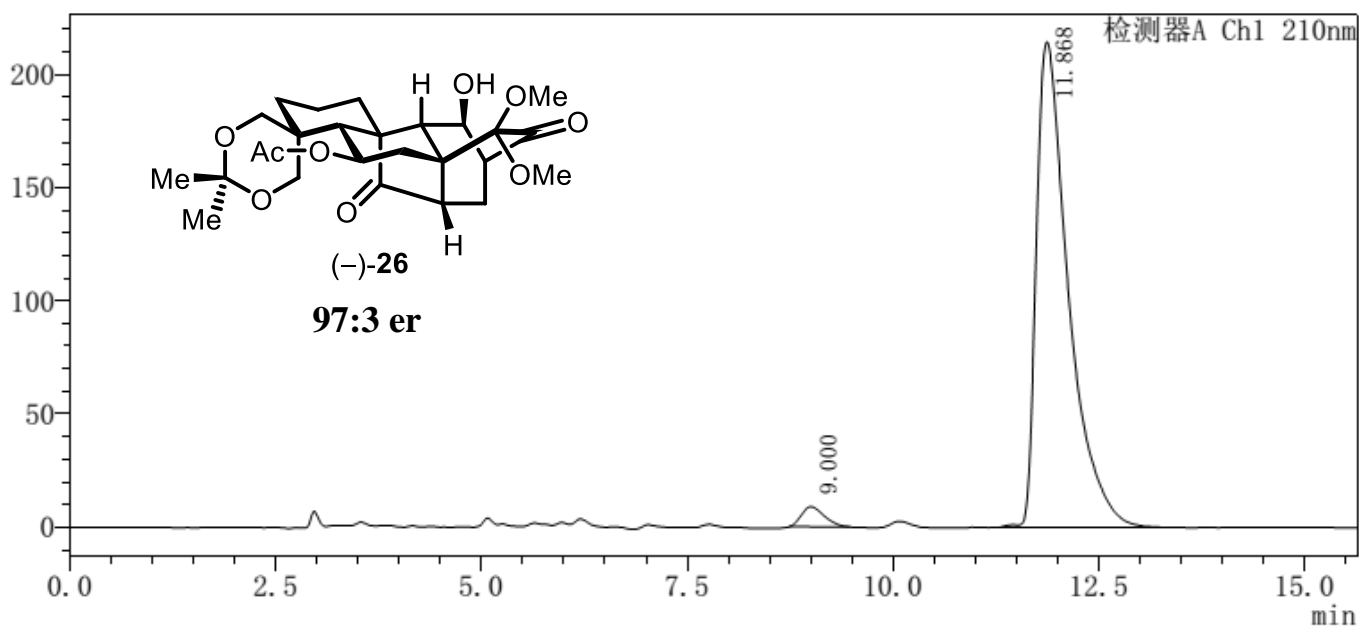

〈峰表〉

检测器A Ch1 210nm

\begin{tabular}{|r|r|r|r|r|r|r|r|}
\hline 峰号 & 保留时间 & \multicolumn{1}{|c|}{ 面积 } & 高度 & 浓度 & 浓度单位 & 标记 & 化合物名 \\
\hline 1 & 9.000 & 170656 & 8859 & 2.844 & & $\mathrm{M}$ & \\
\hline 2 & 11.868 & 5830537 & 214551 & 97.156 & & $\mathrm{M}$ & \\
\hline 总计 & & 6001192 & 223410 & & & & \\
\hline
\end{tabular}

